\title{
Langevin diffusion of heavy quarks in non-conformal holographic backgrounds
}

\author{
Umut Gürsoy, ${ }^{a}$ Elias Kiritsis, ${ }^{b, d}$ Liuba Mazzanti $^{c}$ and Francesco Nitti ${ }^{d}$ \\ ${ }^{a}$ Institute for Theoretical Physics, Utrecht University, \\ Leuvenlaan 4, 3584 CE Utrecht, The Netherlands \\ ${ }^{b}$ Crete Center for Theoretical Physics, Department of Physics, University of Crete, \\ 71003 Heraklion, Greece \\ ${ }^{c}$ Departamento de Física de Partículas, Universidade de Santiago de Compostela and \\ Instituto Galego de Física de Altas Enerxías (IGFAE), \\ E-15782, Santiago de Compostela, Spain \\ ${ }^{d} A P C$, UMR du CNRS 7164, Université Paris 7, \\ Bâtiment Condorcet, F-75205, Paris Cedex 13, France \\ E-mail: Umut.Gursoy@cern.ch, kiritsis@physics.uoc.gr, \\ mazzanti@fpaxp1.usc.es, nitti@apc.univ-paris7.fr
}

ABSTRACT: The Langevin diffusion process of a relativistic heavy quark in a non-conformal holographic setup is analyzed. The bulk geometry is a general, five-dimensional asymptotically AdS black hole. The heavy quark is described by a trailing string attached to a flavor brane, moving at constant velocity. From the equations describing linearized fluctuations of the string world-sheet, the correlation functions defining a generalized Langevin process are constructed via the AdS/CFT prescription. In the local limit, analytic expressions for the Langevin diffusion and friction coefficients are obtained in terms of the bulk string metric. Modified Einstein relations between these quantities are also derived. The spectral densities associated to the Langevin correlators are analyzed, and simple analytic expressions are obtained in the small and large frequency limits. Finally, a numerical analysis of the jet-quenching parameter, and a comparison to RHIC phenomenology are performed in the case of Improved Holographic QCD. It is shown that the jet-quenching parameter is not enough to describe energy loss of very energetic charm quarks and the full Langevin correlators are needed.

KEYwORDS: Gauge-gravity correspondence, Black Holes, QCD

ARXIV EPRINT: 1006.3261 


\section{Contents}

1 Introduction and results $\quad 2$

2 Langevin equation for a relativistic heavy quark $\quad 8$

2.1 The Langevin equation in the boundary theory 8

$\begin{array}{lll}2.2 & \text { The Langevin equation in the gravity dual picture } & 12\end{array}$

3 5D non-conformal backgrounds for Langevin holography 14

3.1 5D Einstein-Dilaton black holes 15

$\begin{array}{lll}3.2 & \text { Classical trailing string and the drag force } & 16\end{array}$

$\begin{array}{lll}3.3 & \text { Fluctuations of the trailing string } & 18\end{array}$

4 Holographic computation of Langevin correlators 20

4.1 The Green's functions 20

4.2 The membrane paradigm 24

4.2.1 A differential equation for spectral densities 25

4.3 Universal results for the spectral densities 26

$\begin{array}{lll}4.4 & \text { The WKB approximation at large frequency } & 27\end{array}$

$5 \quad$ Langevin diffusion constants $\quad 29$

5.1 Diffusion constants via the retarded correlator 30

5.2 The jet-quenching parameter 31

5.3 The diffusion constants via the membrane paradigm 32

5.4 A universal inequality: $\boldsymbol{\kappa}_{\|} \geq \boldsymbol{\kappa}_{\perp} \quad 32$

5.5 A generalized Einstein relation 33

5.6 Special limits of the diffusion constants 34

$\begin{array}{lll}5.6 .1 & \text { Non-relativistic limit } & 35\end{array}$

5.6.2 Ultra-relativistic limit 35

5.7 Time scales and validity of the local approximation 36

6 Improved Holographic QCD and comparison with data 38

$\begin{array}{ll}6.1 \text { Correlators and spectral functions } & 40\end{array}$

6.2 The jet-quenching parameters 44

6.3 Comparison with heavy-ion collision observables 48

A Boundary terms and scheme dependence in the propagator 52

B Analytic calculation of the diffusion constants 54

C Details of the WKB approximation $\quad 56$

D Correlators in $\mathcal{N}=4 \quad 62$ 


\section{Introduction and results}

RHIC experiments of heavy-ion collisions and related data on the deconfined phase of QCD, [1-4] have provided a window for string theory techniques to meet the real world. The context is strong coupling dynamics near and above the deconfining transition in QCD. String theory via the AdS/CFT correspondence has provided a framework in order to understand strong coupling dynamics in the deconfined phase including the calculation of transport coefficients. Recent reviews on the progress in this direction are [5-9].

Observables of particular importance are associated to heavy quarks. Heavy quarks may be produced in the Quark-Gluon Plasma (QGP) of the RHIC fireball and are then travelling to the detectors while moving through the dense QGP. They can be tagged reasonably well and are therefore valuable probes of the dynamics in the plasma and in particular for the mechanism of energy loss.

A single heavy quark can be modeled in string theory by an open string. Its end-point is representing the heavy quark while the string is trailing behind as the quark moves. The large mass limit is important in order to neglect the non-trivial flavor dynamics associated with light quarks (although with improved techniques the light quarks may also eventually be addressed reliably in the holographic context). As quarks are associated with strings ending on flavor branes, a heavy quark ends on a brane that is stretching in the UV part of the bulk geometry. The motion of such a string, and the associated force acting on the quark from the thermal medium, have been studied in detail with several complementary methods, [10-13]. In the simplest setup, the UV endpoint of a fundamental string is forced to move with constant velocity $v$ along a spacial direction. The equations of motion for the full string are solved and the radial profile of the trailing string is found as it moves in a bulk black-hole background representing the deconfined heat bath. The energy absorbed by the string is calculated and the drag force of the string is obtained. The picture remains roughly valid, while details change when conformal invariance is broken, [14, 15].

An important improvement in this picture consists of the study of the stochastic nature of this system in analogy with the dynamics of heavy particles in a heat bath giving rise to Brownian motion. This involves a diffusive process, that was first considered in a holographic setting in [16], by using the Schwinger-Keldysh formalism adapted to AdS/CFT in [17].

Subsequently, a study of the (quantum) fluctuations of the trailing string, $[18,19]$ provided the information on the momentum broadening of a heavy quark as it moves in the plasma. The stochastic motion was formulated as a Langevin process, [20, 21] associated with the correlators of the fluctuations of the string.

Many heavy quarks in experiments are relativistic. Therefore it is necessary to study the associated relativistic Langevin evolution of the trailing string, a feat accomplished in the $\mathcal{N}=4$ case in [22]. The same type of Langevin process was studied in [23] for the case of an accelerating quark in the vacuum (rather than in a deconfined plasma), by analyzing the fluctuations of a trailing string in $\mathrm{AdS}$ with a non-uniformly moving endpoint.

On the experimental front, there have been several results from the RHIC experiments, [25]-[29]. The experimental signatures are currently summarized by the $e^{ \pm}$spectra 
that originate in the semileptonic decays of charmed and bottom hadrons. From these spectra a modification factor $R_{A A}^{e}$ and an elliptic flow coefficient $v_{2}^{e}$ are extracted. They capture the effects of the medium to the propagation of the heavy quarks. The data exhibit a substantial elliptic flow, up to $v_{2}^{e} \simeq 10 \%$, and a high- $p_{T}$ suppression down to $R_{A A}^{e} \simeq 0.25$. These values are comparable to light hadrons. Radiative energy loss models based on pQCD, [30] do not seem to explain well the experimental data, [31]. Elastic scattering energy loss plus non-perturbative interactions can on the other hand accommodate the data, [31].

In particular the Langevin approach has been applied to the study of the heavy quark energy loss by several groups, and the related physics is summarized in the review [31]. The Langevin evolution used was relativistic and with symmetric diffusion coefficients. As there was no microscopic model to provide the proper fluctuation-dissipation relation, the Einstein equations used vary, and in all examples it was assumed that the equilibrium distribution is the Jüttner-Boltzmann distribution. Moreover various combinations of friction forces were used, resonance models, $\mathrm{pQCD}, \mathrm{N}=4 \mathrm{AdS} / \mathrm{CFT}$ and combinations. A further recent analysis was performed in [32] with similar conclusions. The associated relativistic and isotropic Langevin systems used have been introduced in the mathematical physics literature rather recently, [33-35] (see [36] for a review).

The purpose of the present paper is to study further the relativistic Langevin evolution of a heavy quark using holographic techniques in a general context, and going beyond conformally invariant backgrounds characterized solely by AdS geometries. ${ }^{1}$ In this work we will study a large class of non-conformal backgrounds captured by Einstein-dilaton gravity with a dilaton potential in 5 dimensions. In a series of recent works, such backgrounds were analyzed both qualitatively and quantitatively and have provided a rich variety of holographic bulk dynamics. In particular, for a selected class of scalar potentials, they mimic the behavior of large- $N$ Yang Mills, [38]-[44]. This match can be quantitative, [44], agreeing very well both at zero and finite temperature with recent high-precision lattice data, [45]. On the other hand, the analysis we carry out in the present paper is general, as it applies to any asymptotically AdS background.

We therefore consider a fundamental string whose end-point lies in the UV region of a bulk black-hole background of a non-conformal holographic model. The string end-point is forced to move with velocity $v$. Solving the Nambu-Goto equations of motion, the classical profile of the trailing string can be found. The string stretches inside the bulk until it becomes completely horizontal at some value of radial coordinate $r_{s}$, given by $f\left(r_{s}\right)=v^{2}$ where $f(r)$ is the blackness function of the background. When the quark is moving slowly, as $v \rightarrow 0$, the point $r_{s}$ approaches the bulk black hole horizon.

The induced metric on the string world-sheet has the form of a two-dimensional blackhole metric with a horizon at $r=r_{s}$ as first observed in [19]. ${ }^{2}$ This black-hole is an

\footnotetext{
${ }^{1} \mathrm{~A}$ particular example in this class was recently considered in [24], which studied the Langevin process in the non-conformal $\mathcal{N}=2^{*}$ background.

${ }^{2}$ This is a generic effect on strings and D-branes embedded in black-hole/black-brane backgrounds. It was first observed in [46] where it was used to propose that a different speed of light is relevant for such branes. It is implicit or explicit in many holographic computations using probe flavor branes, [47, 48] and strings [19].
} 
important ingredient of the dynamics of the system. In particular it is crucial in the calculation of the thermal correlators using the Schwinger-Keldysh formalism, as well as for the fluctuation-dissipation relation. The world-sheet black hole has an associated Hawking temperature $T_{s}$ that depends on several parameters: the background temperature $T$, the zero-temperature bulk scale $\Lambda,{ }^{3}$ and the quark velocity $v$. It coincides with the temperature $T$ of the heat bath only in the non-relativistic limit. In the conformal case, one has $T_{s}=T_{\mathrm{s}, \mathrm{conf}}=T\left(1-v^{2}\right)^{\frac{1}{4}} \leq T$. The numerical analysis performed in section 6 shows that, in our non-conformal holographic model $T_{s} \leq T_{\mathrm{s}, \mathrm{conf}} \leq T$. The equality $T_{s}=T_{\mathrm{s} \text {,conf }}$, in the first relation is attained, for arbitrary $v$, in the high $\mathrm{T}$ limit, as shown in figure 1 in section 6 and also in the ultra-relativistic limit, $v \rightarrow 1$.

We next consider small fluctuations around the classical string profile. They satisfy second-order radial equations that are related to the associated thermal correlators by the holographic prescription. It should be emphasized that such correlators are thermal with temperature $T_{s}$ and not the temperature $T$ of the heat bath. ${ }^{4}$ Moreover, they satisfy the fluctuation-dissipation relation associated with the emergent temperature $T_{s}$. The fact that the string fluctuations see a modified temperature crucially affects the Einstein relation between the diffusion constants.

At the quadratic level of fluctuations, a relativistic Langevin diffusion equation is obtained using the AdS/CFT prescription. The diffusion constants and friction coefficients are calculated analytically in terms of the bulk metric, for general non-conformal backgrounds. The Einstein relation is now modified, due to the fact that the temperature is modified. Another important property is that the diffusion constants perpendicular and longitudinal to the motion (denoted by $\kappa_{\perp}$ and $\kappa_{\|}$) are different, a fact that was already observed in the conformal relativistic case, [22]. This is persisting here, and we are able to show, for general non-conformal backgrounds, that $\kappa_{\|} \geq \kappa_{\perp}$, namely the longitudinal diffusion constant always dominates the transverse one. Furthermore, both the diffusion constants and the friction term are momentum dependent, as expected. This is in contrast to the conformal case.

The properties of this relativistic Langevin evolution differ substantially from rotationally invariant equations that have been introduced recently in mathematical physics [32-36]. In particular, here the evolution is not rotationally symmetric, and the Einstein relation is different, because the fluctuation-dissipation relation is different. This implies that the equilibrium configuration is not the standard rotationally-invariant Jüttner-Boltzmann distribution.

The processes we discuss here are connected to a general property of a class of statistical systems, and provide a concrete and rather general solvable example thereof. Such systems, when in contact with a heat bath of temperature $T$, if stirred gently and continuously, end up in a stationary state that is thermal but with a temperature $T_{s}$, different from that of the heat bath. They satisfy a fluctuation-dissipation relation involving the new

\footnotetext{
${ }^{3}$ This appears as an integration constant in the background geometry and corresponds to the dynamically generated energy scale $\Lambda_{\mathrm{QCD}}$ in the dual field theory.

${ }^{4}$ More precisely put, the Langevin correlators that are obtained from the string fluctuations by the holographic prescription obey a modified Einstein relation with temperature $T_{s}$ rather than $T$.
} 
temperature, [49]. This phenomenon has been expected to occur in general, in situations with slow dynamics. These include in particular glassy systems, as well as systems that are very gently stirred by external agents and reach stationarity. Here, we have a system that is stationary but strongly driven by the external source (an electric field on the flavor brane, that keeps the velocity large and constant as the quark is moving through the plasma). Typically, $T_{s} \geq T$, [49] but in our case things are different. In the conformal case $T_{s}=T_{s, \text { conf }}=T\left(1-v^{2}\right)^{\frac{1}{4}} \leq T$ and this seems to persist in several other cases, as already mentioned before.

The problem we are solving can be also cast in a different light. The following question has been asked since the beginning of the 20th century: what are the Lorentztransformation properties of temperature and the associated stationary distribution? This question is still considered open, [50] with several conflicting answers. Our setup can be reconsidered as follows: the heavy quark is a "thermometer" moving inside a heat bath of temperature $T$. The way it measures temperature is via the fluctuation-dissipation theorem as argued in generality in [49]. Therefore the temperature that it measures as it moves in the thermal medium is $T_{s}$, which is velocity-dependent. This velocity dependence is simple in the conformal case, $T_{s}=T\left(1-v^{2}\right)^{\frac{1}{4}}$ but is rather complicated in the non-conformal case and is therefore system- (and possibly thermometer-) dependent.

The Langevin correlators must be renormalized as they are divergent near the AdS boundary of the string world-sheet. We show that the only UV divergence is subtracted by a counterterm that renormalizes the (heavy) quark mass. The associated scheme dependence affects the real part of the correlators only.

The local Langevin equation arises when looking at the large-time limit of the fluctuations of the heat bath, i.e. at the small frequency modes. On the other hand, the holographic computation gives access to the full frequency spectrum of the correlation functions driving the generalized Langevin dynamics. In this work we compute holographically the full Langevin correlators and the associated spectral densities. In particular, we obtain analytic expressions (in terms of the bulk metric and dilaton profiles) in the two opposite regimes of small and large frequencies $\omega$ (compared to an appropriate temperature scale).

For small frequencies, an analytic expression for the spectral densities is obtained using the membrane paradigm [51], which allows to relate these quantities to the near-horizon values of the background functions. In the large-frequency regime, on the other hand, the spectral densities are obtained via a modified WKB method, similar to the one followed in [52] for bulk fluctuations in an AdS-Schwarzschild background. The high-frequency behavior is different, depending on the mass of the probe quark. For finite mass, and for large $\omega$, the spectral densities grows linearly with $\omega$, whereas in the limit when the quark mass becomes infinite this behavior changes to a cubic power-law.

Going beyond the zero-frequency limit is necessary when the diffusion process happens on time scales comparable to, or smaller than the auto-correlation time of the fluctuation propagators. More specifically, since these are thermal correlators at the temperature $T_{s}$, the large-time approximation breaks down over time-scales shorter than $T_{s}^{-1}$. This condition puts a temperature-dependent upper bound on the momenta of the heavy quark, above which the diffusion process cannot be described by a simple local Langevin equation 
with white noise. In this context, it is useful to have approximate expressions for valid for large frequencies (for examples, those we obtain with the WKB method) to model the behavior of the system in the regime where the local Langevin approximation breaks down and the dynamics becomes non-markovian (due to a non-trivial memory kernel)

The results described above apply to any five-dimensional holographic model which admits asymptotically AdS black-hole solutions. On the other hand, it is interesting to perform a quantitative comparison between the diffusion constants calculated in concrete models, and characteristic observables in heavy ion experiments that can simply be connected to Langevin processes. In the context of heavy-ion physics, the transverse diffusion constant is directly related to the jet-quenching parameter $\hat{q}_{\perp}=2 \kappa_{\perp} / v$. The latter is a convenient quantity to describe the observed phenomenon of transverse momentum broadening of a heavy quark: this is the process by which the transverse momentum of the heavy quark probe ${ }^{5}$ initially equal to zero, undergoes a stochastic diffusion process such that after a time $t$ it acquires a dispersion $\Delta p_{\perp}^{2}=\hat{q}_{\perp} v t$.

In the final part of this work we perform a quantitative analysis of both the full Langevin correlators, and of the jet-quenching parameter, in a particular Einstein-dilaton model, namely Improved Holographic QCD [38, 39], which agree quite well both qualitatively and quantitatively with the zero- and finite-temperature Yang-Mills theory. In particular, we focus on the specific model which was put forward in [44], and displays a good quantitative match with the spectral and thermodynamic properties of lattice Yang Mills theory.

The analysis is performed numerically, both with respect to the background metric and to the solution of the fluctuation equations. By a shooting technique, we determine the wave-functions describing the world-sheet fluctuations, and obeying the appropriate retarded boundary conditions. From the wave-functions, the holographic prescription allows to determine the full Langevin retarded correlator, whose imaginary part gives the associated spectral density.

Using the exact numerical evaluation we are able to test the different analytic results discussed above. In particular, we test the validity of the WKB result for large frequency, and in various regimes of quark mass and velocity. Unexpectedly, we find that the analytic WKB formulae not only capture the large frequency regime, but are a very good approximation to the correlators at almost all frequencies.

The numerical evaluation of the diffusion constants may lead directly to a comparison of the jet-quenching parameters between the holographic QCD model and data. We find that $\hat{q}_{\perp}$ displays a mild momentum dependence for large quark momenta, which however differs from the one obtained holographically in the conformal case. As the temperature rises, $\hat{q}_{\perp}$ increases significantly, approximately as $\sim T^{3}$. Interestingly, it is found that for temperatures above $\sim 400 \mathrm{MeV}$, the local description of the diffusive process breaks down for charm quarks with momenta above $\sim 5-10 \mathrm{GeV}$. This is because the process occurs on time scales shorter than $1 / T_{s}$. This would imply that in order to describe heavy charm quark diffusion in the ALICE experiment, one would need the full generalized non-local

\footnotetext{
${ }^{5}$ Here, "transverse" refers to the initial quark trajectory, not to the direction of the colliding beams.
} 
Langevin equation, and the full frequency-dependent correlator, rather than just its lowfrequency limit captured by $\hat{q}_{\perp}$. This would constitute an interesting testing ground for holographic models, where the full correlators can be easily computed. We also estimate the energies at which the energy loss mechanism described here, is not any more the dominant one and radiation becomes the dominant mechanism. This is estimated by requiring that $r_{s}$ remains below the would-be position of the flavor brane, $[19,59]$. We show that these limit do not substantially constrain this framework.

A direct quantitative comparison of the results of this paper with data is hampered by the fact that quark degrees of freedom in the plasma are not included in our analysis. We are compensating (partly at least) for this using the "energy scheme" for comparison, however the recent results found in [60] (and reviewed in [61]) suggest that even in that case we may be underestimating the result.

In summary the AdS/CFT calculation of the Langevin diffusion of heavy quarks has the following characteristics

- The diffusion coefficients are asymmetric. The longitudinal diffusion coefficients is always larger than the transverse one. They both become large with increasing $\gamma$.

- The Langevin correlators satisfy a thermal fluctuation-dissipation relation with a temperature $T_{s}$ that is typically smaller than then heat bath temperature $T$. In the conformal case $T_{s}=\frac{T}{\sqrt{\gamma}},[22]$. The associated Einstein relations are non-standard, especially the longitudinal one.

- The local (Markovian) Langevin diffusion breaks down at some energy scale. Beyond this scale, the full force correlators are needed. This breakdown is expected to be relevant at LHC energies for the charm.

This paper is organized as follows. Section 2 describes in detail the Langevin equation for a relativistic heavy quark travelling in the quark-gluon plasma. In this section we also review how to describe the Langevin dynamics in the holographically dual geometry, in terms of fluctuations of trailing strings. Section 3 presents the necessary background for the holographic computation. In particular we present the holographic dual geometry of our non-conformal model, the relevant classical trailing string solution, and the corresponding linear fluctuations. It is in this section that we obtain the fluctuation equations in general non-conformal black hole space-times, whose solutions enter the construction of the Langevin propagators.

Sections 4 and 5 contain our main results. In section 4 we discuss the Langevin correlators and the associated spectral densities, first in full generality, then in the various limits of low- and high- frequency. In section 5 we specialize to the low-frequency modes, which compute the long-time behavior of the diffusion and friction coefficients of the local Langevin equation. We provide exact analytic expressions for these quantities, in terms of the background metric functions. We also discuss the non-relativistic and ultra-relativistic limits, and derive the modified Einstein relations. While the previous sections deal with a completely general holographic dual, in section 6 we provide a numerical study of these 
results in a specific model, namely Improved Holographic QCD that was shown in [44] to provide a good quantitative description of the static properties of pure Yang-Mills at zero and finite temperature. In particular, in this section we compute the jet-quenching parameter arising from this model, and discuss the results in light of RHIC data.

Several technical details are left to the appendices. In appendix A we discuss some subtleties in the definition of the propagator, related to boundary terms. Appendix B provides the details of the calculation of the diffusion constants; In appendix $\mathrm{C}$ we give a detailed discussion of the WKB method that we use to obtain the large-frequency limit of the spectral densities, as it is more involved than the conformal case. In appendix D we discuss the Langevin correlators in the conformal, $\mathcal{N}=4$ case.

\section{Langevin equation for a relativistic heavy quark}

In this section we review how the diffusion of a relativistic heavy quark through the plasma is described by a generalized Langevin equation. First we give the purely $4 \mathrm{D}$ picture. Then, in subsection 4.2, we review the holographic description of the Langevin process that appeared in the previous literature, for the case of AdS-Schwarzschild black-holes. This will be extended to general asymptotically AdS geometries in section 3 .

\subsection{The Langevin equation in the boundary theory}

Consider a quark which, in a first approximation, experiences a uniform motion across the plasma, with constant velocity $v$. Due to the interactions with the strongly-coupled plasma, the actual trajectory of the quark is expected to resemble Brownian motion. To lowest order, the action for the external quark coupled to the plasma can be assumed, classically, to be of the form:

$$
S[X(t)]=S_{0}+\int d \tau X_{\mu}(\tau) \mathcal{F}^{\mu}(\tau)
$$

where $S_{0}$ is the free quark action, and $\mathcal{F}(\tau)$ depends only on the plasma degrees of freedom, and plays the role of a driving force (the "drag" force).

To obtain an equation for the quark trajectory one needs to trace over the plasma degrees of freedom. If the interaction energies are small compared with the quark kinetic energy (therefore for a very heavy quark, and/or for ultra-relativistic propagation speeds), tracing over the microscopic degrees of freedom of the plasma can be performed in the semiclassical approximation, and the quark motion can be described by a classical generalized Langevin equation for the position $X^{i}(t)$, of the form:

$$
\frac{\delta S_{0}}{\delta X_{i}(t)}=\int_{-\infty}^{+\infty} d \tau \theta(\tau) C^{i j}(\tau) X_{j}(t-\tau)+\xi^{i}(t), \quad i=1,2,3
$$

Here, $C^{i j}(t)$ is a memory kernel, $\theta(\tau)$ is the Heaviside function and $\xi(t)$ is a Gaussian random variable with time-correlation:

$$
\left\langle\xi^{i}(t) \xi^{j}\left(t^{\prime}\right)\right\rangle=A^{i j}\left(t-t^{\prime}\right)
$$


The functions $A^{i j}(t)$ and $C^{i j}(t)$ are determined by the symmetrized and antisymmetrized real-time correlation functions of the forces $\mathcal{F}(t)$ over the statistical ensemble:

$$
C^{i j}(t)=G_{\text {asym }}^{i j}(t) \equiv-i\left\langle\left[\mathcal{F}^{i}(t), \mathcal{F}^{j}(0)\right]\right\rangle, \quad A^{i j}(t)=G_{\mathrm{sym}}^{i j}(t) \equiv-\frac{i}{2}\left\langle\left\{\mathcal{F}^{i}(t), \mathcal{F}^{j}(0)\right\}\right\rangle .
$$

The results (2.2) and (2.4) are very general, and do not require any particular assumption about the statistical ensemble that describes the medium (in particular, they do not require thermal equilibrium). One way to arrive at equation (2.2) is using the double time formalism and the Feynman-Vernon influence functional [54]. A clear and detailed presentation can be found in [55], chapter 18.

The retarded and advanced Green's function are defined by:

$$
G_{R}^{i j}(t)=\theta(t) C^{i j}(t), \quad G_{A}^{i j}(t)=-\theta(-t) C^{i j}(t),
$$

which lead to the relation

$$
C^{i j}(t)=G_{R}^{i j}(t)-G_{A}^{i j}(t)
$$

Notice that the kernel entering the first term on the right in equation (2.2) is the retarded Green's function, $G_{R}^{i j}(t)=\theta(t) C^{i j}(t)$.

It is customary to introduce a spectral density $\rho^{i j}(\omega)$ as the Fourier transform of the anti-symmetrized (retarded) correlator,

$$
C^{i j}(t)=-i \int_{-\infty}^{+\infty} d \omega \rho^{i j}(\omega) e^{-i \omega t}, \quad G_{R}^{i j}(\omega)=\int_{-\infty}^{+\infty} d \omega^{\prime} \frac{\rho^{i j}\left(\omega^{\prime}\right)}{\omega-\omega^{\prime}+i \epsilon} .
$$

From equation (2.6) and the reality condition $G_{A}(t)=G_{R}(-t)$, or in Fourier space, $G_{A}(\omega)=G_{R}^{*}(\omega)$, we can relate the spectral density to the imaginary part of the retarded correlator:

$$
\rho^{i j}(\omega)=-\frac{1}{\pi} \operatorname{Im} G_{R}^{i j}(\omega)
$$

Local limit. Suppose the time-correlation functions vanish for sufficiently large separation, i.e. for times much larger than a certain correlation time $\tau_{c}$. Then, in the limit $t \gg \tau_{c}$, equation (2.2) becomes a conventional local Langevin equation, with local friction and white noise stochastic term. Indeed, in this regime the noise correlator can be approximated by

$$
A^{i j}\left(t-t^{\prime}\right) \approx \kappa^{i j} \delta\left(t-t^{\prime}\right), \quad t-t^{\prime} \gg \tau_{c}
$$

This equation defines the Langevin diffusion constants $\kappa^{i j}$. Similarly, for the friction term, we define the function $\gamma^{i j}(t)$ by the relation:

$$
C^{i j}(t)=\frac{d}{d t} \gamma^{i j}(t)
$$

so that the friction term can be approximated, for large times, as:

$$
\int_{0}^{\infty} d \tau C^{i j}(\tau) X_{j}(t-\tau) \approx\left(\int_{0}^{\infty} d \tau \gamma^{i j}(\tau)\right) \dot{X}_{j}(t), \quad t \gg \tau_{c} .
$$


In this regime, equation (2.2) becomes the local Langevin equation with white noise,

$$
\frac{\delta S_{0}}{\delta X_{i}(t)}+\eta^{i j} \dot{X}_{j}(t)=\xi^{i}(t), \quad\left\langle\xi^{i}(t) \xi^{j}\left(t^{\prime}\right)\right\rangle=\kappa^{i j} \delta\left(t-t^{\prime}\right),
$$

with the self-diffusion and friction coefficients given by:

$$
\kappa^{i j}=\lim _{\omega \rightarrow 0} G_{\mathrm{sym}}^{i j}(\omega) ; \quad \eta^{i j} \equiv \int_{0}^{\infty} d \tau \gamma^{i j}(\tau)=-\lim _{\omega \rightarrow 0} \frac{\operatorname{Im} G_{R}^{i j}(\omega)}{\omega} .
$$

In the case of a system at equilibrium with a canonical ensemble at temperature $T$, one has the following relation between the Green's functions:

$$
G_{\mathrm{sym}}(\omega)=-\operatorname{coth} \frac{\omega}{2 T} \operatorname{Im} G_{R}(\omega),
$$

which using equation (2.13) leads to the Einstein relation $\kappa^{i j}=2 T \eta^{i j}$. For such a thermal ensemble, the real-time correlators decay exponentially with a scale set by the inverse temperature, therefore the typical correlation time is $\tau_{c} \sim 1 / T$.

Determining and studying the Langevin correlators (2.4), and the diffusion constants (2.12) will be the main purpose of the rest of this paper.

Next, we write down explicitly the classical part, $\delta S_{0} / \delta X(t)$ of the Langevin equation, in order to arrive at an equation describing momentum diffusion. We start with the kinetic action for a free relativistic quark,

$$
S_{0}\left[X_{\mu}(\tau)\right]=-M_{q} \int d \tau \sqrt{\frac{d X^{\mu}}{d \tau} \frac{d X_{\mu}}{d \tau}}
$$

We choose the gauge $\tau=X^{0}$, and obtain

$$
\delta S_{0} / \delta X^{i}(\tau)=d p_{i} / d t \quad, \quad \text { with } \quad, \quad p_{i} \equiv M_{q} \dot{X}_{i}\left(1-\dot{X}_{i} \dot{X}^{i}\right)^{-1 / 2} .
$$

Equation (2.12) becomes the Langevin equation for momentum diffusion:

$$
\frac{d p^{i}}{d t}=-\eta_{D}^{i j}\left(\vec{p}^{2}\right) p_{j}+\xi^{i}(t)
$$

where:

$$
\eta_{D}^{i j}\left(\vec{p}^{2}\right)=\frac{\eta^{i j}}{\gamma\left(\vec{p}^{2}\right) M_{q}}, \quad \gamma\left(\vec{p}^{2}\right) \equiv \sqrt{1+\vec{p}^{2} / M_{q}^{2}} .
$$

Linearized Langevin equations. For a generic quark trajectory, the Langevin equation (2.17) is non-linear, due to the $p$-dependence implicit in $\eta_{D}^{i j}$. To put it in a form which allows for the holographic treatment in terms of the trailing string fluctuations, it is convenient to derive from equation (2.17) a linearized Langevin equation for the fluctuations in the position around a trajectory with uniform velocity, $\vec{X}(t)=\vec{v} t+\delta \vec{X}$. To this end, we separate the longitudinal and transverse components of the velocity fluctuations:

$$
\dot{\vec{X}}(t)=\left(v+\delta \dot{X}^{\|}(t)\right) \frac{\vec{v}}{v}+\delta \dot{\vec{X}}^{\perp}
$$


The corresponding linearized expression of the momentum reads:

$$
\vec{p}=M_{q} \frac{\dot{\vec{X}}}{\sqrt{1-\dot{\vec{X}} \cdot \dot{\vec{X}}}} \simeq M_{q}\left(\gamma+\gamma^{3} v \delta \dot{X}^{\|}\right)(\vec{v}+\delta \dot{\vec{X}})=\overrightarrow{p_{0}}+\delta \vec{p},
$$

where we introduced the zeroth-order Lorentz factor $\gamma \equiv\left(1-v^{2}\right)^{-1 / 2}$. The zeroth-order term is $\vec{p}_{0}=\gamma M_{q} \vec{v}$, and the longitudinal and transverse momentum fluctuations are given by:

$$
\delta p^{\|}=\gamma M_{q}\left(1+v^{2} \gamma^{2}\right) \delta \dot{X}^{\|}=\gamma^{3} M_{q} \delta \dot{X}^{\|}, \quad \delta p_{i}^{\perp}=\gamma M_{q} \delta \dot{X}^{\perp} .
$$

It is convenient to separate the longitudinal and transverse components of the propagators, since as it will become clear in the next section, the off-diagonal components vanish:

$$
G^{i j}(t)=G^{\|}(t) \frac{v^{i} v^{j}}{v^{2}}+G^{\perp}(t)\left(\delta^{i j}-\frac{v^{i} v^{j}}{v^{2}}\right)
$$

and the corresponding decompositions for $\eta^{i j}$ and $\kappa^{i j}$ from (2.13).

Inserting these expressions in equation (2.17), we find to zeroth order:

$$
\frac{d p_{0}}{d t}=-\eta_{D}^{\|} p_{0}, \quad p_{0} \equiv \gamma M v
$$

and to first order in $\delta \vec{X}$ the relativistic Langevin equations for position fluctuations:

$$
\begin{aligned}
\gamma^{3} M_{q} \delta \ddot{X}^{\|} & =-\eta^{\|}(v) \delta \dot{X}^{\|}+\xi^{\|}, & \left\langle\xi^{\|}(t) \xi^{\|}\left(t^{\prime}\right)\right\rangle & =\kappa^{\|} \delta\left(t-t^{\prime}\right), \\
\gamma M_{q} \delta \ddot{X}^{\perp} & =-\eta^{\perp}(v) \delta \dot{X}^{\perp}+\xi^{\perp}, & \left\langle\xi^{\perp}(t) \xi^{\perp}\left(t^{\prime}\right)\right\rangle & =\kappa^{\perp} \delta\left(t-t^{\prime}\right)
\end{aligned}
$$

where the friction coefficients $\eta^{\|, \perp}$ are related to the coefficients $\eta_{D}^{i j}$ by

$$
\eta^{\perp}=\gamma M_{q} \eta_{D}^{\perp}, \quad \eta^{\|}=\gamma^{3} M_{q}\left(\eta_{D}^{\|}+\left.p \frac{\partial \eta_{D}^{\|}}{\partial p}\right|_{p=\gamma M_{q} v}\right) .
$$

As we shall see in the following sections, the holographic prescription will directly compute the friction coefficients $\eta^{i j}$ and the diffusion coefficients $\kappa^{i j}$ appearing in equations $(2.24)-(2.25)$.

Short-time solution: momentum broadening. For times shorter than the relaxation time $\tau_{D} 1 / \eta_{D}$ we can treat the quark as travelling at a constant velocity $v$ ( which is a good approximation in the case of a very heavy quark). In this regime, ${ }^{6}$ one can write an approximate solution for equation (2.17), which describes a Brownian-like diffusion for momentum fluctuations.

We start once again with equation (2.17), and linearize it (this time staying in momentum space) around a uniform trajectory $\vec{p} \simeq p_{0} \vec{v} / v+\delta \vec{p}$. In the longitudinal and transverse directions we find the two equations:

$$
\frac{d \delta p^{\perp}}{d t}=-\eta_{D, 0}^{\perp} \delta p^{\perp}+\xi^{\perp}, \quad \frac{d \delta p^{\|}}{d t}=-\eta_{D, 0}^{\|} p_{0}+\left[\eta_{D}^{\|}+p\left(\frac{\partial \eta_{D}^{\|}}{\partial p}\right)\right]_{p_{0}} \delta p^{\|}+\xi^{\|}
$$

\footnotetext{
${ }^{6}$ As we are relying on the local form of the Langevin process, equation (2.17), we must still require time separations much larger than the auto-correlation time $\tau_{c}$. More explicitly, we consider time scales $t$ such that $\tau_{c} \ll t \ll \tau_{D}$. Therefore, consistency demands that $\tau_{D} \gg \tau_{c}$.
} 
where $\eta_{D, 0}^{i j} \equiv \eta_{D}^{i j}\left(p_{0}\right)$. The solution to these equations is straightforward: assuming initial conditions $\delta \vec{p}(t=0)=0$, it reads (notice that $p^{\perp}=\delta p^{\perp}$ ):

$$
\begin{aligned}
& p^{\perp}(t)=\int_{0}^{t} d t^{\prime} e^{\eta_{D, 0}^{\perp}\left(t^{\prime}-t\right)} \xi^{\perp}\left(t^{\prime}\right), \\
& p^{\|}(t)=p_{0} e^{-\eta_{D, 0}^{\|} t}+\int_{0}^{t} d t^{\prime} e^{\tilde{\eta}_{D, 0}^{\|}\left(t^{\prime}-t\right)} \xi^{\|}\left(t^{\prime}\right), \quad \tilde{\eta}_{D, 0}^{\|} \equiv\left[\eta_{D}^{\|}+p\left(\frac{\partial \eta_{D}^{\|}}{\partial p}\right)\right]_{p_{0}} .
\end{aligned}
$$

From these solutions, we can compute the noise-average of the transverse and longitudinal momentum fluctuations

$$
\begin{aligned}
& \left\langle\left(p^{\perp}\right)^{2}\right\rangle=\int_{0}^{t} d t^{\prime} \int_{0}^{t} d t^{\prime \prime} e^{\eta_{D}^{\perp}, 0}\left(t^{\prime}+t^{\prime \prime}-2 t\right)\left\langle\xi^{\perp}\left(t^{\prime}\right) \xi^{\perp}\left(t^{\prime \prime}\right)\right\rangle \\
& \left\langle\left(p^{\|}-p_{0}\right)^{2}\right\rangle=p_{0}^{2}\left(1-e^{-\eta_{D, 0}^{\|} t}\right)^{2}+\int_{0}^{t} d t^{\prime} \int_{0}^{t} d t^{\prime \prime} e^{\tilde{\eta}^{\|}\left(t^{\prime}+t^{\prime \prime}-2 t\right)}\left\langle\xi^{\|}\left(t^{\prime}\right) \xi^{\|}\left(t^{\prime \prime}\right)\right\rangle .
\end{aligned}
$$

Using the fact that $\left\langle\xi\left(t^{\prime}\right) \xi\left(t^{\prime \prime}\right)\right\rangle=\kappa \delta\left(t^{\prime}-t^{\prime \prime}\right)$, and expanding to linear order in $t \eta_{D} \ll 1$, we arrive at the final result:

$$
\left\langle\left(p^{\perp}\right)^{2}\right\rangle=2 \kappa^{\perp} t, \quad\left\langle\left(\Delta p^{\|}\right)^{2}\right\rangle=\kappa^{\|} t .
$$

The first equation describes transverse momentum broadening, and it is typically parametrized in terms of the jet-quenching parameter $\hat{q}^{\perp}$,

$$
\hat{q}^{\perp}=\frac{\left\langle\left(p^{\perp}\right)^{2}\right\rangle}{v t}=2 \frac{\kappa^{\perp}}{v}
$$

The following sections, namely 4,5 , and 6 will be devoted to the calculation, in a 5D holographic setup, of the Langevin correlators (2.4) and of the diffusion constants $\kappa^{\|}$and $\kappa^{\perp}$ appearing in equation (2.17).

\subsection{The Langevin equation in the gravity dual picture}

As we have reviewed in the previous subsection, the memory kernel and the noise timecorrelation function that govern the generalized Langevin equation (2.2) for an external quark, are given by appropriate real-time correlation functions of the force operator $\mathcal{F}(t)$ over the ensemble that describes the medium.

These correlation functions are precisely the kinds of objects one can compute in the gravity dual picture: one needs to identify the appropriate bulk field that couples to the boundary operator $\mathcal{F}$, then solve the bulk equations for this field with appropriate boundary conditions.

As first discussed in [10-13], and as we will review in detail in section 3, a probe heavy quark propagating through the plasma is described, in the gravity dual picture, by a probe string with an endpoint attached to a flavor brane, and extending into the bulk. The string endpoint moves along the quark trajectory and the rest of the string trails its endpoint extending in the holographic directions. The string world-sheet is described by 
the embedding coordinates $X^{A}(\sigma, \tau)$ which, in the static gauge $\tau=t, \sigma=r$, reduce to the spacial components $\vec{X}(r, t)$, where $r$ is the non-compact holographic direction.

Using the trailing string picture for the heavy quark, the identification of the appropriate bulk field is straightforward: from equation (2.1) it is clear that the external source for the boundary field $\mathcal{F}_{i}(t)$ is nothing but the quark position $X^{i}(t)$, i.e. the boundary value for the string embedding $X^{i}(t, r)$. More precisely, for a heavy quark that follows an approximately uniform trajectory $X^{i}(t)=v^{i} t+\delta X^{i}(t)$ the boundary coupling is of the form

$$
S_{\text {coupling }}=\int d t \delta X^{i}(t) \mathcal{F}_{i}(t), \quad \delta X^{i}(t)=\delta X\left(r_{b}, t\right)
$$

where $\delta X\left(r_{b}, t\right)$ is the boundary value of the fluctuation in the trailing string around the classical profile. Therefore, the correlation functions (2.4) of the force operators can be extracted, in the Gaussian approximation, by solving for the bulk linear fluctuations around the trailing string and using the appropriate holographic prescription.

This calculation was first performed in $[16,18]$, for the AdS case. In this case, the world-sheet fluctuations propagate on a space-time with a metric of the form (3.30), with

$$
b(r)=\ell / r, \quad f(r)=1-(\pi T r)^{4},
$$

where $T$ is the black-hole temperature. For a quark velocity $v$, the induced metric has a horizon at $0<r_{s}<r_{h}$, with associated temperature $T_{s}=T / \sqrt{\gamma}$. The retarded correlator for the longitudinal and transverse components of $\overrightarrow{\mathcal{F}}$ was determined using the prescription of [57]:

$$
\gamma^{-2} G_{R, \mathrm{AdS}}^{\|}=G_{R, \mathrm{AdS}}^{\perp}=-\left.\Psi^{*} \mathcal{G}^{r r} \partial_{r} \Psi\right|_{\text {Boundary }} .
$$

where $\Psi(r, \omega)$ is a solution to the fluctuation equation with unit normalization at the boundary and infalling boundary conditions at the horizon, $\mathcal{G}^{r r}=\left(2 \pi \ell_{s}^{2}\right)^{-1} H^{r r}$, with $H^{r r}$ given by equation (3.35) specialized to the AdS-Schwarzschild case.

In order to compute both terms entering the Langevin equation, one needs also the symmetrized correlator, which gives the noise time-correlation function. In general, the relation between the retarded and symmetrized correlator depends on the statistical ensemble one is dealing with. For a black hole (as in the case of the induced world-sheet metric), the features of the ensemble can be obtained by connecting the mode solutions along a Keldysh contour between the two boundaries of the maximally extended Kruskal diagram. This corresponds to obtaining a statistical ensemble by tracing over the degrees of freedom of one of the causally disconnected regions. In the context of AdS/CFT this idea was put forward in [17], which also provides a justification of the prescription (2.36) for the retarded propagator.

We will not go into the details of this procedure, which can be found in $[18,19]$. The crucial point is that the stationary statistical ensemble one obtains is a thermal ensemble at the temperature $T_{s}=T / \sqrt{\gamma}$. Therefore, one can compute $G_{\text {sym }}$ from the imaginary part of $G_{R}$, as in equation (2.14), with the substitution $T \rightarrow T / \sqrt{\gamma}$.

Notice that the retarded Green's functions compute, through equations (2.13), the coefficients appearing in the Langevin equations for the fluctuations $\delta X^{i}$, equa- 
tion (2.24)-(2.25). In particular, to extract the coefficient $\eta_{D}^{\|}$one must divide $G^{\|}(\omega)$ by an extra factor $\gamma^{2}$ with respect to the corresponding result for $\eta_{D}^{\perp}$.

Computing the zero-frequency limit of the retarded correlators, the resulting Langevin diffusion coefficients are found to be $[18,19]$ :

$$
\gamma^{-2} \kappa^{\|}=\kappa^{\perp}=\pi \frac{\ell^{2}}{\ell_{s}^{2}} \sqrt{\gamma} T^{3},
$$

and the friction coefficients reproduce the classical drag force calculation [13],

$$
\eta_{D}^{\|}=\eta_{D}^{\perp}=\frac{\pi}{2} \frac{\ell^{2}}{\ell_{s}^{2}} \frac{T^{2}}{M}
$$

The diffusion and friction coefficients indeed satisfy an Einstein relation appropriate for the temperature $T_{s}$ :

$$
\kappa^{\perp} / \eta^{\perp}=\kappa^{\|} / \eta^{\|}=2 M T_{s},
$$

where $\eta^{\perp}$ and $\eta^{\|}$are related to $\eta_{D}$ by equation (2.26). Notice that, in the conformal case, $\eta_{D}$ is momentum-independent.

The approach taken in [18] derives the Langevin propagators by using the standard holographic prescription for real-time correlators. An alternative procedure, giving the same result, was adopted in [20, 21] for the non-relativistic case, and later in [22] for the general relativistic case. These authors performed a direct derivation of the Langevin equation: starting from the trailing string fluctuations in the bulk, and integrating them out, they showed explicitly that one arrives at equations like (2.24)-(2.25) for the boundary fluctuations, with coefficients given by the formulae previously found in $[16,18]$. Furthermore, they showed that the same result can be obtained by integrating out only a strip between the world-sheet horizon $r_{s}$ and the stretched horizon $r_{s}+\epsilon$ : this gives a picture of the stochastic behavior of the string fluctuations as originating from the world-sheet horizon. In this work we will not follow explicitly this road, but rather rely on the holographic computation of the Langevin correlators. Nevertheless, by using the general formalism developed [51] we will compute directly the transport coefficients.

\section{5D non-conformal backgrounds for Langevin holography}

In this section, we present the background material for the holographic computation of the Langevin correlators that we carry out in the section 4. As a bulk geometry we take a general, five-dimensional, asymptotically AdS black-hole, dual to a non-conformal deconfined plasma. These geometries arise generically as solutions in appropriate Einsteindilaton theories in five-dimensions [41, 42].

A heavy external quark moving through the plasma at temperature $T$ can be described by a string whose endpoint at the boundary follows the quark's trajectory [10]-[37]. The string extends into the bulk, whose geometry is the dual black hole background with appropriate temperature $T$. Once the motion of the endpoint at the boundary is specified, one can find the trailing string solution through the geodesic equation: the momentum flow along the string is dual to the drag force experienced by the quark moving through the plasma. 
The fluctuations of the string world-sheet around the geodesic solution are holographically dual to the stochastic forces felt by the quark due to its interaction with the medium. Their effect to leading (Gaussian) order is that of stochastic noise acting on the quark, resulting in a Langevin-type diffusion with its associated transport coefficients (diffusion constants).

\subsection{D Einstein-Dilaton black holes}

We shall consider the dynamics of a probe string in a general 5D black hole geometry, with a string frame metric:

$$
d s^{2}=b^{2}(r)\left[\frac{d r^{2}}{f(r)}-f(r) d t^{2}+d x^{i} d x_{i}\right]
$$

We assume there is an asymptotically AdS region $r \rightarrow 0$ where

$$
\log b(r) \sim-\log \frac{r}{\ell}+\text { subleading, } \quad f(r) \sim 1+O\left(r^{4}\right), \quad r \rightarrow 0,
$$

and a horizon at $r=r_{h}$ where $f\left(r_{h}\right)=0$, and $f^{\prime}\left(r_{h}\right)$ and $b\left(r_{h}\right)$ remain finite. The black hole temperature is given by:

$$
4 \pi T=-f^{\prime}\left(r_{h}\right)
$$

We make no particular assumptions on the subleading terms in equation (3.2). In case these subleading terms actually vanish as $r \rightarrow 0$, then the metric is AdS in the usual sense.

The black holes of the type (3.1) arise, in particular, as solutions of a large class of 5 -dimensional Einstein-dilaton models, described by the Einstein-frame metric $g_{\mu \nu}^{E}$ and a scalar field $\lambda$ with the action:

$$
S=-M_{p}^{3} N_{c}^{2} \int \sqrt{-g^{E}}\left[R^{E}-\frac{4}{3} \frac{(\nabla \lambda)^{2}}{\lambda^{2}}+V(\lambda)\right] .
$$

In the holographic interpretation of these models, the scalar $\lambda$ is dual to the running coupling $\lambda_{t}$ of the four-dimensional gauge theory. This is the class of models we will have in mind, although the results of this work apply to any 5D theory that admits solutions such as (3.1).

For an appropriate choice of the potential $V(\lambda)$, the models with action (3.4) provide a good holographic dual to large- $N_{c} 4$-dimensional pure Yang-Mills theory, at zero and finite temperature [38]-[44]. The potential should have a regular expansion as $\lambda \rightarrow 0$, with

$$
V(\lambda) \sim \frac{12}{\ell^{2}}\left(1+v_{0} \lambda+\ldots\right) .
$$

Furthermore, linear confinement in the IR requires that, at large $\lambda, V(\lambda)$ grows at least as fast as $\lambda^{4 / 3}$. With these requirements,

1. The solutions in the Einstein frame are an asymptotically AdS metric, with AdS length $\ell$, and a non-trivial profile $\lambda(r)$;

2. There is a first order Hawking-page phase transition with a non-zero critical temperature $T_{c}$. 
For a short review of the main features of these models, the reader is referred to [9].

We will be interested in the fluctuations of a probe string in the $5 \mathrm{D}$ black hole geometry for $T>T_{c}$ (corresponding to the deconfined phase). The black hole solutions in the string frame have the form (3.1), with string frame metric $g_{\mu \nu}=\lambda^{4 / 3} g_{\mu \nu}^{E}$. In the UV region $r=0$, we therefore have:

$$
b(r) \sim \frac{\ell}{r} \lambda^{2 / 3}(r), \quad f(r) \sim 1-\frac{\mathcal{C}}{\ell^{3}} r^{4}, \quad \lambda(r) \sim-\frac{9}{8 v_{0} \log r} .
$$

where the constant $\mathcal{C}$ depends on the thermodynamic quantities that characterize the black hole, and it can be expressed in terms of the temperature $T$ and entropy density $s$, [42]:

$$
\frac{\mathcal{C}}{\ell^{3}}=\frac{45 \pi^{2}}{N_{c}^{2}} s T
$$

The expressions (3.6) are corrected by terms of $\mathcal{O}(\lambda)$, which are negligible near the boundary.

\subsection{Classical trailing string and the drag force}

Before going into the details of the world-sheet fluctuations, we review the calculation of the unperturbed trailing solution, that was discussed in $[10,13]$ for pure AdS black holes, and generalized in [15] for black holes in 5D Einstein-Dilaton theories. In this subsection we review the setup and the results of [15].

We consider an (external) heavy quark moving through an infinite volume of gluon plasma with a fixed velocity $v$ at a finite temperature $T$. In the dual picture, this is described by a classical "trailing" string with an endpoint on the UV boundary moving at constant velocity $v$.

The world-sheet of the string is described by the Nambu-Goto action, ${ }^{7}$

$$
S_{\mathrm{NG}}=-\frac{1}{2 \pi \ell_{s}^{2}} \int d^{2} \sigma \sqrt{-\operatorname{det} g_{\alpha \beta}}, \quad g_{\alpha \beta}=g_{\mu \nu} \partial_{\alpha} X^{\mu} \partial_{\beta} X^{\nu}, \quad\left\{\begin{array}{c}
\mu, \nu=0 \ldots 5 \\
\alpha, \beta=0,1
\end{array}\right.
$$

where $g_{\mu \nu}$ are the components of the bulk metric in the string frame, ${ }^{8}$

$$
d s^{2}=b^{2}(r)\left[\frac{d r^{2}}{f(r)}-f(r) d t^{2}+d x^{i} d x^{i}\right], \quad i=1,2,3 .
$$

The ansatz for the classical trailing string is [13],

$$
X^{1}=v t+\xi(z), \quad X^{2}=X^{3}=0,
$$

\footnotetext{
${ }^{7}$ Throughout the paper, we will denote $5 \mathrm{D}$ coordinates by $\mu, \nu \ldots$, world-sheet coordinates by $\alpha, \beta \ldots$, and boundary spatial coordinates by $i, j \ldots$ Indices $i, j \ldots$ in the boundary theory are raised and lowered with metric $\delta_{i j}$, so we will make no distinctions between upper and lower indices as far as boundary tensors are concerned.

${ }^{8}$ Unless otherwise stated, $b(r)$ will always denote the scale factor in the string frame. This is a slight change of notation with respect to our previous papers [38, 39, 41, 42, 44], where the same quantity was denoted $b_{s}(r)$, but it is justified since most expressions in this work are simpler in the string frame.
} 
and along with the gauge choice

$$
\xi^{0}=t, \quad \xi^{1}=r
$$

leads to the induced metric:

$$
g_{\alpha \beta}=b^{2}(r)\left(\begin{array}{cc}
v^{2}-f(r) & v \xi^{\prime}(r) \\
v \xi^{\prime}(r) & f(r)^{-1}+\xi^{\prime 2}
\end{array}\right),
$$

and the corresponding action:

$$
S=-\frac{1}{2 \pi \ell_{s}^{2}} \int d t d r b^{2}(r) \sqrt{1-\frac{v^{2}}{f(r)}+f(r) \xi^{\prime 2}(r)} .
$$

Since $S$ does not depend on $\xi$ but only its derivative, the conjugate momentum $\pi_{\xi}$ is conserved,

$$
\pi_{\xi}=-\frac{1}{2 \pi \ell_{s}^{2}} \frac{b^{2}(r) f(r) \xi^{\prime}(r)}{\sqrt{1-\frac{v^{2}}{f(r)}+f(r) \xi^{\prime 2}(r)}}=-\frac{b^{2}\left(r_{s}\right) \sqrt{f\left(r_{s}\right)}}{2 \pi \ell_{s}^{2}}
$$

where the final expression is obtained by evaluating it at the point $r=r_{s}$, defined by

$$
f\left(r_{s}\right)=v^{2} .
$$

For an infinitely massive quark, the string endpoint is the boundary, $r=0$. For a quark of finite mass $M_{Q}$, the endpoint should be located at a position $r_{Q}$ in the interior, as discussed in detail in [15]. This puts an upper bound on the quark velocity $v$, since the trailing string picture fails when $r_{s}<r_{Q}$. At this point, the flavor brane dynamics should become important.

The Drag Force The drag force on the quark can be determined by calculating the momentum that is lost by flowing from the string to the horizon, which results in:

$$
F_{\text {drag }}=\pi_{\xi}=-\frac{v b^{2}\left(r_{s}\right)}{2 \pi \ell_{s}^{2}},
$$

where we have replaced $f\left(r_{s}\right)$ by $v^{2}$ in the last equality.

One defines the momentum friction coefficient $\eta_{D}$ as the characteristic attenuation constant for the momentum of a quark of mass $M_{q}$ :

$$
F_{\text {drag }}=\frac{d p}{d t} \equiv-\eta_{D} p, \quad, \quad p=M_{q} v \gamma
$$

where $\gamma=\left(1-v^{2}\right)^{-1 / 2}$ is the relativistic contraction factor. With this definition we obtain:

$$
\eta_{D}=\frac{1}{\gamma M_{q}} \frac{b^{2}\left(r_{s}\right)}{2 \pi \ell_{s}^{2}} .
$$

In the conformal case, $\eta_{D}$ is independent of $p$,

$$
\eta_{D}^{\mathrm{conf}}=\frac{\pi \sqrt{\lambda} T^{2}}{2 M_{q}},
$$

where $\lambda=\left(\ell / \ell_{s}\right)^{4}$ is the fixed 't Hooft coupling of $\mathcal{N}=4 \mathrm{sYM}$. This is not anymore so in the general case, where $\eta_{D}$ is momentum dependent. 
The world-sheet black hole. The coordinate value $r=r_{s}$ is a horizon for the induced world-sheet metric. In order to ascertain this, we can invert equation (3.14) to obtain $\xi^{\prime}(r)$ in the form:

$$
\xi^{\prime}(r)=\frac{C}{f(r)} \sqrt{\frac{f(r)-v^{2}}{b^{4}(r) f(r)-C^{2}}} \quad, \quad C \equiv v b^{2}\left(r_{s}\right) .
$$

We may now change coordinates to diagonalize the induced metric, by means of the reparametrization:

$$
t=\tau+\zeta(r), \quad \zeta^{\prime}=\frac{v \xi^{\prime}}{f-v^{2}}=\frac{C v}{f(r) \sqrt{\left(f(r)-v^{2}\right)\left(b^{4} f-C^{2}\right)}} .
$$

In these coordinates the induced metric is

$$
d s^{2}=b^{2}\left[-\left(f(r)-v^{2}\right) d \tau^{2}+\frac{b^{4}}{\left(b^{4} f-C^{2}\right)} d r^{2}\right] .
$$

The coefficient of $d \tau^{2}$ vanishes at $r_{s}$, so this point corresponds to a world-sheet black-hole horizon. Since $f(r)$ runs between 0 and 1 as $0<r<r_{h}$, by definition (3.15) the world-sheet horizon is always outside the bulk black hole horizon, and it coincides with it only in the limit $v \rightarrow 0$. In the opposite limit, $v \rightarrow 1, r_{s}$ asymptotes to the boundary $r=0$.

The Hawking temperature associated to the black hole metric (3.22) is found as usual, by expanding around $r=r_{s}$ and demanding regularity of the Euclidean geometry. The resulting temperature is:

$$
T_{s} \equiv \frac{1}{4 \pi} \sqrt{f\left(r_{s}\right) f^{\prime}\left(r_{s}\right)\left[\frac{4 b^{\prime}\left(r_{s}\right)}{b\left(r_{s}\right)}+\frac{f^{\prime}\left(r_{s}\right)}{f\left(r_{s}\right)}\right]} .
$$

In the conformal limit, where the dilaton is constant and the background solution reduces to AdS-Schwarzschild, the world-sheet temperature and horizon position are simply given by:

$$
T_{s}^{\text {conf }}=\frac{T}{\sqrt{\gamma}}, \quad r_{s}^{\text {conf }}=\frac{1}{\pi \sqrt{\gamma} T} .
$$

More generally, in the ultra-relativistic limit $v \simeq 1$, one can express $r_{s}$ in terms of thermodynamic quantities. In this limit $r_{s}$ approaches the boundary $r=0$, and in this region the geometry approaches that of AdS-Schwarzschild, equations (3.6), (3.7). Therefore, from the definition (3.15) we obtain:

$$
r_{s} \simeq \frac{1}{\sqrt{\gamma}}\left(\frac{4 N_{c}^{2}}{45 \pi^{2} s T}\right)^{\frac{1}{4}}, \quad v \rightarrow 1
$$

\subsection{Fluctuations of the trailing string}

We now proceed to study the quadratic fluctuations around the classical trailing string solution reviewed in the previous section. This analysis was performed in the AdS black hole background in [18]. Here, we extend it to the general 5D background (3.1). 
We continue to work in the static gauge $\xi^{0}=t, \xi^{1}=r$, but we will allow for a more general ansatz for the embedding coordinates:

$$
X^{1}=v t+\xi(r)+\delta X^{\|}(r, t), \quad X^{2}=\delta X^{2}(r, t) \quad, \quad X^{3}=\delta X^{3}(r, t) .
$$

We will treat the quantities $\delta X^{i}$ as perturbations around the background solution (3.10).

The Nambu-Goto action (3.8) is now given by:

$$
S_{\mathrm{NG}}=-\frac{1}{2 \pi \ell_{s}^{2}} \int d t d r \sqrt{\hat{g}_{r t}^{2}-\hat{g}_{t t} \hat{g}_{r r}}
$$

where

$$
\hat{g}_{t t}=b^{2}\left(-f+\dot{X}^{i} \dot{X}^{i}\right) \quad, \quad \hat{g}_{r r}=b^{2}\left(\frac{1}{f}+X^{i^{\prime}} X^{i^{\prime}}\right) \quad, \quad \hat{g}_{r t}=b^{2} X^{i^{\prime}} \dot{X}^{i}
$$

where a dot and a prime represent derivatives w.r.t. $t$ and $r$ respectively.

Expanding the Nambu-Goto action in $\delta X^{i}$ around the classical solution (3.20) we obtain, to quadratic order:

$$
S_{2}=-\frac{1}{2 \pi \ell_{s}^{2}} \int d t d r G^{\alpha \beta}\left[\frac{1}{2} \partial_{\alpha} \delta X^{\|} \partial_{\beta} \delta X^{\|}+\frac{Z^{2}}{2} \sum_{i=2}^{3} \partial_{\alpha} \delta X^{i} \partial_{\beta} \delta X^{i}\right],
$$

where

$$
G^{\alpha \beta}=\frac{b^{2}}{Z^{3}}\left(\begin{array}{cc}
-\frac{Z^{2} f+v^{2}}{f^{2}} & v \xi^{\prime} \\
v \xi^{\prime} & f-v^{2}
\end{array}\right)
$$

and we have defined:

$$
Z \equiv b^{2} \sqrt{\frac{f-v^{2}}{b^{4} f-C^{2}}} .
$$

Note that $\operatorname{det}\left(G^{\alpha \beta}\right)=-b^{4} / Z^{4}$ and that in the $\mathcal{N}=4$ case $Z=\sqrt{1-v^{2}}$ is a constant.

In terms of the induced world-sheet metric (3.12), we obtain

$$
G^{\alpha \beta}=Z^{-1} b^{4} g^{\alpha \beta}, \quad \sqrt{-\operatorname{det} g}=b^{2} Z .
$$

We may therefore rewrite the action as

$$
S_{2}=-\frac{1}{2 \pi \ell_{s}^{2}} \int d t d r \frac{b^{2}}{2} \sqrt{-\operatorname{det} g} g^{\alpha \beta}\left[\frac{1}{Z^{2}} \partial_{\alpha} \delta X^{\|} \partial_{\beta} \delta X^{\|}+\sum_{i=2}^{3} \partial_{\alpha} \delta X^{i} \partial_{\beta} \delta X^{i}\right]
$$

To simplify the action, we change coordinates to diagonalize the induced metric, as in the previous subsection. By a reparametrization of the world-sheet time coordinate as in (3.21), the new induced metric is (3.22), and the action read:

$$
S_{2}=-\frac{1}{2 \pi \ell_{s}^{2}} \int d \tau d r \frac{1}{2} H^{\alpha \beta}\left[\frac{1}{Z^{2}} \partial_{\alpha} \delta X^{\|} \partial_{\beta} \delta X^{\|}+\sum_{i=2}^{3} \partial_{\alpha} \delta X^{i} \partial_{\beta} \delta X^{i}\right]
$$

with

$$
H^{\alpha \beta}=\left(\begin{array}{cc}
-\frac{b^{4}}{\sqrt{\left(f-v^{2}\right)\left(b^{4} f-C^{2}\right)}} & 0 \\
0 & \sqrt{\left(f-v^{2}\right)\left(b^{4} f-C^{2}\right)}
\end{array}\right),
$$


Equations (3.29) and (3.34) show that the longitudinal fluctuations (i.e. those parallel to the direction of the unperturbed trailing string motion), namely $\delta X^{\|}$, and the transverse fluctuation $\delta X^{2}$ and $\delta X^{3}$, have different kinetic terms, as the effective two-dimensional metrics they are sensitive to differ by a factor $Z^{2}$. From now on, we will denote the longitudinal fluctuations as $\delta X^{\|}$and the transverse fluctuations as $\delta X^{\perp}$.

From equation (3.22) one can immediately derive the field equations satisfied by the fluctuations:

$$
\partial_{\alpha} Z^{-2} H^{\alpha \beta} \partial_{\beta} \delta X^{\|}=0 \quad, \quad \partial_{\alpha} H^{\alpha \beta} \partial_{\beta} \delta X^{\perp}=0 .
$$

For a harmonic ansatz of the form $\delta X^{i}(r, \tau)=e^{i \omega \tau} \delta X^{i}(r, \omega)$, equations (3.36) become:

$$
\begin{aligned}
& \partial_{r}\left[\sqrt{\left(f-v^{2}\right)\left(b^{4} f-C^{2}\right)} \partial_{r}\left(\delta X^{\perp}\right)\right]+\frac{\omega^{2} b^{4}}{\sqrt{\left(f-v^{2}\right)\left(b^{4} f-C^{2}\right)}} \delta X^{\perp}=0 \\
& \partial_{r}\left[\frac{1}{Z^{2}} \sqrt{\left(f-v^{2}\right)\left(b^{4} f-C^{2}\right)} \partial_{r}\left(\delta X^{\|}\right)\right]+\frac{\omega^{2} b^{4}}{Z^{2} \sqrt{\left(f-v^{2}\right)\left(b^{4} f-C^{2}\right)}} \delta X^{\|}=0
\end{aligned}
$$

In the next sections we will compute the Langevin correlation functions from these fluctuation equations and extract the diffusion constants and the spectral densities from them.

We note however that the diffusion constants can also be read-off directly from the quadratic action (3.34) by using the method of the membrane paradigm as explained in section 4.2 .

\section{Holographic computation of Langevin correlators}

\subsection{The Green's functions}

From the discussion in the previous section, it emerges that in a $4 \mathrm{D}$ theory with a $5 \mathrm{D}$ gravity dual we can compute the Langevin correlators holographically, from the classical solutions for the fluctuations of the trailing string. As we have observed in section 3, these fluctuations behave as free fields propagating on a 2D black-hole background, whose metric is essentially the induced metric on the bulk trailing string, equation (3.22). The asymptotic form and the causal structure of this black hole are exactly the same as the one for the trailing string embedded in an AdS black hole. As a consequence, the results of $[18,19,22]$ discussed in the previous sections immediately generalize to the more general metric (3.1): following the Keldysh contour in the extended Kruskal diagram of the black hole, one finds a thermal spectrum of transverse and longitudinal fluctuations with effective temperature $T_{s}$, given in equation (3.23).

In this ensemble, the symmetrized and retarded Green's functions obey the relation (2.14), with $T=T_{s}$. Therefore, one can obtain both the memory kernel and the noise correlator entering equations (2.2)-(2.3) from the knowledge of the retarded Green's function.

From the structure of the action for the fluctuations, equation (3.33), one can observe that there are essentially two types of retarded correlators, $G_{R}^{\|}(\omega)$ and $G_{R}^{\perp}(\omega)$, for the 
longitudinal and transverse fluctuations. We introduce a notation similar to [18] and define

$$
\mathcal{G}_{\perp}^{\alpha \beta} \equiv \frac{1}{2 \pi \ell_{s}^{2}} H^{\alpha \beta}, \quad \mathcal{G}_{\|}^{\alpha \beta} \equiv \frac{1}{2 \pi \ell_{s}^{2}} \frac{H^{\alpha \beta}}{Z^{2}}
$$

where $H^{\alpha \beta}$ is defined in equation (3.35).

The holographic prescription for the retarded correlator, computed with the diagonal induced metric (3.22) is given by:

$$
G_{R}(\omega)=-\left[\Psi_{R}^{*}(r, \omega) \mathcal{G}^{r r} \partial_{r} \Psi_{R}(r, \omega)\right]_{\text {boundary }} .
$$

Here $\Psi_{R}(r, \omega)$ denotes collectively the fluctuations $\delta X^{\|}, \delta X^{\perp}$, solutions of equations (3.37)-(3.38) with the appropriate boundary conditions, i.e. unit normalization at the boundary, and infalling conditions at the world-sheet horizon (as we discuss more extensively below) and the factor $\mathcal{G}^{\text {rr }}$ is the appropriate one from equation (4.1).

The expression in equation (4.2) must be evaluated at the boundary of the trailing string world-sheet. In the case of an infinitely massive quark, the string is attached at the AdS boundary at $r=0$ (when needed, in order to keep quantities finite, we introduce a cut-off boundary at $r=\epsilon$ ). In case we want to keep the quark mass finite, the trailing string is attached to a point $r_{Q}$, which is determined by demanding that the free energy of a static string in the $T=0$ background gives the mass of the quark:

$$
M_{q}=\frac{1}{2 \pi \ell_{s}^{2}} \int_{r_{Q}}^{r_{*}} b(r)^{2} d r
$$

(here $r_{*}$ is the point at which $b(r)$ reaches its minimum, see the discussion in [15]).

Next, we discuss in greater detail the boundary conditions for the $\Psi_{R}$ 's. The solutions to the fluctuation equations (3.37)-(3.38) share the same asymptotics both for the transverse and longitudinal components (since $Z(r)$ asymptotes to a constant both at the horizon and at the boundary, where the equations have singular points). At the world-sheet horizon $r \rightarrow r_{s}$ equations (3.37)-(3.38) both take the form

$$
\partial_{r}^{2} \Psi+\frac{1}{\left|r-r_{s}\right|} \partial_{r} \Psi+\left(\frac{\omega}{4 \pi T_{s}\left|r-r_{s}\right|}\right)^{2} \delta \Psi=0
$$

so that the solutions near the horizon behave as

$$
\Psi(r, \omega) \sim\left(r_{s}-r\right)^{ \pm \frac{i \omega}{4 \pi T_{s}}}+\cdots
$$

The + sign in the exponent corresponds to a wave which is outgoing with respect to the world-sheet horizon, while the - sign characterizes an in-falling wave.

Near the boundary $r \rightarrow 0$ both transverse and longitudinal fluctuations have to solve the following equation:

$$
\partial_{r}^{2} \Psi(r, \omega)-\left(\frac{2}{r}-\frac{4}{3} \frac{\lambda^{\prime}}{\lambda}\right) \partial_{r} \Psi(r, \omega)+\gamma^{2} \omega^{2} \Psi(r, \omega)=0
$$


As long as $r \lambda^{\prime} / \lambda \ll 1,{ }^{9}$ the two independent solutions are a normalizable mode and a non-normalizable mode,

$$
\Psi \sim C_{s}+C_{v} r^{3} \lambda^{-4 / 3}
$$

According to the standard prescription [57], the appropriate boundary conditions for the wave functions $\Psi_{R}$ in the expression (4.2) for the retarded correlator are the in-falling behavior at the world-sheet horizon with the condition $\Psi_{R}(r)=1$ at the boundary:

$$
\begin{aligned}
& \Psi_{R}\left(r_{b}, \omega\right)=1 \quad r_{b}=\left\{\begin{array}{cc}
0 & M_{q} \rightarrow \infty \\
r_{Q} & M_{q} \text { finite }
\end{array}\right. \\
& \Psi_{R}(r, \omega) \simeq \Psi_{h}\left(r_{s}-r\right)^{-\frac{i \omega}{4 \pi T_{s}}} \quad r \sim r_{s} .
\end{aligned}
$$

where $\Psi_{h}$ is a constant.

Given the wave-function, obeying the near-boundary and near-horizon asymptotics specified by equations (4.8)-(4.9), we can extract the propagator from equation. (4.2). Below, we separately discuss the features of the real and imaginary parts of the retarded Green's functions, and the associated spectral densities.

Real part of the retarded correlators. The real part of the correlator (4.2) suffers from ambiguities related to the possibility of adding boundary counterterms to the action (3.8). This was discussed e.g. in [58] in the context of the calculation of 4-dimensional transport coefficients.

The ambiguities in the propagator are, as usual, associated to UV-divergences in the on-shell action, that arise when we try to evaluate it in the full AdS space-time. To obtain a finite result, we must consider the action on a regularized space-time, with boundary at $r=\epsilon$ rather than $r=0$. Then, once the divergences in the limit $\epsilon \rightarrow 0$ are identified, one can add counterterms to subtract them and obtain a finite limit as $\epsilon \rightarrow 0$.

The essence of holographic renormalization is that these counterterms are local, covariant boundary terms. As we show in appendix A, we only need a single boundary counterterm to regularize the action, and this is given by the boundary-covariant point-particle action:

$$
S_{\text {count }}=\Delta M(\epsilon) \int d t \sqrt{\dot{X}^{\mu} \dot{X}_{\mu}}
$$

The same UV divergences appear in the real part of the propagator, if we try to compute it naively from equation (4.2). In fact, the expression (4.2) is nothing but the unrenormalized on-shell action, as can be easily observed by integrating equation (3.34) by parts and using the field equations (3.36). Therefore, as a consequence of the analysis of the on-shell action carried out in appendix A the divergent parts of the transverse and longitudinal Green's functions are:

$$
\left(\operatorname{Re} G_{R}^{\perp}\right)^{(\mathrm{div})}=\frac{\lambda^{4 / 3}(\epsilon)}{\epsilon} \frac{\ell^{2}}{2 \pi \ell_{s}^{2}} \gamma \omega^{2}, \quad\left(\operatorname{Re} G_{R}^{\|}\right)^{(\mathrm{div})}=\frac{\lambda^{4 / 3}(\epsilon)}{\epsilon} \frac{\ell^{2}}{2 \pi \ell_{s}^{2}} \gamma^{3} \omega^{2} .
$$

\footnotetext{
${ }^{9}$ The condition $r \lambda^{\prime} / \lambda \ll 1$ is realized in particular in the case of logarithmic running: in that case, for small $r, r \lambda^{\prime} / \lambda \sim \lambda \ll 1$. In the case where $\lambda$ is dual to a relevant operator it is also valid a fortiori since $r \lambda^{\prime} / \lambda \sim \tilde{\Delta} r$, with $\tilde{\Delta}=\min (\Delta, 4-\Delta)$.
} 
This result can also be explicitly derived from the explicit form of the wave-functions, close to the boundary. As we will show in subsection 4.4, (see appendix $\mathrm{C}$ for a more detailed derivation) the solution of eq. (4.6) is,

$$
\Psi_{\mathrm{UV}}(r)=[\cos (\gamma \omega r)+(\gamma \omega r) \sin (\gamma \omega r)]+C_{v} \lambda^{-4 / 3}(r)[(\gamma \omega r) \cos (\gamma \omega r)-\sin (\gamma \omega r)]
$$

This solution generalizes eq. (4.7) for any finite $\omega$, and it is valid in the near-boundary region, i.e. for $\gamma \omega r \ll 1$, and $\lambda(r) \ll 1$. The value of the coefficient $C_{s}$ of the leading term is fixed to ensure unit normalization at $r=\epsilon$ (we are keeping in mind that we will take the $\epsilon \rightarrow 0$ limit at the end).

Evaluating the real part of (4.2) at $r=\epsilon$ with the wave-function given by (4.12), we find the following divergent term for the transverse and longitudinal components:

$$
\operatorname{Re} G_{R}^{\perp} \simeq \gamma^{-2} \operatorname{Re} G_{R}^{\|} \simeq \frac{\lambda^{4 / 3}(\epsilon)}{\epsilon} \gamma \omega^{2}\left[1+\frac{1}{2} \epsilon^{2} \gamma^{2} \omega^{2}+O\left(\epsilon^{4}\right)\right] \frac{\ell^{2}}{2 \pi \ell_{s}^{2}}, \quad \epsilon \rightarrow 0
$$

The divergence is purely in the $\omega^{2}$ term, and the coefficient agrees with the result we found from the on-shell action (4.11). Notice that the second term in equation (4.12), proportional to $C_{v}$, starts at $O\left(\epsilon^{3} \omega^{3}\right)$, so it does not contribute to the divergent part of the propagator.

To eliminate the divergence, and obtain a finite result, we must add the contribution from the boundary counterterm. However, different results can arise due to different choices for the finite contributions included in the counterterm, i.e. different subtraction schemes. As discussed in appendix A, in our case this ambiguity reduces to a term of the form $\delta G_{R}(\omega)=\delta m \omega^{2}$, which can be reabsorbed in the renormalization of the quark mass. We are going to use a minimal scheme, and fix the coefficient of the counterterm action (4.10) to be:

$$
\Delta M(\epsilon)=-\frac{\lambda^{4 / 3}(\epsilon)}{\epsilon} \frac{\ell^{2}}{2 \pi \ell_{s}^{2}} .
$$

This choice exactly subtracts the divergences (4.11) (see appendix A), and moreover removes all (finite or infinite) $\mathrm{O}\left(\omega^{2}\right)$ terms in the large- $\omega$ behavior of the propagator.

Once we subtract the divergence in the minimal scheme, and we take the limit $\epsilon \rightarrow 0$, the right hand side of equation (4.13) vanishes. This means that, in this scheme, $\operatorname{Re} G_{R}(\omega) \rightarrow 0$ as $\omega \rightarrow \infty$. Moreover, as the only ambiguity in $\operatorname{Re} G_{R}$ is proportional to $\omega^{2}$ (see appendix A), we conclude that in any other scheme the real part of the Langevin Green's function grows as $\omega^{2}$ for large $\omega$.

As a final remark, the previous discussion only applies if we consider the quark mass to be infinite. In the case of a finite mass, the trailing string is attached at a radial point $r_{Q}>$ 0 , the cut-off is physical, and the result is not divergent. However, one should still specify the finite boundary term included in the action in order to arrive at an unambiguous result.

Imaginary part, and the symmetric correlators. Unlike the real part, the imaginary part of the retarded correlator does not suffer from ambiguities. One of the reasons is that it is proportional to a conserved quantity, which can be shown to be finite at the horizon. In fact, we can write $\operatorname{Im} G_{R}$ in the form:

$$
\operatorname{Im} G_{R}(\omega)=-\frac{1}{2 i} \mathcal{G}^{r r} \Psi_{R}^{*} \overleftrightarrow{\partial_{r}} \Psi_{R} \equiv-J^{r}
$$


Here $J^{r}$ is a conserved current - this follows directly from the equations for the fluctuations, equations (3.36) - hence the imaginary part of the retarded correlator can be analytically evaluated at any $r$, not necessarily at the boundary. It is convenient to evaluate it at the horizon. From the definitions (4.1) and (3.35), we find that, in the near-horizon limit:

$$
\mathcal{G}_{\perp}^{r r} \simeq Z^{2}\left(r_{s}\right) \mathcal{G}_{\|}^{r r} \simeq\left(4 \pi T_{s}\right) b\left(r_{s}\right)^{2}\left(r_{s}-r\right), \quad r \rightarrow r_{s}
$$

Inserting this expression in equation (4.15), and using the expression (4.9) for $\Psi(r)$, we find:

$$
\operatorname{Im} G_{R}^{\perp}(\omega)=-\frac{b^{2}\left(r_{s}\right)}{2 \pi \ell_{s}^{2}}\left|\Psi_{h}^{\perp}(\omega)\right|^{2} \omega, \quad \operatorname{Im} G_{R}^{\|}(\omega)=-\frac{b^{2}\left(r_{s}\right)}{2 \pi \ell_{s}^{2} Z^{2}\left(r_{s}\right)}\left|\Psi_{h}^{\|}(\omega)\right|^{2} \omega,
$$

where $\Psi_{h}$ is the coefficient of the in-falling wave-function, see equation (4.9).

From the imaginary part of $G_{R}(\omega)$ we can immediately extract the symmetrized correlator $G_{\text {sym }}(\omega)$, i.e. the generalized Langevin noise time-correlation function: as discussed at the beginning of this section, due to the thermal nature of the world-sheet fluctuations, $G_{\text {sym }}(\omega)$ is related to $\operatorname{Im} G_{R}(\omega)$ by the following equation, which generalizes the analogous equations in $[18,19,22]$ :

$$
G_{\mathrm{sym}}(\omega)=-\operatorname{coth}\left(\frac{\omega}{2 T_{s}}\right) \operatorname{Im} G_{R}(\omega)
$$

The spectral densities. The spectral densities associated with the Langevin dynamics are defined by

$$
\begin{aligned}
\rho^{a}(\omega) & =-\frac{1}{\pi} \operatorname{Im} G_{R}^{a}(\omega), \\
\rho_{\mathrm{sym}}^{a}(\omega) & =-\frac{1}{\pi} G_{\mathrm{sym}}^{a}(\omega)=-\operatorname{coth}\left(\frac{\omega}{2 T_{s}}\right) \rho^{a}(\omega),
\end{aligned}
$$

where $a=\perp, \|$, and we have used equation (4.18) in the second line. The imaginary part of $G_{R}$ is given by the flux for the perpendicular and the parallel components by the formula (4.15). We will give an analytical estimation of the large-frequency behavior of the spectral densities in subsection 4.4, using a WKB method; in section 6 we will use numerical methods to obtain the full functional dependence of $\rho(\omega)$ in a concrete model.

\subsection{The membrane paradigm}

Here we introduce an alternative method to calculate both the diffusion constants and the spectral densities that goes under the name of the membrane paradigm [51]. We apply this method to obtain the spectral densities in the next section and to obtain the diffusion constants, in section 5.3.

In [51], it was established that the transport coefficients associated with generic massless fluctuations can be read off directly from their effective coupling in the action, evaluated at the horizon. For an arbitrary massless fluctuation $\phi$ with an action

$$
S_{2}=-\frac{1}{2} \int d^{d} x d r \sqrt{-\operatorname{det} g} Q(r) g^{\alpha \beta} \partial_{\alpha} \phi \partial_{\beta} \phi,
$$


the transport coefficient associated with the retarded Green's function is given by,

$$
\chi_{R}=-\lim _{k_{\mu} \rightarrow 0} \frac{\operatorname{Im} G_{R}(\omega, \vec{k})}{\omega}=\left.\lim _{k_{\mu} \rightarrow 0} Q\left(r_{s}\right) \sqrt{\frac{-\operatorname{det} g}{g_{r r} g_{t t}}}\right|_{r_{s}}
$$

where $Q$ is the effective coupling of the fluctuation defined in (4.21). We refer the reader to [51] for a derivation of this formula, noting that here we apply the same idea to the world-sheet black-hole rather than the bulk black-hole as in [51].

In our case, comparison of (4.21) with (3.33) yields,

$$
Q_{\|}=\frac{1}{\pi \ell_{s}^{2}} \frac{b^{2}}{Z^{2}}, \quad Q_{\perp}=\frac{1}{\pi \ell_{s}^{2}} b^{2} .
$$

Once we know these effective couplings, we can immediately write down the diffusion constants. Therefore, the method provides a very efficient and fast way of computing the latter. This is done in section 5.3.

\subsubsection{A differential equation for spectral densities}

Although the method of the membrane paradigm is most effective in the low frequency limit, where one can read off transport coefficients directly from the $Q$ 's, it is still a convenient method for arbitrary $\omega$ where one has to use the flow equations [51]. We define the canonical momentum associated with $\phi$ in (4.21) with respect to foliations in $r$, as

$$
\Pi(r, \omega, \vec{k})=Q(r) \sqrt{-\operatorname{det} g} g^{r r} \partial_{r} \phi(r, \omega, \vec{k}),
$$

where we performed the Fourier transform on the $4 \mathrm{D}$ space-time. We also define the " $r$ dependent" response function

$$
\bar{\chi}=i \frac{\Pi(x, \omega, \vec{k})}{\omega \phi(r, \omega, \vec{k})} .
$$

Using the general AdS/CFT relation for the retarded Green function,

$$
G_{R}(\omega, \vec{k})=\lim _{r \rightarrow 0} \frac{\Pi(x, \omega, \vec{k})}{\phi(r, \omega, \vec{k})},
$$

we find that,

$$
\rho(\omega, \vec{k})=-\frac{1}{\pi} \operatorname{Im} G_{R}(\omega, \vec{k})=\frac{\omega}{\pi} \lim _{r \rightarrow 0} \operatorname{Re} \bar{\chi} .
$$

From the equations of motion, one derives a first-order equation for $\bar{\chi},[51]:^{10}$

$$
\partial_{r} \bar{\chi}=i \omega \sqrt{\frac{g_{r r}}{g_{t t}}}\left(\frac{1}{Q(r)} \bar{\chi}^{2}-Q(r)\right) .
$$

Here, the effective coupling $Q$ is given by (4.23) and,

$$
\sqrt{\frac{g_{r r}}{g_{t t}}}=\frac{Z(r)}{f(r)-v^{2}} .
$$

\footnotetext{
${ }^{10}$ In the rest of this section, we set $\vec{k}=0$.
} 
For regularity at $r=r_{s}$ one should require that $\bar{\chi} \rightarrow Q\left(r_{s}\right)$ as $r \rightarrow r_{s}$. One solves (4.28) with this boundary condition at the horizon, and determines $\bar{\chi}$ on the boundary $r=r_{b}$. The spectral density associated with the symmetric Green's function is given by,

$$
\rho_{\mathrm{sym}}(\omega)=\frac{\omega}{\pi} \operatorname{coth}\left(\frac{\omega}{2 T_{s}}\right) \operatorname{Re} \bar{\chi}\left(r_{b}, \omega\right) .
$$

As we show in section $4.4, \rho_{\text {sym }}$ is divergent as $\omega \rightarrow \infty$. This is similar to the familiar short-distance divergences of correlators of quantum field theory.

\subsection{Universal results for the spectral densities}

The membrane paradigm allows us to obtain interesting relations concerning the spectral densities in certain limits. We first note that, by employing the equation of motion for $\phi$ that follows from (4.21) and (4.28) one can show that $|\phi|^{2} \operatorname{Re} \bar{\chi}$ is independent of $r$ :

$$
|\phi(r)|^{2} \operatorname{Re} \bar{\chi}(r)=N(\omega) .
$$

Through equation (4.27), this is of course equivalent to the fact that the imaginary part of the Green function is proportional to the conserved flux, hence it is constant.

We may evaluate (4.31) in the two limits $r=r_{b}$ and $r=r_{s}$. We first evaluate it at the horizon: the in-falling condition is,

$$
\phi(r, \omega) \rightarrow C_{h}\left(r_{s}, \omega\right)\left(1-r / r_{s}\right)^{-\frac{i \omega}{4 \pi T_{s}}}, \quad r \rightarrow r_{s} .
$$

Therefore, $\left|\phi\left(r_{s}\right)\right|^{2}=\left|C_{h}\right|^{2}$. On the other hand, by the boundary condition of (4.28), $\bar{\chi}\left(r_{s}\right)=Q\left(r_{s}\right)$. Therefore we obtain, $N(\omega)=C_{h}\left(\omega, r_{s}\right) Q\left(r_{s}\right)$.

Secondly, we evaluate (4.31) at the boundary $r_{b}$ using $(4.27)$ and we obtain $N(\omega)=$ $\pi \rho(\omega) / \omega$. Hence we have,

$$
\rho(\omega)=\frac{\omega}{\pi} Q\left(r_{s}\right)\left|C_{h}\right|^{2}\left(r_{s}, \omega\right) .
$$

Now, we consider the special limit $r_{s} \rightarrow r_{b}$ while keeping $\omega$ finite, or more precisely we consider $\omega r_{s} \ll 1$. If we think of $\omega$ as being fixed, this limit can be attained in two ways:

- either by sending $T \rightarrow \infty$, so that the black hole horizon, and consequently the world-sheet horizon, are pushed to the AdS boundary;

- or by keeping $T$ finite, and sending $v \rightarrow 1$. In this case the black hole temperature is fixed, but the string is ultra-relativistic.

Therefore, the regime $\omega r_{s} \ll 1$ corresponds to a UV limit for the background quantities $T$ and $v$.

On the other hand, for a given fixed $\omega$, as $r_{s}$ approaches the boundary $C_{h}(\omega)$ becomes independent of $\omega$ and approaches unity. This is because of the unit normalization of $\phi$ at the boundary, $\phi\left(r_{b}\right)=1$. Therefore, (4.33) simplifies and one obtains,

$$
\rho(\omega) \simeq \frac{\omega}{\pi} Q\left(r_{s}\right), \quad \omega r_{s} \ll 1
$$


We can make this expression more explicit by taking the near-boundary approximate expressions, valid for $r_{s} \sim 0$, in (4.23): we approximate the metric functions as in equation (3.6), and consequently, from equation (3.31), $Z\left(r_{s}\right) \simeq \gamma^{-1}$. These approximations are valid up to terms of $\mathcal{O}(\lambda)$, which signal the departure from conformality of the UV geometry. Using these approximations we find:

$$
Q_{\perp}\left(r_{s}\right) \simeq \gamma^{-2} Q_{\|}\left(r_{s}\right) \simeq \frac{\ell^{2}}{\ell_{s}^{2}} \frac{\lambda^{\frac{4}{3}}\left(r_{s}\right)}{\pi r_{s}^{2}}\left[1+\mathcal{O}\left(\lambda\left(r_{s}\right)\right)\right]
$$

Finally, $r_{s}$ can also be expressed purely in terms of the boundary quantities in the same limit, using the near-boundary approximation (3.25).

The conclusion of the previous discussion is that, in the high energy limit one obtains universal expressions for the spectral densities, where they become linear in frequency.

This universal behavior is to be expected based on the consideration that, for fixed $\omega$, both limits $v \rightarrow 1$ and $T \rightarrow \infty$ correspond to the limit $r_{s} \rightarrow 0$. In this regime the equation governing the string fluctuation become essentially the equation one finds in pure AdS, close to the boundary. This case is discussed explicitly in appendix D, from which one concludes (see equation (D.1)) that the wave-functions depend on $\omega$ and $T_{s}$ only through the combination $\omega / T_{s} \propto \omega r_{s}$. Thus, for fixed $\omega$ and small $r_{s}$, i.e. for $\omega r_{s} \ll 1$, the spectral density is approximated by the linear term in the expansion in $\omega r_{s}$.

\subsection{The WKB approximation at large frequency}

The WKB approximation can be used to obtain the high-frequency behavior of the Langevin spectral densities. Here we summarize the method and present the results. The detailed derivation is given in appendix $\mathrm{C}$.

By a rescaling of the wave-function, the fluctuation equations (3.37)-(3.38) can be put in a Schrödinger-like form, and the large $\omega$ solution can be obtained by an adaptation of the WKB method. This method has been applied to the case of shear perturbations in AdS-Schwarzschild black-hole in [52].

The fluctuation equations (3.37)-(3.38) can be put in the Schrödinger form

$$
-\psi^{\prime \prime}+V_{s}(r) \psi=0, \quad V_{s}(r)=-\frac{\omega^{2} b^{4}}{R^{2}}+\frac{1}{2}(\log \mathcal{R})^{\prime \prime}+\frac{1}{4}(\log \mathcal{R})^{\prime 2} .
$$

defining the wave function $\psi=\mathcal{R}^{\frac{1}{2}} \Psi$ and $R=\sqrt{\left(f-v^{2}\right)\left(b^{4} f-C^{2}\right)}$, where

$$
\Psi=\left\{\begin{array}{l}
\delta X^{\perp} \\
\delta X^{\|}
\end{array}, \quad \mathcal{R}=\left\{\begin{array}{l}
R \\
R / Z^{2}
\end{array}\right.\right.
$$

For large frequency (compared to $r_{s}^{-1}$ ), we can approximate the potential over essentially the entire range of $r$ by the expression:

$$
V_{s}(r) \simeq-\frac{\omega^{2} b^{4}}{R^{2}}, \quad \omega r_{s} \gg 1, \quad r_{t p} \ll r<r_{s},
$$

where $r_{t p}$ denotes the classical turning point, $V\left(r_{t p}\right)=0$. The range of $r$ for which equation (4.38) is valid is the classically allowed region. For large $\omega r_{s}$, the turning point is 
approximately $r_{t p} \simeq \sqrt{2} /(\gamma \omega) \ll r_{s}$. Therefore, the classically allowed region covers almost all the range $0<r<r_{s}$, but for a small region close to the boundary (that includes the turning point) where the approximation (4.38) breaks down. Finally, and most importantly, for $\omega r_{s} \gg 1$ there is always an overlap region in which the boundary asymptotics of the Schrödinger equation and equation (4.38) are both valid, since $r_{t p} \ll r_{s}$. This will allow the matching of the WKB solution and the boundary solution.

In order to obtain a large- $\omega$ analytic approximation for $\rho(\omega)$, it is necessary to specify whether we consider the quark mass as infinite, or we are working with a finite but large mass $M_{q}$.

Infinitely massive quarks. In this case the endpoint of the string is attached to the AdS boundary $r=0$, and this is where we should set the normalization of the wave-functions.

The WKB computation proceeds along the steps detailed in appendix C. The WKB solution is matched with the horizon and boundary asymptotics, for the wave function, determining the coefficients $\Psi_{h}$ appearing in equation (4.17)

$$
\Psi_{h}(\omega)=\omega \frac{i \ell \gamma \lambda_{t p}^{2 / 3}}{b\left(r_{s}\right)}\left\{\begin{array}{ll}
1, & \perp \\
\gamma Z\left(r_{s}\right), & \|
\end{array} .\right.
$$

Inserting these expressions in equation (4.17) and using the definition (4.19), we determine $\rho(\omega)$, in the limit $\omega \gg 1 / r_{s}$ :

$$
\rho_{\perp}(\omega) \simeq \gamma^{-2} \rho_{\|}(\omega) \simeq \frac{\omega^{3}}{2 \pi^{2}} \frac{\ell^{2}}{\ell_{s}^{2}} \gamma^{2} \lambda_{t p}^{\frac{4}{3}}(\omega)
$$

Here $\lambda_{t p}=\lambda\left(r_{t p}\right)$ where $r_{t p} \simeq \sqrt{2} /(\omega \gamma)$ is the classical turning point, as discussed in appendix C. For very large $\omega$, the dilaton profile is can be approximated as in equation (3.6),

$$
\lambda_{t p} \simeq b_{0}^{-1} \log ^{-1}\left[\frac{\omega \gamma}{\sqrt{2} \Lambda}\right]
$$

Finite mass quarks. The computation for the finite mass quarks follows the same steps as for the infinitely massive case, except that the boundary normalization of the wave function $\Psi(r)=1$ has to be imposed at the cutoff $r=r_{Q}$ (determined by equation (4.3)), rather than at the proper boundary $r=0$. As $M_{Q}$ becomes large (with respect to the UV scale $\Lambda), r_{Q} \sim 1 / M_{Q}$.

The presence of a finite cutoff at $r_{Q}$ implies some subtleties in the matching of the WKB solution with the boundary solution, as explained in appendix C.

To give an explicit result, we must distinguish two regimes which, for fixed $\omega / M_{q}$, correspond to small and large velocities.

- Small velocities. If $r_{Q} \ll 1 / \gamma \omega$ the finite mass spectral densities at large frequencies behave like the infinite mass ones, except for $\left(\gamma \omega r_{Q}\right)^{2}$ corrections. More explicitly, 
we obtain

$$
\begin{aligned}
\rho_{\perp}(\omega) \simeq & \gamma^{-2} \rho_{\|}(\omega) \\
\simeq & \frac{\omega^{3}}{\pi^{2}} \frac{\ell^{2}}{\ell_{s}^{2}} \gamma^{2} \lambda_{t p}^{\frac{4}{3}}(\omega)\left[1+\left(\gamma \omega r_{Q}\right)^{2}+\left[\left(\frac{\ell^{2} \lambda_{t p}^{\frac{4}{3}}}{\gamma r_{Q}^{3}} \int^{r_{Q}} \frac{d r^{\prime}}{\mathcal{R}\left(r^{\prime}\right)}\right)^{2}-1\right]\right. \\
& \left.\cdot\left(\sin \left(\gamma \omega r_{Q}\right)-\gamma \omega r_{Q} \cos \left(\gamma \omega r_{Q}\right)\right)^{2}\right]^{-1} .
\end{aligned}
$$

These expressions are valid in the regime where $\omega r_{s} \gg 1$, but at the same time $\gamma \omega r_{Q}<1$, i.e. for small velocities, given a fixed (large) quark mass and a given frequency. On the other hand, for fixed frequency and velocity but for $M_{q} \rightarrow \infty$, $r_{Q} \rightarrow 0$, the r.h.s. asymptotes to unity, and we recover the infinite quark mass expressions (4.40).

- Large velocities. Analogously, in the limit where $r_{Q} \gg r_{t p} \simeq \sqrt{2} / \gamma \omega$, the spectral functions read

$$
\rho_{\perp}(\omega) \simeq \gamma^{-2} \rho_{\|} \simeq \frac{\omega^{3}}{\pi^{2} \ell_{s}^{2}} \gamma^{3} r_{Q}^{2} \mathcal{R}_{Q}\left[1+\left(\gamma \omega r_{Q}\right)^{2}\right]^{-1}
$$

Hence, the difference with respect to the infinite mass result in this case is that $\lambda_{t p}^{4 / 3}$ which is $\omega$-dependent —is replaced by $\lambda_{Q}^{4 / 3}$ — which, instead, is $\omega$-independent.

We note that the large $\omega$ behavior for finite mass, both in the large and in the small cutoff regimes, changes with respect to the infinite mass case and becomes linear rather than cubic, due to the extra $\left(\gamma \omega r_{Q}\right)^{2}$ term in equations (4.42)-(4.43). This extra term comes from the fact that the solution has a subleading linear dependence on $r$, which is negligible in the infinite mass case, but enters the expression of the spectral function, $\rho \sim \Psi^{*} \Psi^{\prime}$, in the finite mass case, giving it a quadratic dependence on $r_{Q}$.

\section{Langevin diffusion constants}

The correlators and spectral densities discussed in the previous section are the building blocks of the generalized Langevin equation, (2.2). Now, we will focus on the long-time limit, discussed in section 2, in which equation (2.2) reduces to the local form (2.12), in which only the $\omega$-independent friction and diffusion coefficients, $\eta$ and $\kappa$, appear. They are given in equation (2.13) in terms of the zero-frequency limit of the Langevin Green's functions.

Therefore, in this section we consider the zero-frequency limit of the Green's functions constructed in section 4 . This will allow us to give the analytic results for the diffusion constants, both from the direct evaluation of the correlators, and using the membrane paradigm. 


\subsection{Diffusion constants via the retarded correlator}

The diffusion constant is defined in terms of the symmetric correlator $G_{\text {sym }}$ (see equation (2.13):

$$
\kappa=\lim _{\omega \rightarrow 0} G_{\text {sym }}=-2 T_{s} \lim _{\omega \rightarrow 0} \frac{\operatorname{Im} G_{R}(\omega)}{\omega},
$$

where in the second equality we have used the $\omega \rightarrow 0$ limit of equation (4.18).

The small frequency limit of the symmetric correlator can be evaluated analytically since we can determine the boundary-to-bulk wave function in this limit and discard higher orders in the evaluation of (5.1). More precisely, we write the small frequency limit of the horizon asymptotics of the $\Psi_{R}$ 's. Given the in-falling boundary condition (4.9) we obtain

$$
\Psi_{R}(r, \omega)=\Psi_{h}\left(r_{s}-r\right)^{-\frac{i \omega}{4 \pi T_{s}}} \simeq \Psi_{h}\left[1-\frac{i \omega}{4 \pi T_{s}} \log \left|r-r_{s}\right|+\ldots\right]
$$

This solution can be connected to the boundary asymptotics by the exact solution of the fluctuation equations (3.37)-(3.38) at $\omega=0$ which reads $\Psi_{R}(r, 0) \equiv 1$ once we impose the appropriate boundary condition $\Psi_{R}\left(r_{b}\right)=1$ (see appendix B for details). On the other hand, equation (5.2) reduces to $\Psi_{R}=\Psi_{h}$ in the strict $\omega=0$ limit. Therefore, the nearhorizon solution at small frequencies is entirely determined by equation (5.2) and the match to the boundary solution which yields $\Psi_{h}=1$ (both for transverse and longitudinal modes).

Furthermore, expanding for $\omega \ll 1$, we may also show (see appendix B) that the solution for all values of $r$ and small frequencies is given by

$$
\Psi_{R}=\left(r_{s}-r\right)^{-\frac{i \omega}{4 \pi T_{s}}}[1+\mathcal{O}(\omega)] .
$$

Now we may substitute the solution (5.3) in the expression for $\kappa$, using (5.1) and evaluating the current at the horizon.

Infinitely Massive Quarks. For infinite mass quarks, the boundary is located at $r=0$ and the appropriate boundary-to-bulk wave function $\Psi_{R}$ is given by equation (5.3) at small frequencies.

To compute the diffusion constants (5.1), we evaluate $J^{r}$, defined in equation (4.15), at the radius value $r=r_{s}-\epsilon$ and then let $\epsilon \rightarrow 0$ (since $J^{r}$ is conserved it can be evaluated at any radius and not necessarily at the boundary, where subleading $\mathcal{O}(\omega)$ terms in (5.3) would contribute). This allows to neglect the sub-leading terms in the solution (5.3). For the longitudinal component we also need the near-horizon limit of $Z(r)$, which can be easily obtained from equation (3.31):

$$
Z^{2} \rightarrow \frac{1}{16 \pi^{2}} \frac{f^{\prime}\left(r_{s}\right)^{2}}{T_{s}^{2}}, \quad r \rightarrow r_{s}
$$

Using the explicit expressions (4.17) in equation (5.1), with $\Psi_{h}=1$, we obtain to the following results:

$$
\begin{aligned}
\kappa_{\perp} & =\frac{1}{\pi \ell_{s}^{2}} b^{2}\left(r_{s}\right) T_{s} \\
\kappa_{\|} & =\frac{16 \pi}{\ell_{s}^{2}} \frac{b^{2}\left(r_{s}\right)}{f^{\prime 2}\left(r_{s}\right)} T_{s}^{3} .
\end{aligned}
$$


We note that $\kappa_{\perp}$ and $\kappa_{\|}$are simply related by $Z^{2} \kappa_{\|}=\kappa_{\perp}$, as it can be read off from equation (5.1), using equation (4.15) and (4.1) and the fact that the small frequency behavior of the wave function is the same for both transverse and longitudinal directions.

In the conformal limit $b(r)=\ell / r, f(r)=1-(\pi T r)^{4}$ and $T_{s}=T / \sqrt{\gamma}, r_{s}=1 /(\pi T \sqrt{\gamma})$, we recover the results of $[18,19]$ for the holographic $\mathcal{N}=4$ SYM:

$$
\begin{aligned}
\kappa_{\perp \mathcal{N}=4} & =\pi \sqrt{\lambda_{\mathcal{N}=4}} \gamma^{1 / 2} T^{3} \\
\kappa_{\| \mathcal{N}=4} & =\pi \sqrt{\lambda_{\mathcal{N}=4}} \gamma^{5 / 2} T^{3} .
\end{aligned}
$$

where $\left(\ell / \ell_{s}\right)^{4}=\lambda_{\mathcal{N}=4}$ is identified with the $\mathcal{N}=4$ 't Hooft coupling, in the $A d S_{5}$ background.

Finite Mass Quarks. For massive quarks, the appropriate boundary condition should be $\Psi_{R}\left(r_{Q}\right)=1$, where $r_{Q}$ is the UV cutoff determined by the value of the quark mass $M_{q}$, using equation (4.3). In this case, equation (5.3) gets modified and reads

$$
\Psi_{R}=\left(\frac{r_{s}-r}{r_{s}-r_{Q}}\right)^{-\frac{i \omega}{4 \pi T_{s}}}[1+\mathcal{O}(\omega)]
$$

Nevertheless, as in the conformal case [22], the results for $\kappa_{\perp}$ and $\kappa_{\|}$remain unchanged, since they are independent of $r_{Q}$. Indeed $r_{Q}$ enters in the wave function, as equation (5.9) shows, and cancels out in the $\omega \rightarrow 0$ limit as we take the product $\Psi_{R}^{*} \Psi_{R}$ in (4.15).

\subsection{The jet-quenching parameter}

As discussed in section 2, the jet-quenching parameters can be defined in terms of the diffusion constants as:

$$
\hat{q}^{\perp}=2 \frac{\kappa^{\perp}}{v}, \quad \hat{q}^{\|}=\frac{\kappa^{\|}}{v} .
$$

The first parameter defines the transverse momentum broadening of heavy quark probes.

There is also a different definition of the jet-quenching parameter, which is related to the perturbative relation between this quantity and an appropriate limit of a Wilson loop joining two light-like lines in Yang-Mills theory (see e.g. [53]). This was the basis of a different holographic calculation of $\hat{q}$, that was carried out in $[11,12]$ in the conformal case, and in [15] for the general backgrounds (3.1), which gives:

$$
\hat{q}_{W L}=\frac{\sqrt{2}}{\pi \ell_{s}^{2}}\left(\int_{0}^{r_{s}} \frac{d r}{b^{2} \sqrt{f(1-f)}}\right)^{-1}
$$

where the subscript $W L$ is introduced to distinguish this definition of the jet-quenching parameter from the original definition (5.10). As in the conformal case, this result differs from the result obtained via the Langevin equation. The reasons for this were analyzed in [59]. 


\subsection{The diffusion constants via the membrane paradigm}

The method of the membrane paradigm that we introduced in section 4.2 allows us to read off the diffusion constants directly from the action (3.33) with no need to derive and solve for the fluctuation equations as in the previous section. The diffusion constants are defined in terms of the symmetric Green's functions by (5.1). Employing the relation (2.14) between the retarded and the symmetric Green's functions and the basic formula of the membrane paradigm, equation (4.22), we arrive at,

$$
\kappa^{a}=2 T_{s} \chi_{R}^{a}=T_{s} Q^{a}\left(r_{s}\right),
$$

where $a=\{\perp, \|\}$. In the second equation above we used the fact that the metric dependence in (4.22) drops out in $2 \mathrm{~d}$.

From the expressions (4.23), and using the near-horizon limit of the function $Z$ from (5.4), we find, unsurprisingly, the same result as equation (5.5), (5.6).

We note that these results establish one of the very few examples of trivial flow as defined in [51], in the sense that the effective couplings $Q$, determined on the horizon membrane, stay unchanged through the flow from the horizon to the boundary. Therefore the dual field theory quantities i.e. the diffusion constants, which should be evaluated on the boundary, can also be computed directly on the horizon due to trivial flow. The other basic example of trivial flow is the shear viscosity $\eta / s$. The reason for trivial flow is that there is no mass term in the fluctuation equations (3.36), because the geometry is flat on the domain-wall directions. For the same reason one expects trivial flow for any transport coefficient that stem from string fluctuations on a generic domain wall background, as long as the fluctuations do not involve the radial direction.

\subsection{A universal inequality: $\kappa_{\|} \geq \kappa_{\perp}$}

From the expressions (5.5), (5.6), one derives the ratio,

$$
\frac{\kappa_{\|}}{\kappa_{\perp}}=\left(\frac{4 \pi T_{s}}{f^{\prime}\left(r_{s}\right)}\right)^{2}=1+4 v^{2} \frac{b^{\prime}\left(r_{s}\right)}{f^{\prime}\left(r_{s}\right) b\left(r_{s}\right)},
$$

where in the last equation, we used the definition of the world-sheet temperature $T_{s}$ in (3.23).

We note that the second term on the r.h.s. of (5.13) is always positive definite in the deconfined phase $T>T_{c}$. This can be seen as follows: First of all, $f^{\prime}(r) b(r)$ is a negative definite quantity at any $r$. This follows from the general relation, see e.g. [42].

$$
f^{\prime}(r)=-\frac{s T}{M_{p}^{3} N_{c}^{2}} b_{E}(r)^{-3}
$$

where $s$ is the entropy density and $b_{E}$ is the Einstein frame scale factor. The left hand side is manifestly negative definite.

Secondly, the quantity $b^{\prime}\left(r_{s}\right)$ is also negative-definite in the deconfined phase. This follows from the fact that, in the type of geometries that confines color, the string frame scale factor $b(r)$ at zero temperature always possesses a minimum at some point $r=r_{*}$. 
Hence $b^{\prime}(r)<0$ for $r<r_{*}$ and $b^{\prime}(r)>0$ for $r>r_{*}$. Moreover, we can argue that, for $T>T_{c}$, the location of the bulk horizon $r_{h}$ should be closer to the boundary than $r_{*}$, i.e. $r_{h}<r_{*}$, otherwise the Wilson loop would have linear behavior, as a result of saturation of the corresponding string at $r_{*}$. Since, the world-sheet horizon is always smaller than the bulk-horizon, it follows that, in the de-confined phase:

$$
r_{s}<r_{h}<r_{*}, \quad \text { for } T>T_{c} \text {. }
$$

Therefore $b^{\prime}\left(r_{s}\right)$ should be negative-definite, and the entire second term on the r.h.s. of (5.13) should be positive-definite. Therefore, we arrive at the universal result that

$$
\kappa_{\|} \geq \kappa_{\perp} \quad \text { for } T>T_{c}
$$

Equality is attained for $v \rightarrow 0$. We check by a numerical computation in section 6 that this inequality is obeyed in the particular background used in that section.

\subsection{A generalized Einstein relation}

We may derive a generalized Einstein relation by relating the diffusion constant (5.5), (5.6) with the friction coefficient $\eta_{D}$ in (2.17).

On the one hand, we have found holographically the relation between the diffusion coefficients $\kappa^{i j}$ and the friction coefficients $\eta^{i j}$ for the Langevin equations in position space (2.24)-(2.25). From equation (4.18) and the definitions (2.13) we arrive at:

$$
\kappa^{i j}=2 T_{s} \eta^{i j}
$$

On the other hand, we can relate $\eta^{i j}$ to the momentum diffusion coefficients $\eta_{D}^{i j}$, by equations (2.26). Therefore we find:

$$
\begin{aligned}
\kappa^{\perp} & =2 T_{s} \gamma M_{q} \eta_{D}^{\perp} \\
\kappa^{\|} & =2 T_{s} \gamma^{3} M_{q}\left(\eta_{D}^{\|}+\left.p \frac{\partial \eta_{D}^{\|}}{\partial p}\right|_{p=\gamma M_{q} v}\right) .
\end{aligned}
$$

These relations lead to an important consistency condition: notice that, by equation (2.23), the coefficient $\eta_{D}^{\|}$must coincide with the zeroth order drag coefficient (3.18), calculated via the classical trailing string solution,

$$
\eta_{D}^{\|}=\eta_{D}=\frac{1}{\gamma M_{q}} \frac{b\left(r_{s}\right)^{2}}{2 \pi \ell_{s}^{2}}
$$

Therefore, consistency requires that, inserting the expression (5.20) for $\eta_{D}^{\|}$in equation (5.19), the resulting expression for $\kappa^{\|}$agree with equation (5.6). This is indeed the case: using the explicit expression (5.20), and the definition $f\left(r_{s}\right)=v^{2}$, we find:

$$
\left(\eta_{D}+\left.p \frac{\partial \eta_{D}}{\partial p}\right|_{p=\gamma M_{q} v}\right)=\frac{1}{\gamma^{2} M_{q}} \frac{b\left(r_{s}\right)^{2}}{2 \pi \ell_{s}^{2}}\left(1+4 v^{2} \frac{b^{\prime}\left(r_{s}\right)}{b\left(r_{s}\right) f^{\prime}\left(r_{s}\right)}\right)
$$


Inserting this expression in the right hand side of equation (5.19), and using the identity (5.13), the resulting expression exactly agrees with $\kappa^{\|}$obtained from the Langevin correlator, (5.6).

Finally, from equation (5.18) and the explicit expression (5.5), we find that $\eta_{\bar{D}} \frac{\text { also }}{}$ equals the drag force coefficient (5.20). This implies that, at the end of the day, the friction term in the momentum diffusion equation is isotropic,

$$
\eta_{D}^{i j}=\frac{1}{\gamma M_{q}} \frac{b^{2}\left(r_{s}\right)}{2 \pi \ell_{s}^{2}} \delta^{i j} \equiv \frac{1}{\tau} \delta^{i j}
$$

The last equality defines the momentum diffusion time $\tau=1 / \eta_{D}$.

We arrive at the generalized Einstein relation:

$$
\tau \kappa_{\perp}=2 M_{q} \gamma T_{s}
$$

where $M_{q}$ is the quark mass and $T_{s}$ is the emergent "world-sheet temperature". (5.23) can be viewed as a generalization of the usual non-relativistic Einstein relation which has the form:

$$
\tau \kappa=2 M_{q} T \text {. }
$$

The modified Einstein relations (5.18) and (5.19) had already been found in [24] in the particular case of the holographic dual of the $\mathcal{N}=2^{*}$ theory, and the results of that analysis fit consistently in the general framework we are considering here.

We note that in the conformal limit, the world-sheet temperature is related to the bulk temperature as

$$
T_{s}=\frac{T}{\sqrt{\gamma}}, \quad \text { conformal }
$$

and (5.23) becomes,

$$
\tau \kappa_{\perp}=2 M_{q} T \sqrt{\gamma}
$$

This is quite different from (5.24) and reduces to it only in the non-relativistic limit $\gamma \rightarrow 1$.

The generalized relation in (5.23) is defined in terms of a set of physical boundary quantities, and the geometric quantity $T_{s}$. In a sense, $T_{s}$ is the temperature that is read by the quark as it moves through the medium. $T_{s}$ provides the answer to the following interesting question: what is the temperature read by a thermometer moving with speed $v$ inside a strongly coupled QGP of temperature $T$.

Note that in the conformal case the answer is universal, and transforms simply with boosts. In the non-conformal case, associated with the Einstein-dilaton system the answer is dynamics dependent.

\subsection{Special limits of the diffusion constants}

In this section we study the diffusion constants (5.5) and (5.6) in the extreme relativistic and non-relativistic limits and express these quantities in terms of thermodynamic functions. 


\subsubsection{Non-relativistic limit}

As $f\left(r_{s}\right)=v^{2}$, in the non-relativistic limit $v \rightarrow 0$, the world-sheet horizon approaches the bulk horizon: $r_{s} \rightarrow r_{h}$. Using the near-horizon expressions for the metric functions, one also finds from (3.23) that $T_{s} \rightarrow T$ in this limit. Finally, we use the expression that relates the entropy density with the scale factor at the horizon, [42],

$$
s=\frac{b_{E}^{3}\left(r_{h}\right)}{4 G_{5}}=4 \pi M_{p}^{3} N_{c}^{2} b_{E}^{3}\left(r_{h}\right)
$$

to obtain

$$
\kappa_{\perp} \rightarrow \frac{2}{\pi}\left(\frac{45 \pi}{4}\right)^{\frac{2}{3}} \frac{\ell^{2}}{\ell_{s}^{2}}\left(\frac{s}{N_{c}^{2}}\right)^{\frac{2}{3}} \lambda_{h}^{\frac{4}{3}} T, \quad v \rightarrow 0,
$$

where $\lambda_{h}$ is the horizon value of $\lambda$. On the other hand, the $\mathcal{N}=4$ result becomes $\kappa_{\perp \mathcal{N}=4} \rightarrow$ $\pi \sqrt{\lambda_{\mathcal{N}=4}} T^{3}$. Hence the ratio becomes,

$$
\frac{\kappa_{\perp}}{\kappa_{\perp \mathcal{N}=4}} \rightarrow \frac{2}{\pi^{2}}\left(\frac{45 \pi}{4}\right)^{\frac{2}{3}} \frac{\ell^{2}}{\ell_{s}^{2}} \frac{1}{\sqrt{\lambda_{\mathcal{N}=4}}}\left(\frac{s}{N_{c}^{2} T^{3}}\right)^{\frac{2}{3}} \lambda_{h}^{\frac{4}{3}}, \quad v \rightarrow 0 .
$$

A similar analysis for the parallel component yields,

$$
\kappa_{\|} \rightarrow \frac{2}{\pi}\left(\frac{45 \pi}{4}\right)^{\frac{2}{3}} \frac{\ell^{2}}{\ell_{s}^{2}}\left(\frac{s}{N_{c}^{2}}\right)^{\frac{2}{3}} T \lambda_{h}^{\frac{4}{3}}, \quad v \rightarrow 0 .
$$

We note that this is exactly the same as (5.28). This is what one expects from the physical perspective. In the non-relativistic limit, the main source of momentum broadening is due to thermal fluctuations in the plasma, that itself is isotropic. Similarly the ratio of QCD and $\mathcal{N}=4$ results also become the same as in (5.29).

\subsubsection{Ultra-relativistic limit}

We consider the opposite limit $v \rightarrow 1$. Here the expression $f\left(r_{s}\right)=v^{2}$ tells us that $r_{s} \rightarrow 0$ hence the world-sheet horizon approaches the boundary. Using the near-boundary expression for $f(r)$ in equation (3.6), and the near-horizon expressions for the metric functions, we find that,

$$
4 \pi T_{s} \rightarrow r_{s}\left(4 \mathcal{C} / \ell^{3}\right)^{1 / 2}\left(1+\mathcal{O}\left(\log ^{-1}\left(r_{s}\right)\right)\right) .
$$

where the constant $\mathcal{C}$ is given in equation (3.7). Upon substitution in (5.5), we finally obtain,

$$
\kappa_{\perp} \rightarrow \frac{\left(45 \pi^{2}\right)^{\frac{3}{4}}}{\sqrt{2} \pi^{2}} \frac{\ell^{2}}{\ell_{s}^{2}} \frac{\left(s T / N_{c}^{2}\right)^{\frac{3}{4}}}{\left(1-v^{2}\right)^{\frac{1}{4}}}\left(-\frac{b_{0}}{4} \log \left(1-v^{2}\right)\right)^{-\frac{4}{3}}, \quad v \rightarrow 1 .
$$

We observe that the result diverges in the extreme relativistic limit $v=1$. However, one obtains a finite expression by considering the ratio with the $\mathcal{N}=4$ result:

$$
\frac{\kappa_{\perp}}{\kappa_{\perp \mathcal{N}=4}} \rightarrow \frac{\left(45 \pi^{2}\right)^{\frac{3}{4}}}{\sqrt{2} \pi^{3}} \frac{\ell^{2}}{\ell_{s}^{2}}\left(\frac{s}{N_{c}^{2} T^{3}}\right)^{\frac{3}{4}} \frac{\left(-\frac{b_{0}}{4} \log \left(1-v^{2}\right)\right)^{-\frac{4}{3}}}{\sqrt{\lambda_{\mathcal{N}=4}}}, \quad v \rightarrow 1 .
$$


Similarly, for the parallel component one finds,

$$
\kappa_{\|} \rightarrow \frac{\left(45 \pi^{2}\right)^{\frac{3}{4}}}{\sqrt{2} \pi^{2}} \frac{\ell^{2}}{\ell_{s}^{2}} \frac{\left(s T / N_{c}^{2}\right)^{\frac{3}{4}}}{\left(1-v^{2}\right)^{\frac{5}{4}}}\left(-\frac{b_{0}}{4} \log \left(1-v^{2}\right)\right)^{-\frac{4}{3}}, \quad v \rightarrow 1 .
$$

Again this is divergent as $v \rightarrow 1$, but the ratio with $\mathcal{N}=4$ result again remains finite in this limit:

$$
\frac{\kappa_{\|}}{\kappa_{\| \mathcal{N}=4}} \rightarrow \frac{\left(45 \pi^{2}\right)^{\frac{3}{4}}}{\sqrt{2} \pi^{3}} \frac{\ell^{2}}{\ell_{s}^{2}}\left(\frac{s}{N_{c}^{2} T^{3}}\right)^{\frac{3}{4}} \frac{\left(-\frac{b_{0}}{4} \log \left(1-v^{2}\right)\right)^{-\frac{4}{3}}}{\sqrt{\lambda_{\mathcal{N}=4}}}, \quad v \rightarrow 1 .
$$

We observe that the parallel and perpendicular components of the diffusion constants asymptote essentially to the conformal result both in the $v \rightarrow 0$ and in the $v \rightarrow 1$ limits. modulo logs in the second case and the appropriate adjustment of relevant parameters like temperature, entropy etc.

We should warn the reader that, as $v \rightarrow 1$, we expect the break down of the treatment based on the local Langevin equation: in fact, for very large $v$ the world-sheet temperature drops to zero, and the auto-correlation time of the Langevin Green's functions, $\tau_{c} \sim T_{s}^{-1}$, diverges. On the other hand, the relaxation time $\tau_{D}=\eta_{D}-1$ stays approximately constant. Therefore, eventually the relation $\tau_{D} \gg \tau_{c}$, necessary for the local treatment, will break down at large enough $v$. In the next subsection we give a detailed discussion of this validity condition.

Another important caveat, when considering the extreme relativistic limit of our results, lies in the fact that they are sensitive to the UV region of the geometry, and as discussed at length in previous work (see e.g. [43]), the details of the gravity theory we are using are not fully reliable in this limit.

\subsection{Time scales and validity of the local approximation}

The results of this section so far were obtained based on two separate approximations concerning the time scales involved. On the one hand, we assume we are in a shorttime approximation, compared to the typical relaxation time. This means that, the quark velocity $v$ can be assumed to be constant only within time scales that are much shorter than the relaxation time $\tau_{D}=1 / \eta_{D}$. On the other hand, the analysis based on the local Langevin equation relies on a long-time approximation, this time compared to the typical time scales entering the Langevin correlators, and determined by the inverse temperature that the quark "feels" as it travels through the plasma. According to our previous discussions this is given by $\tau_{c}=1 / T_{s}$ where $T_{s}$ is the world-sheet temperature (3.23). Therefore our analysis in terms of diffusion constants will be valid only for time scales $t$ such that $\tau_{c} \ll t \ll \tau_{D}$. Existence of time intervals satisfying this condition requires that:

$$
\frac{1}{\eta_{D}} \gg \frac{1}{T_{s}}
$$


Since both $\eta_{D}$ and $T_{s}$ depend non-trivially on the quark momentum, this condition translates in an upper bound on the quark momentum (or velocity), above which the local treatment breaks down. ${ }^{11}$

The relaxation time $1 / \eta_{D}$ is given by (3.18). We can write equation (5.36) more explicitly as

$$
M_{q} \gamma \gg \frac{\ell^{2}}{2 \pi \ell_{s}^{2}} \frac{b^{2}\left(r_{s}\right)}{T_{s}}
$$

We can read this condition as a lower bound on the quark mass. It is most restrictive in the UV (large $v$ ), where it reads,

$$
M_{q} \gg \sqrt{\gamma}\left(\frac{\ell}{\ell_{s}}\right)^{2} \frac{\lambda\left(r_{s}\right)^{\frac{4}{3}}}{2}\left(\frac{\mathcal{C}}{\ell^{3}}\right)^{\frac{1}{4}},
$$

where $\mathcal{C}$ is defined in (3.7) and in the UV region $\lambda\left(r_{s}\right)$ is approximately given by,

$$
\lambda\left(r_{s}\right) \equiv \lambda_{s} \approx-\frac{1}{b_{0} \log \left[\Lambda \ell^{3} /(\mathcal{C} \sqrt{\gamma})\right]} .
$$

We observe that $\lambda\left(r_{s}\right)$ vanishes in the extreme relativistic limit because of the dependence on $\gamma \cdot{ }^{12}$ However, this logarithmic dependence is milder when compared to the explicit dependence on $\gamma$ in (5.38).

Alternatively, equation (5.36) can be read as a condition on the quark velocity, or momentum. For fixed quark mass, and for $v \rightarrow 1, r_{s}$ approaches the AdS boundary, and the right hand side of equation (5.37) scales approximately as $\gamma^{3 / 2}$. Explicitly we find

$$
\sqrt{\gamma} \ll 2 M_{q}\left(\frac{\ell_{s}}{\ell}\right)^{2} \lambda_{s}^{-\frac{4}{3}}\left(\frac{\mathcal{C}}{\ell^{3}}\right)^{-\frac{1}{4}} .
$$

Consequently the condition (5.36) puts an upper bound to the quark velocity.

To obtain an estimate of the upper bound on momentum, in the right hand side of equation (5.37) we can approximate the scale factor as $b(r) \simeq \lambda^{2 / 3}(r)(\ell / r)^{2}$, and replace the quantities $r_{s}$ and $T_{s}$ by by the corresponding conformal expressions, equations (3.24). We arrive at the bound (for an ultra-relativistic quark):

$$
p \ll \frac{1}{4}\left(\frac{\ell_{s}}{\ell}\right)^{4} \frac{M_{q}^{3}}{T^{2}} \lambda_{s}^{-8 / 3} .
$$

For $v$ close to unity, the dependence on $v$ in $\lambda\left(r_{s}\right)$ is very mild and the right hand side can be considered as a constant, depending only by the quark mass, temperature and value of the (holographic) coupling. We will give a numerical estimate of these quantities in the next section, in Improved Holographic QCD.

\footnotetext{
${ }^{11}$ Note that this condition is different than the condition given in [16] for the classical non-relativistic Langevin dynamics that is $1 / \eta \gg 1 / T$. As the thermal behavior of the Green's functions are set by $T_{s}$ rather than $T$, it should be this effective temperature that enters in the validity condition (5.36).

${ }^{12}$ In fact, the numerical studies in the next section shows that the other factor in the log dominates over the $\gamma$ dependence and $\lambda$ can be treated as a constant except in the extreme relativistic limit $v=1$.
} 


\section{Improved Holographic QCD and comparison with data}

In the previous sections we obtained general results for the correlators of world-sheet fluctuations, and for the Langevin diffusion constants, valid in any 5D Einstein-Dilaton theory admitting asymptotically AdS black hole solutions. Here, we will study in detail these results in Improved Holographic QCD, with the potential proposed in [44], whose thermodynamic properties are in good agreement with lattice YM thermodynamics [45] as well as the $T=0$ spectra of glueballs obtained on the lattice.

We take the potential to be, as in $[15,44]$ :

$$
V(\lambda)=\frac{12}{\ell^{2}}\left\{1+V_{0} \lambda+V_{1} \lambda^{4 / 3}\left[\log \left(1+V_{2} \lambda^{4 / 3}+V_{3} \lambda^{2}\right)\right]^{1 / 2}\right\} .
$$

The coefficients $V_{i}$ entering the potential, are fixed as follows (for a detailed discussion see $[15,44])$ :

$$
V_{0}=\frac{8}{9} \beta_{0}, \quad V_{1}=14, \quad V_{2}=\beta_{0}^{4}\left(\frac{23+36 \beta_{1} / \beta_{0}^{2}}{81 V_{1}}\right)^{2}, \quad V_{3}=170 .
$$

where $\beta_{0}$ and $\beta_{1}$ are the first two pure Yang-Mills the beta-function coefficients,

$$
\beta_{0}=\frac{22}{3(4 \pi)^{2}}, \quad \beta_{1}=\frac{51}{121} \beta_{0}^{2} .
$$

The coefficients $V_{0}$ and $V_{2}$ are fixed to match the perturbative YM $\beta$-function, whereas $V_{1}$ and $V_{3}$ are fixed phenomenologically by comparing the equation of state of the model with that of YM on the lattice.

The coefficient $\ell$ is the scale of the asymptotic $A d S_{5}$ space-time at $r=0$ and it sets the energy scale in the field theory. All observables defined holographically using the metric in the Einstein frame are measured in units of $\ell$. For a given class of black hole solutions with fixed UV asymptotics, the value of $\ell$ can be set by matching the mass of the lowest glueball excitation [44].

It may seem that there is an extra scale associated to these models, with respect to $4 \mathrm{D}$ Yang-Mills, where there is a single scale, i.e. the quantity $\Lambda_{\mathrm{QCD}}$ setting the scale of conformal symmetry breaking in the UV. In our model, the analog of the QCD scale emerges as an integration constant that labels different solutions (distinguished by different UV boundary conditions) of the same theory, with $\ell$ fixed. Explicitly, it controls the UV asymptotics of the field $\lambda(r)$, given in equation (3.6), as $\lambda(r)=-\left(b_{0} \log r \Lambda\right)^{-1}+$ $O\left(\log ^{-2} r \Lambda\right)$. Therefore, it may appear we have two independent scales, $\ell$ and $\Lambda$. However, as shown in [39], physical observables depend on $\Lambda$ only via an overall scaling. Therefore, we can choose an arbitrary value for the dimensionless parameter $\ell \Lambda$, and subsequently fix the value of $\ell$ to match some reference energy scale, as explained in the previous paragraph.

The quantities that are computed by probe strings depend on another scale, independent of $\ell$, namely the fundamental string scale $\ell_{s}$. In string-derived models, the ratio $\ell / \ell_{s}$ is known. In phenomenological models on the other hand it must be adjusted to fit observation. For example, the ratio $\ell / \ell_{s}$ can be fixed by comparing the confining string tension 


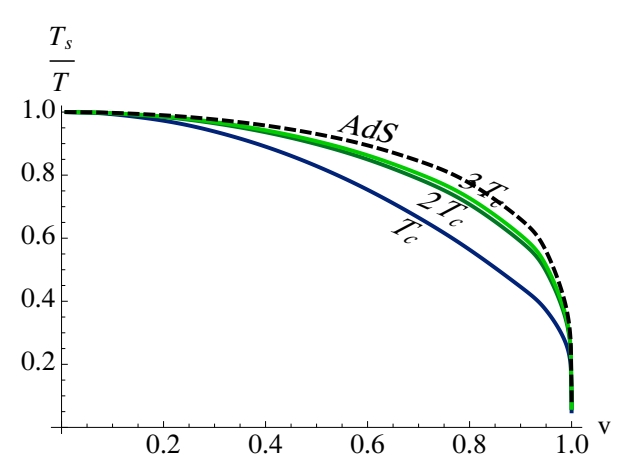

(a)

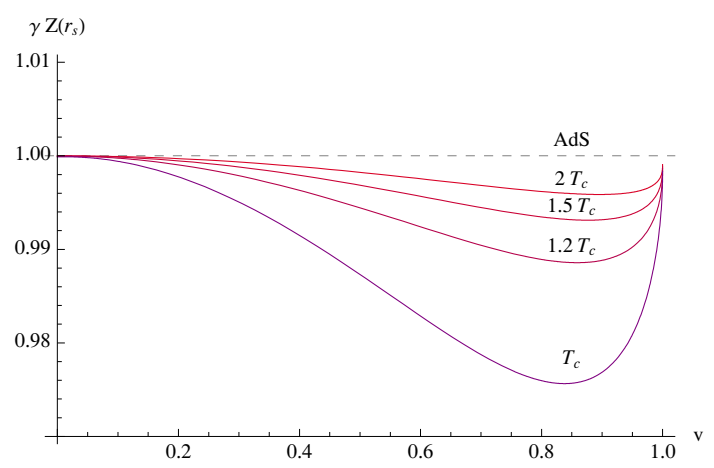

(b)

Figure 1. (a) The ratio of the world-sheet temperature to the bulk black hole temperature, as a function of velocity, for different values of the bulk temperature. The dashed line indicates the AdS-Schwarzschild curve, $T_{s}=T / \sqrt{\gamma}$. (b) The function $\gamma Z\left(r_{s}\right)$ as a function of velocity (with $Z$ defined as in equation (3.31) and $\gamma \equiv 1 / \sqrt{1-v^{2}}$ ), computed numerically varying the velocity, at different temperatures. The dashed line represents the conformal limit, in which $\gamma Z=1$ exactly.

of the holographic model (controlled by the Nambu-Goto action (3.8), hence by $\ell_{s}$ ) to the lattice value $\sigma_{c}=(440 \mathrm{MeV})^{2}$. One finds:

$$
\frac{\ell^{2}}{2 \pi \ell_{s}^{2}}=6.5
$$

This fixes the ubiquitous overall coefficient entering in the diffusion constants, in equations (5.5)-(5.6).

With the potential given by (6.1), we numerically solve Einstein's equation for the metric and dilaton functions $b(r), f(r), \lambda(r)$, to obtain black hole solutions of different temperatures $T$, but obeying fixed UV boundary conditions (for a detailed discussion of the solution procedure, see appendix A of [44]). Once the solutions are given, for each temperature we determine the position of the world-sheet horizon $r_{s}$ as a function of velocity $v$, by numerically solving the equation $f\left(r_{s}\right)=v^{2}$.

From the metric coefficients evaluated at $r_{s}$ we can obtain the world-sheet temperature $T_{s}$, through equation (3.23). The ratio $T_{s} / T$ is plotted as a function of $v$, and for various bulk temperatures, in figure 1 (a). We observe that $T_{s}<T$ for all velocities, and as the bulk temperature increases this ratio approaches the AdS-Schwarzschild curve $\left(T_{s} / T\right)_{\text {AdS }}=\left(1-v^{2}\right)^{1 / 2}$.

Another interesting quantity that provides an indication of how much the backgrounds deviate from the conformal case, is the function $Z(r)$, defined in equation (3.31). For AdSSchwarzschild, this function is exactly constant, $Z(r)=1 / \gamma$. In figure 1 (b) we portray the behavior of $\gamma Z\left(r_{s}\right)$ as a function of velocity, for different bulk temperatures. We observe that as $v \rightarrow 0,1$ this quantity asymptotes to unity, as can also be seen analytically from equation (3.31) by taking the limits $r_{s} \rightarrow 0, r_{s} \rightarrow r_{h}$. Again, as the bulk temperature increases, we move closer to the AdS-Schwarzschild behavior, represented by the dashed line in the graph. 
At this point, we note that figure 1 also provides a confirmation of the universal inequality derived in section 5.4. The function $Z(r)$ of equation (3.31) at the world-sheet horizon is given by $Z\left(r_{s}\right)=f^{\prime}\left(r_{s}\right) / 4 \pi T_{s}$. Then, from equation (5.13) we see that $\kappa_{\|} / \kappa_{\perp}=$ $Z\left(r_{s}\right)^{-2}$. On the other hand, the numerical computation shown in figure 1 implies that,

$$
Z\left(r_{s}\right)<\frac{1}{\gamma} \quad \Rightarrow \quad Z\left(r_{s}\right)^{-2}>\gamma^{2}>1
$$

Therefore the numerics confirm that the inequality $\kappa_{\|} / \kappa_{\perp}>1$ is satisfied.

Knowing the numerical black-hole solutions and the values of $r_{s}$, we can immediately compute the diffusion constants, (5.5)-(5.6). The results are discussed in subsections 6.2, 6.3. On the other hand, in order to compute the full Langevin correlators, we additionally need to solve the world-sheet fluctuation equations. This is analyzed in the next subsection.

\subsection{Correlators and spectral functions}

The retarded correlator of the trailing string fluctuations is given by equation (4.2) as a function of the frequency $\omega$, where the wave-functions $\Psi_{R}^{\perp, \|}(r, \omega)$ are the eigenmodes of the world-sheet fluctuations. From the full retarded propagator, one can further obtain the symmetric one through equation (4.18), and the spectral density through equation (4.19).

To compute the Green's function (4.2) numerically, one must solve the linear fluctuation equations (3.37)-(3.38), with infalling conditions (5.2) at the world-sheet horizon $r_{s}$, and unit normalization at the UV boundary.

The numerical computation makes use of the shooting technique from the world-sheet horizon (specifying the in-falling initial conditions for the wave function and its derivative). Once the full solution is obtained, we normalize it dividing by the value of the solution at the boundary, in order for the wave function to obey the required boundary conditions. The results of the numerical analysis are shown in figures 2 through 4 , and we will discuss them below in more detail.

As discussed in section 4, the real part of the correlator is UV-divergent and therefore it is very sensitive to the cut-off used in the numerical integration. Even if one subtracts the divergent term $\sim \gamma \omega^{2} / \epsilon$, one should be very careful to extract the limit $\epsilon \rightarrow 0$ from the numerics, and eliminate all terms which grow as higher powers of $\omega$, but whose coefficient would vanish at $\epsilon=0$. For example, after subtracting the divergence, the numerical calculation will be dominated by the subleading term in eq. (4.13), which at finite $\epsilon$ grows as $\omega^{4}$, but which is absent when the cut-off is removed.

The imaginary part of the propagator on the other hand does not present these problems, and can be obtained in a clean way from the numerical computation. Since the imaginary part of the retarded correlator is related in a simple way to the symmetric correlator through equation (4.18), we chose to only show plots of the latter, which are discussed below.

In figure 2 and figure 3 the symmetric correlator corresponding to a quark with infinite mass is shown, and it is compared to the WKB result in (4.40), for the transverse and 

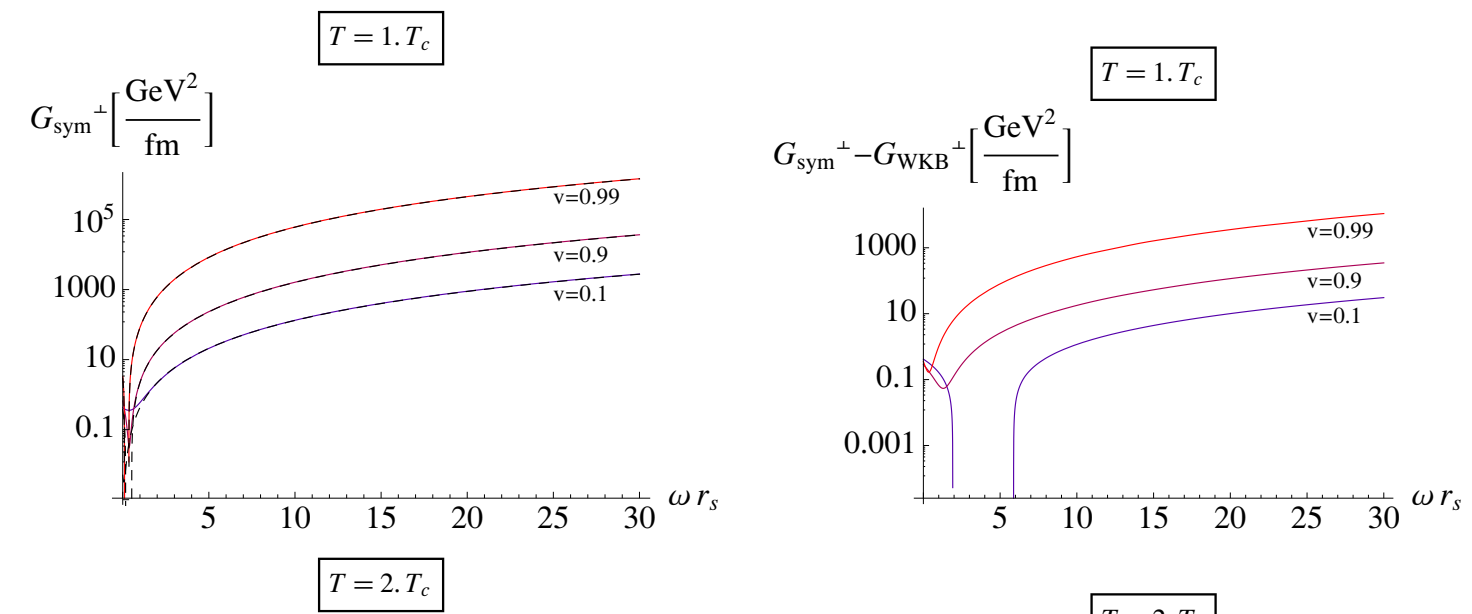

$G_{\text {sym }}{ }^{\perp}\left[\frac{\mathrm{GeV}^{2}}{\mathrm{fm}}\right]$
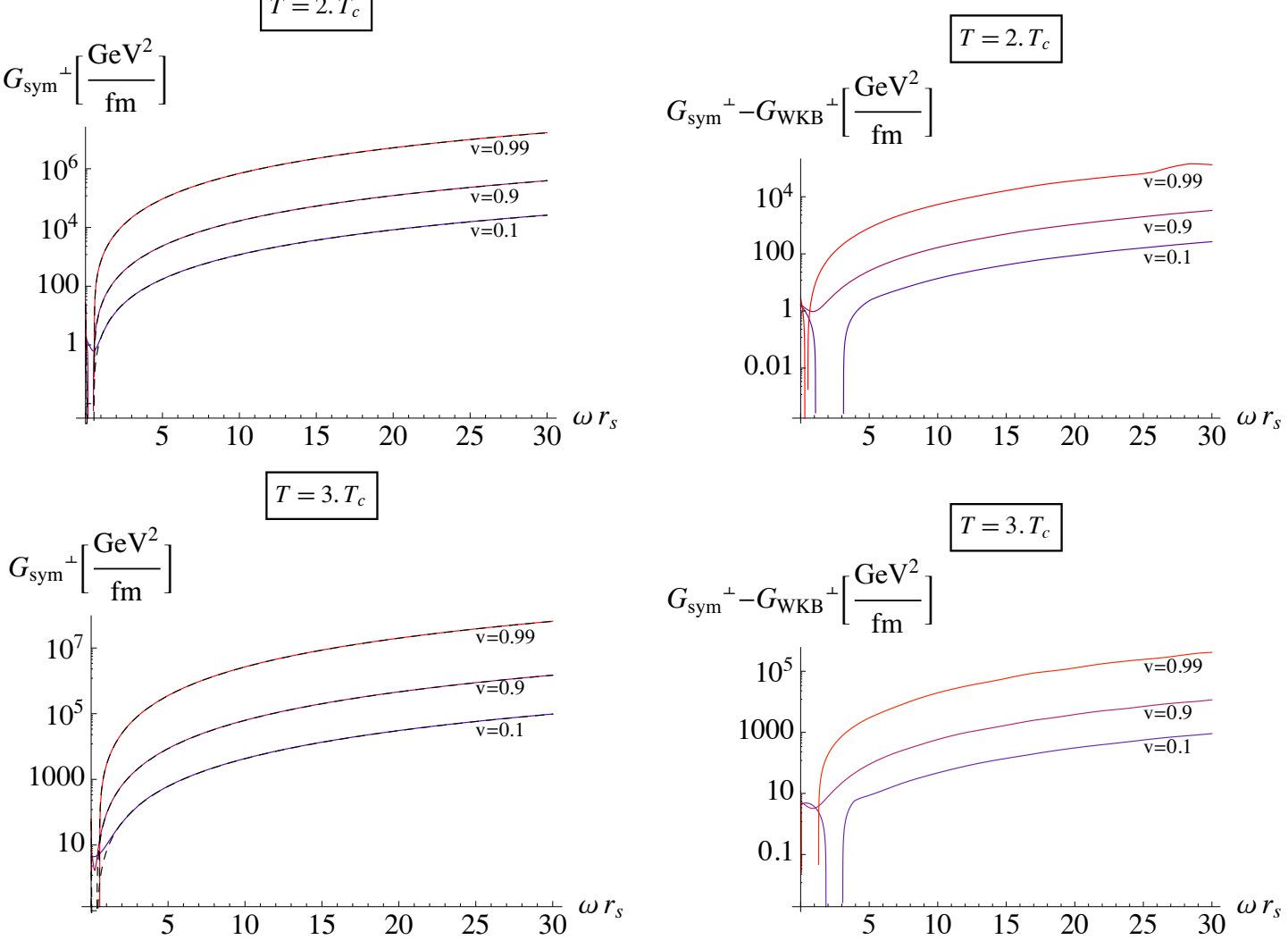

(a)

(b)

Figure 2. (a) The symmetric correlator of the $\perp$ modes by the numerical evaluation (solid line) and by the large-frequency WKB computation of section 4.4 (dashed line), both in the $M_{q} \rightarrow \infty$ limit. (b) Difference of the numerical and WKB results. We show in each plot the curves corresponding to the velocities $v=0.1,0.9,0.99$ and different plots for the temperatures $T=T_{c}, 2 T_{c}, 3 T_{c}$.

longitudinal modes. From these plots we observe that the WKB result is a very good approximation to the spectral densities even at low frequency, which is a priori unexpected. In particular, from comparison with the WKB result, we learn that the symmetric correlators scale with a cubic power-law at large frequency. The difference w.r.t. the WKB 

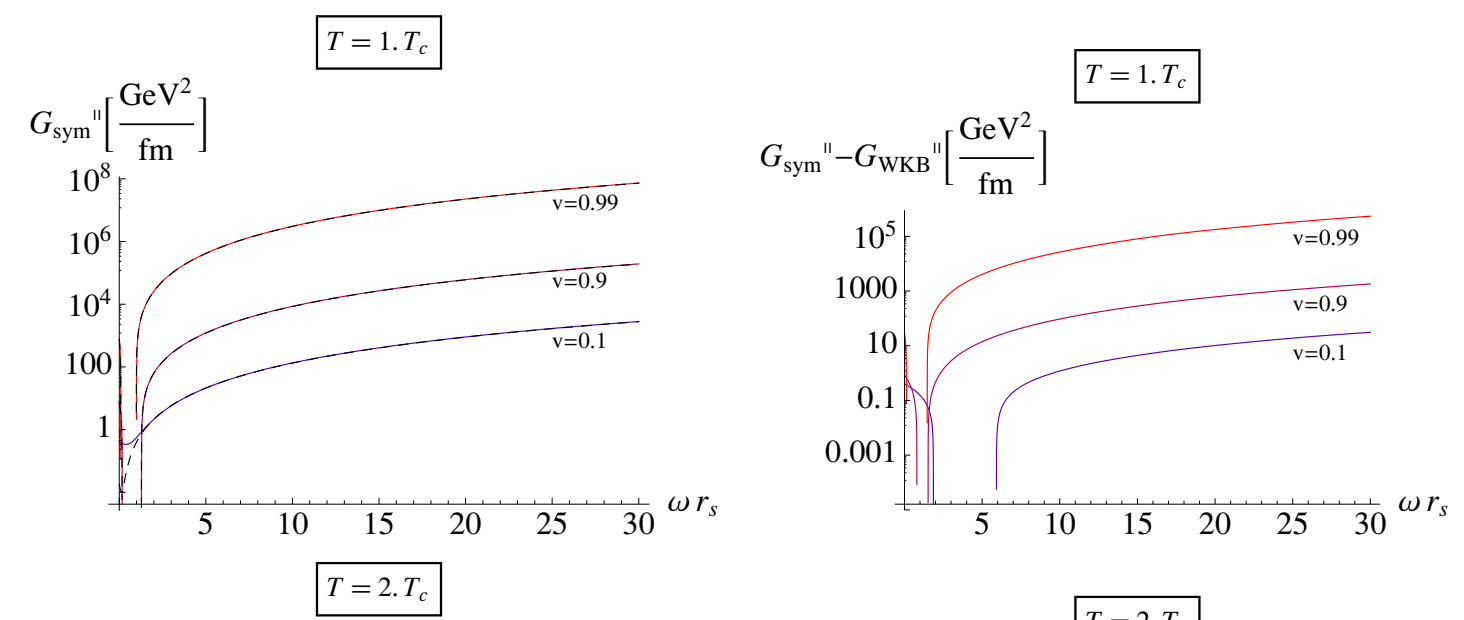

$G_{\text {sym }}$ "[ $\left[\frac{\mathrm{GeV}^{2}}{\mathrm{fm}}\right]$
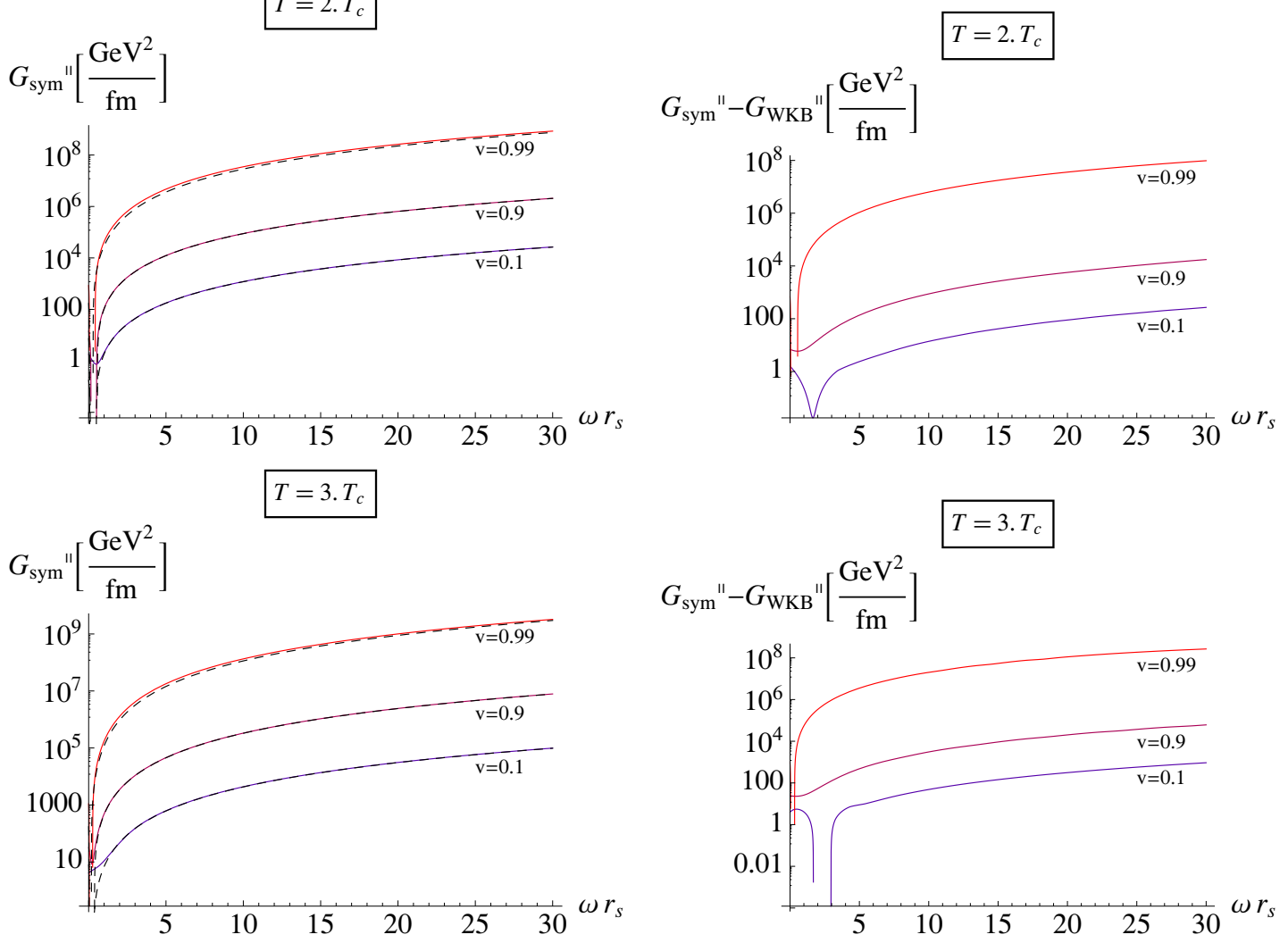

(a)

(b)

Figure 3. (a) The numerical result for the symmetric correlator of the $\|$ modes (solid line) together with the large frequency result from the WKB computation of section 4.4 (dashed line), for $M_{q}=\infty$. (b) Difference between the exact and WKB results. As for the $\perp$ modes, we show in each plot, $T=T_{c}, 2 T_{c}, 3 T_{c}$, the curves corresponding to the velocities $v=0.1,0.9,0.99$.

result is small compared to the value of the correlator (the apparent discontinuity in some of the curves is an artifact due to the logarithmic scale - it is a small bump if plotted on a linear scale).

Figure 4 shows the result for the finite mass correlators and their comparison to the 


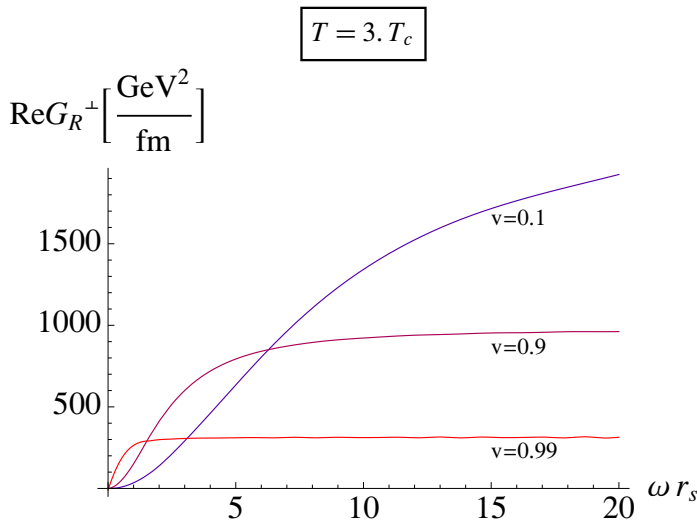

(a)

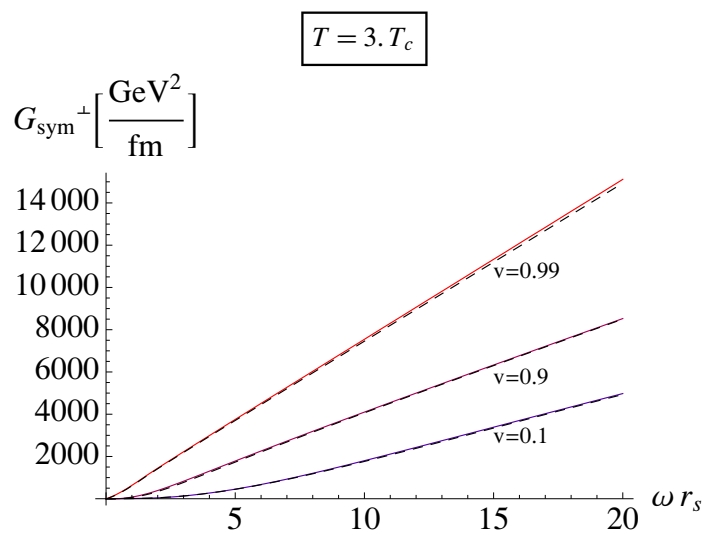

(c)

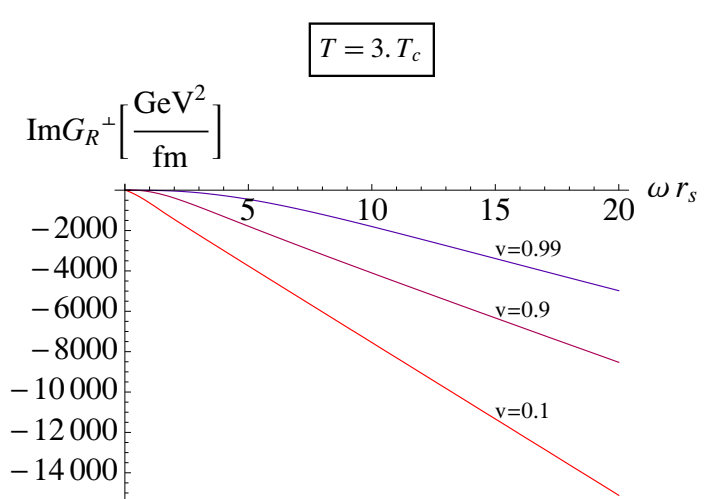

(b)

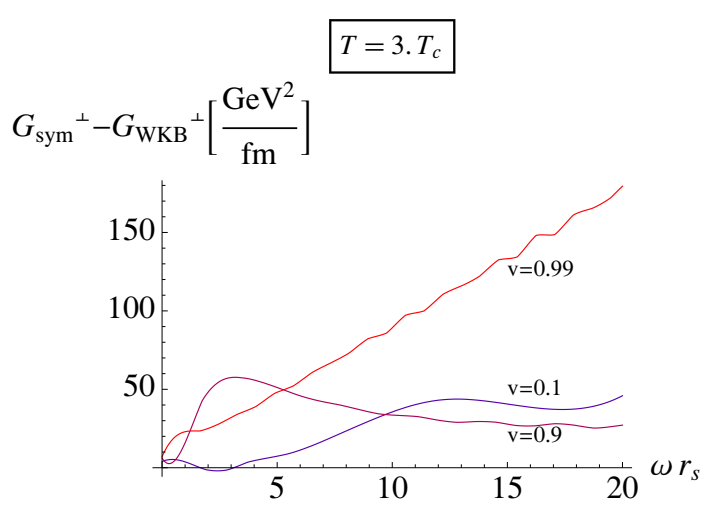

(d)

Figure 4. The retarded correlator ((a) real and (b) imaginary part) for finite but large quark mass, calculated numerically. (c) The symmetric correlator for finite quark mass, from numerical evaluation (solid lines) and WKB result of section 4.4 (dashed lines). (d) The difference between the numerical and WKB result. In each plot we represent the curves corresponding to the velocities $v=0.1,0.9,0.99$. We have taken $T=3 T_{c}$ and $M_{Q}=M_{\text {Charm }}$.

WKB approximation for large frequencies, using the results of section 4.4. The correlators indeed display the linear behavior for large $\omega$ that was derived analytically in section 4.4.

The numeric computation in this case is performed by normalizing the wave function at the cutoff $r_{Q}$. The value of $r_{Q}$ is determined by the quark mass (using equation (4.3)), rather than being given by the regulated boundary $\epsilon$. For the charm and bottom quarks, we take $M_{\text {charm }}=1.5 \mathrm{GeV}$ and $M_{\text {bottom }}=4.5 \mathrm{GeV} .^{13}$

The correlators are also evaluated at $r_{Q}$, following the formula (4.2). We use the results obtained in [15] for the cutoff, yielding $r_{Q} \simeq 1.4$ for the bottom quark and $r_{Q} \simeq 7.5$ for the

\footnotetext{
${ }^{13}$ These values are subject to renormalization in the plasma due to interactions with the media. Therefore, they become temperature dependent. However, as we show in [15], this temperature dependence is very mild in our holographic model, within the relevant temperature range.
} 

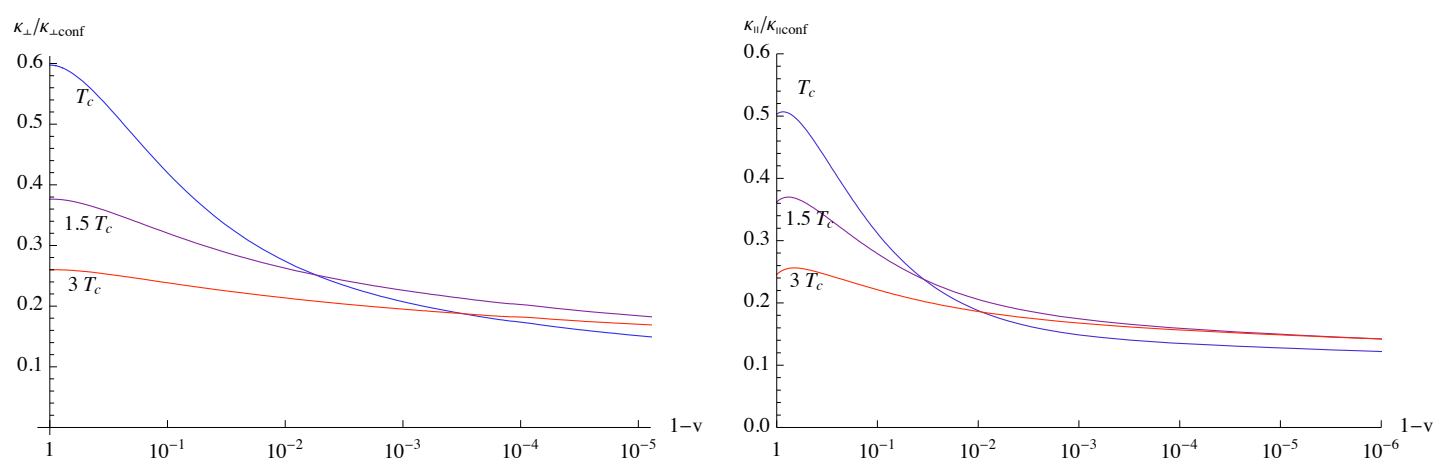

Figure 5. The ratio of the diffusion coefficients $\kappa_{\perp}$ and $\kappa_{\|}$to the corresponding value in the holographic conformal $\mathcal{N}=4$ theory (with $\lambda_{\mathcal{N}=4}=5.5$ ) are plotted as a function of the velocity $v$ (in logarithmic horizontal scale) from equations (5.5)-(5.6). The results are evaluated at different temperatures $T=T_{c}, 1.5 T_{c}, 3 T_{c}$ in the deconfined phase of the non-conformal model.

charm quark. We chose to show in figure 4, as an example, the results for the transverse mode of a charm quark at $T=3 T_{c}$, for different velocities.

\subsection{The jet-quenching parameters}

The diffusion constants $\kappa_{\perp}$ and $\kappa_{\|}$are computed directly, as a function of temperature and velocity, by evaluating equations (5.5)-(5.6) at the world-sheet horizon $r_{s}$ specified by equation (3.15).

To give an idea of the effect of the running of the scalar field, and of the breaking of conformal invariance in our model, in figure 5 we show the ratios of $\kappa_{\perp}$ and $\kappa_{\|}$to the corresponding quantities obtained in the AdS black hole background, equations (5.7)-(5.8) representing strongly coupled $\mathcal{N}=4 \mathrm{SYM}$. The ratios $\kappa / \kappa_{\text {conf }}$ are shown as a function of velocity, at different temperatures. The conformal results are obtained by fixing $\ell_{s}$ by its AdS/CFT relation to the fixed coupling of $\mathcal{N}=4 \mathrm{SYM},\left(\ell / \ell_{s}\right)^{4}=\lambda$. We take as $\lambda=5.5$ as in $[8]$.

From figure 5.1 we observe that, apart from an overall normalization, the nonconformality in this particular model significantly affects the diffusion constants only for temperatures close to $T_{c}$, and for velocities that are not too large. Indeed, if we choose $\lambda \sim 0.5$ instead of $\lambda=5.5$ in the conformal case (just to make the overall magnitudes similar in the comparison), then our result agrees with the conformal result within the $10 \%$ level, in the range $v \gtrsim 0.6$ and for $T \gtrsim 1.5 T_{c}$.

In the rest of this section we will focus on the jet-quenching parameters $\hat{q}$, which are related to the diffusion constants by

$$
\hat{q}^{\perp}=2 \frac{\kappa^{\perp}}{v}, \quad \hat{q}^{\|}=\frac{\kappa^{\|}}{v} .
$$

The Langevin dynamics defines the two independent parameters, $\hat{q}_{\perp}$ and $\hat{q}_{\|}$. The first controls the transverse momentum broadening of a heavy quark probe moving through the plasma, and it is the one usually quoted in relation to experimental results. In this subsection we present the result for the dimensionless quantities $\hat{q} / T_{c}^{3}$, since they do not 

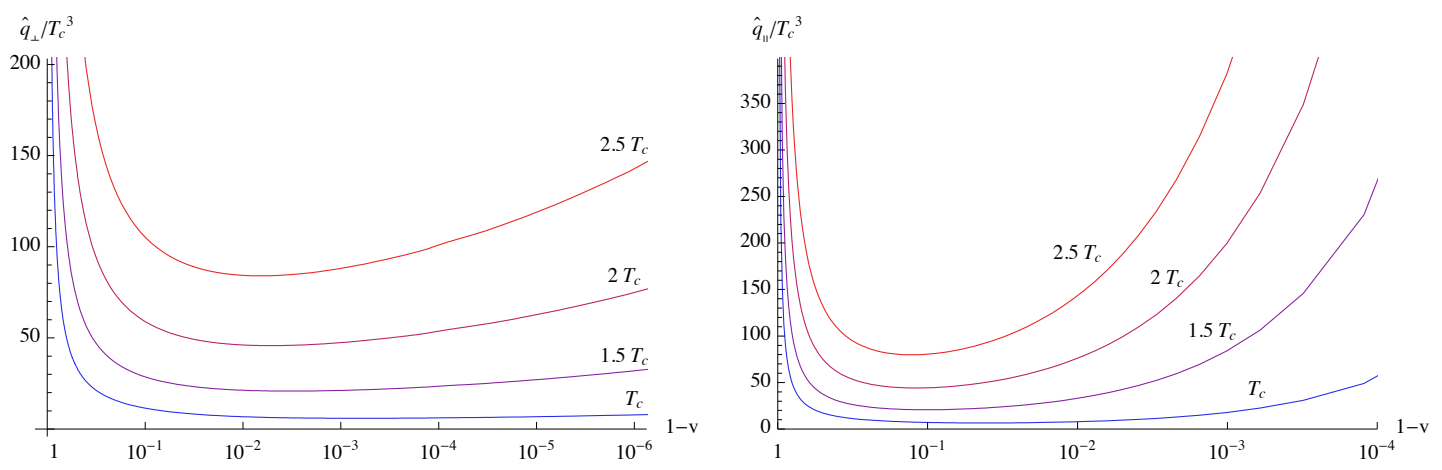

Figure 6. The jet-quenching parameters $\hat{q}_{\perp}$ and $\hat{q}_{\|}$obtained from the diffusion constants (5.5)(5.6), normalized to the critical temperature $T_{c}$, are plotted as a function of the velocity $v$ (in a logarithmic horizontal scale). The results are evaluated at different temperatures.

depend on how we fix the overall energy scale in the holographic QCD model. We will translate the result to physical units in the next subsection.

In figure 6 we plot the two jet-quenching parameters (in units of the critical temperature $T_{c}$ ) as a function of the velocity, for different values of the temperature $T$. The behavior for small $v$ is dominated by the $1 / v$ factor in the definitions (6.5).

We note that the difference between the longitudinal and transverse modes is due to the function $Z$ defined in equation (3.31), which reduces to $f^{\prime}\left(r_{s}\right) / 4 \pi T_{s}$ when evaluated at the world-sheet horizon. More precisely, the relation between the diffusion constants is $\kappa_{\perp}=\kappa_{\|} Z^{2}\left(r_{s}\right)$. In sections 5.6.1 and 5.6.2 we showed that $\gamma Z\left(r_{s}\right) \rightarrow 1$, both when $v \rightarrow 0$ and $v \rightarrow 1$. This is also apparent in the numerical result shown in figure 1 (b).

It is instructive to translate the quark velocity $v$ on the horizontal axis of figure 6 into momentum, $p=M_{q} v \gamma$, where $M_{q}$ is the quark mass. Taking $M_{\text {charm }}=1.5 \mathrm{GeV}$, $M_{\text {bottom }}=4.5 \mathrm{GeV}$, the resulting plots are shown in figure $7 .{ }^{14}$ From these plots, we observe that $\hat{q}_{\perp}$ is almost constant over a wide momentum range, for a relativistic heavy quark. This is not so for $\hat{q}_{\|}$, the difference being due to the extra factor of $Z^{2}\left(r_{s}\right) \sim \gamma^{-2}$ in the latter.

From figure 7 we observe that, for a fixed momentum, $\hat{q}$ increases with temperature, as can also be inferred from the analytic expressions (5.5)-(5.6). This behavior is shown more clearly in figure 8 , that displays $\hat{q}$ as a function of temperature (in units of the critical temperature $T_{c}$ ) for different quark momenta. This is the $\hat{q}_{\perp} \propto T^{3}$ behavior predicted by both the relativistic approximation (5.32) and the non-relativistic one (5.28), once we use the fact that $s \propto T^{3}$ approximately.

It is important to keep in mind that, for a finite quark mass, the trailing string description breaks down when the world-sheet horizon coordinate $r_{h}$ becomes smaller than $r_{Q}$, the point where the trailing string is attached (this is infinite for an infinite quark mass). This translates into an upper bound on the velocity, or momentum, beyond which the setup breaks down, and the string dynamics on the flavor branes becomes important. To estimate this bound, we can use the $A d S_{5}$ relations for the quark mass and world-sheet

\footnotetext{
${ }^{14}$ As shown in [15], thermal corrections to its mass are negligible in our set-up.
} 

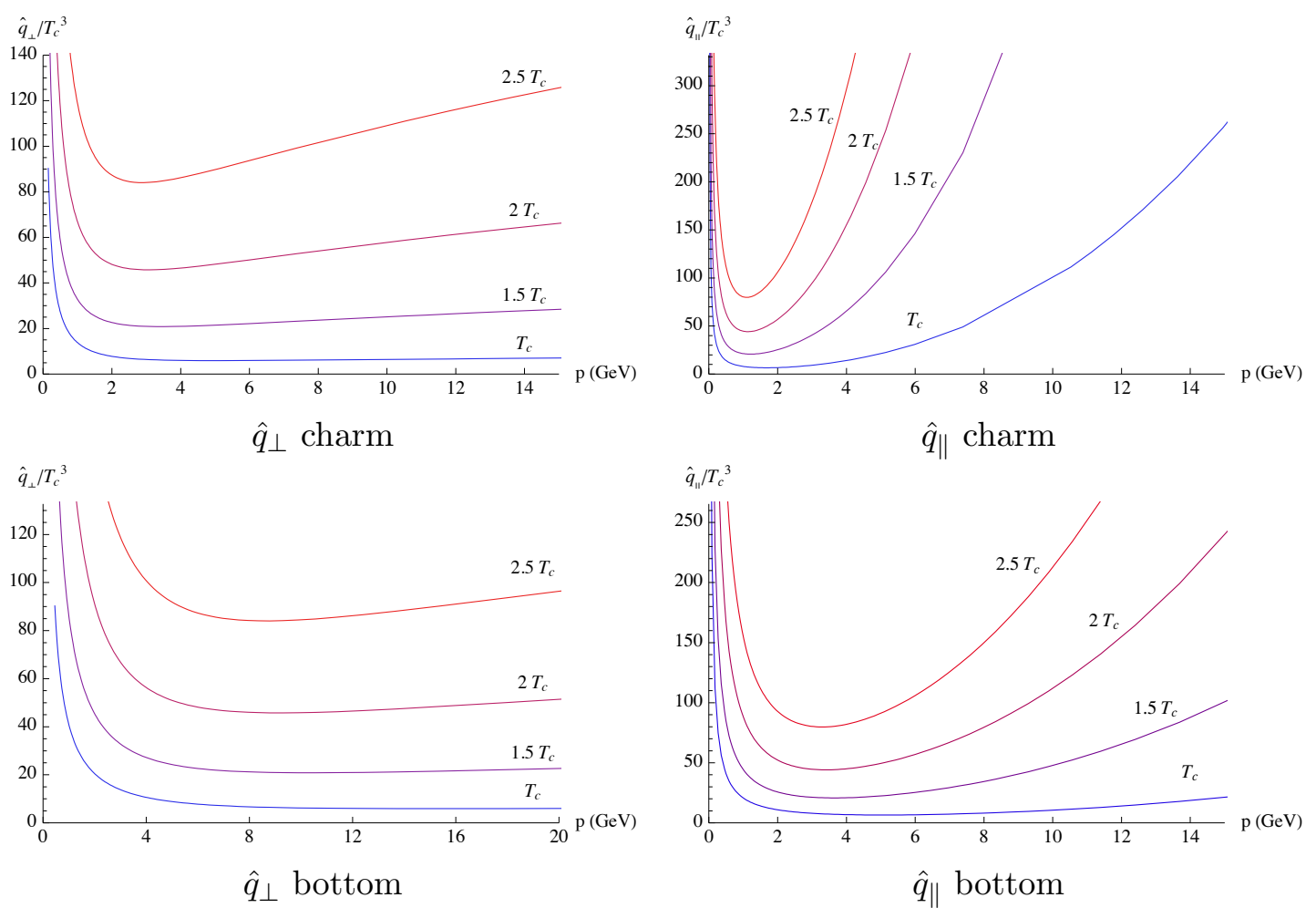

Figure 7. The quantities $\hat{q}_{\perp} / T_{c}^{3}$ and $\hat{q}_{\|} / T_{c}^{3}$ plotted as a function of the quark momentum $p$. The plots for the charm and the bottom quark differ by a scaling of the horizontal direction.

horizon: for a heavy quark $r_{Q}$ is approximately $r_{Q} \simeq\left(\ell^{2} / 2 \pi \ell_{s}^{2}\right) \lambda^{4 / 3}\left(r_{Q}\right) M_{Q}^{-1}$, and for large momentum $p, r_{s} \simeq \gamma^{-1 / 4}(p)(\pi T)^{-1}$, with $\operatorname{gamma}(p)=\sqrt{1+p^{2} / M_{q}^{2}}$. This results in the approximate bound:

$$
p<M_{Q}\left(\frac{M_{Q}}{\pi T}\right)^{2}\left(\frac{2 \pi \ell_{s}^{2}}{\ell^{2}}\right)^{2} \lambda^{-8 / 3}\left(r_{Q}\right)
$$

Notice that the bound is stronger for higher temperatures and lower quark masses.

We estimated numerically the bound on $p$ in the model we are using. The results are displayed in figure 9. From this figure we see that the the bound is easily satisfied for both the Charm and Bottom quarks in the RHIC and LHC regimes, if we use $T_{c} \sim 200 \mathrm{MeV}$

Finally, we need to check what is the allowed range of $p$ such that the local approximation to the Langevin equation is reliable, as discussed in section 5.7. For this to be the case, we need the quantity $T_{s} / \eta_{D}$ to be large. For an ultra-relativistic quark, this condition translates to equation (6.6). In our numerical solution we obtain $\lambda_{s} \sim 3 \times 10^{-2}$. Therefore we expect that, for moderate temperatures the bound is pretty mild. ${ }^{15}$ Taking the above

\footnotetext{
${ }^{15}$ There is a certain degree of arbitrariness in the choice of normalization of $\lambda$. However, changing the normalization of $\lambda$ would result in a value of $\ell / \ell_{s}$ different from the one we are using here, equation (6.4). The important thing is that, ones we insist in fixing the confining string scale at a certain physical value, the quantity $\ell / \ell_{s}$ scales as $\lambda^{-2 / 3}$ under an overall scaling of $\lambda$ [44]. Therefore the bound (6.6) is independent on the overall normalization of $\lambda(r)$.
} 

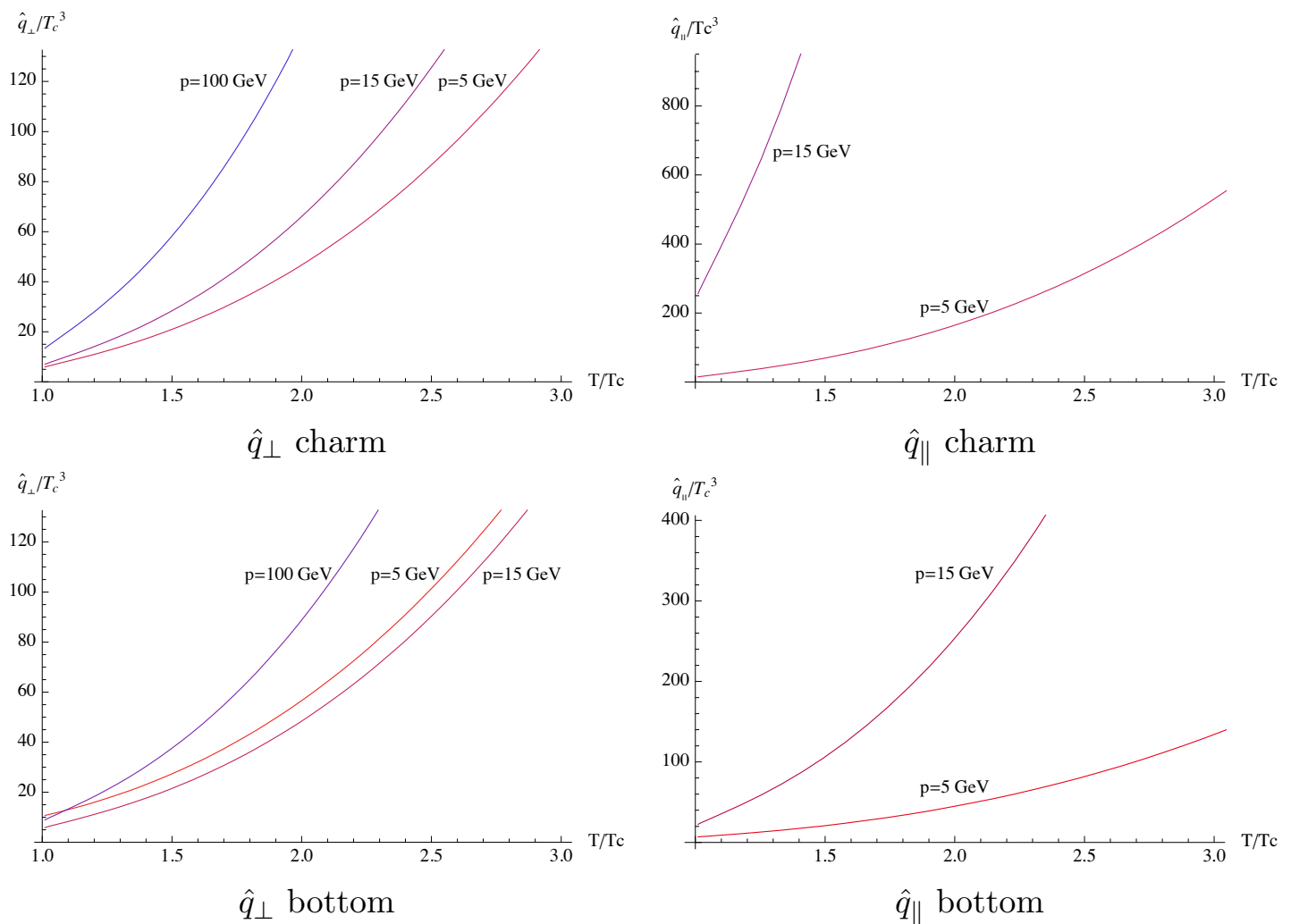

Figure 8. The jet-quenching parameters $\hat{q}_{\perp}$ and $\hat{q}_{\|}$plotted as a function of $T / T_{c}$, for different quark momenta.

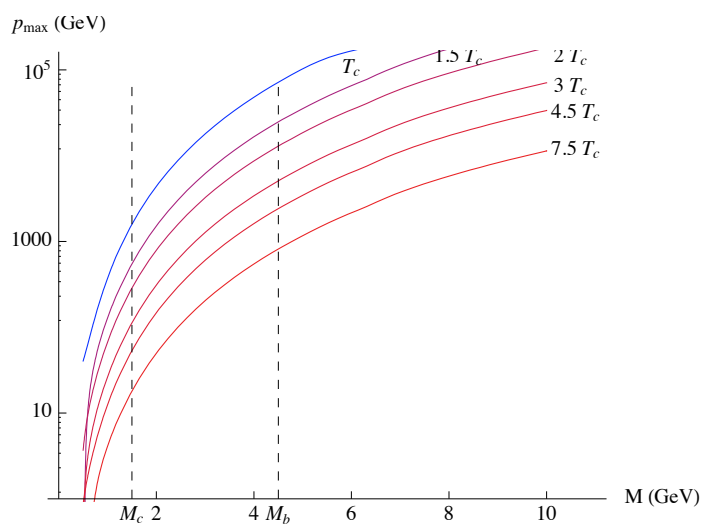

Figure 9. This figure displays the upper bound on momentum $p_{\max }$ (in logarithmic scale) beyond which the trailing string picture ceases to be valid, as a function of the quark mass, and for different temperatures.

result as a reference value for $\lambda_{s}$, and $\ell_{s} / \ell \simeq 0.15$ from equation (6.4), we can rewrite the bound (6.6) more explicitly as follows:

$$
p \ll 1.5 M_{q}\left(\frac{M_{q}}{T}\right)^{2}
$$




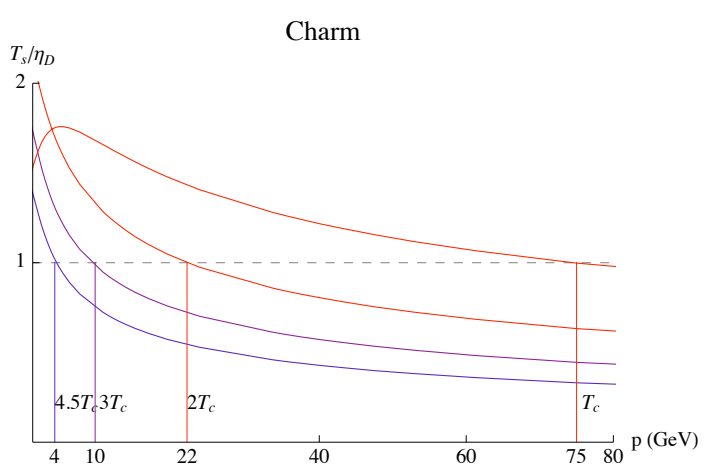

(a)

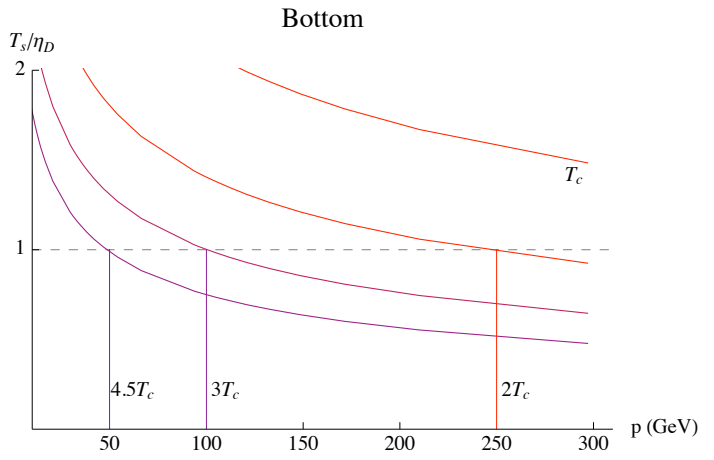

(b)

Figure 10. The quantity $T_{s} / \eta_{D}$ is plotted against quark momentum, for different bulk temperatures. Figures (a) and (b) refer to the charm and bottom quark, respectively. For each temperature, the validity of the local Langevin equation constrains $p$ to the left of the corresponding vertical line, which marks the transition of $T_{s} / \eta_{D}$ across unity.

For example, for the charm quark $\left(M_{q} \simeq 1.5 \mathrm{GeV}\right)$, and close to the critical temperature $T_{c}$, this translates into $p \ll 2 \mathrm{GeV}\left(1.5 \mathrm{GeV} / T_{c}\right)^{2}$.

However, the situation changes dramatically as temperature increases: from eq. (6.7) we observe that the upper limit at temperature $T$ decreases as $\left(T_{c} / T\right)^{2}$. A graphical representation of the validity condition is shown in figure 10, computed numerically from equation (5.41) for both the charm and bottom quarks. The $p$-region in which the diffusion process can be approximated by a local Langevin equation, with constant friction and diffusion coefficients, lies in the left side of the vertical lines (each corresponding to a different temperature). From these plots we observe that the bound is satisfied for momenta up to $\sim 70 \mathrm{GeV}$ (charm) and more than $200 \mathrm{GeV}$ (bottom) at $T=T_{c}$, but for larger temperatures the bounds are much stronger. For example the bounds at $T=3 T_{c}$ are $p_{\text {charm }}<10 \mathrm{GeV}$ and $p_{\text {bottom }}<100 \mathrm{GeV}$.

What these values of $T$ correspond to in terms of actual physical temperature of the QGP, is a subtle question, as we will discuss more in detail in the next subsection. However an order of magnitude estimate can be obtained by setting $T_{c} \approx 180 \mathrm{GeV}$ in these plots. This means that, for RHIC temperatures and momenta, the local approximation remains valid. On the other hand, if the holographic setup is to be applied to ALICE results, it is likely that one should use the full non-local form of the generalized Langevin equation, and the simple parametrization of transverse momentum broadening in terms of $\hat{q}$ breaks down.

\subsection{Comparison with heavy-ion collision observables}

Fit of RHIC data for nuclear modification factors with hydrodynamic simulations prefer a strong jet-quenching parameter for light quarks about $\hat{q}_{\perp}=5-15 \mathrm{GeV}^{2} / \mathrm{fm}$ (for a review of recent results and a more references, see e.g. [53]).

In order to compare our results to QGP observables we need to evaluate the results of the previous section at typical temperature for $\mathrm{QGP} T_{\mathrm{QGP}} \approx 250 \mathrm{MeV}$. 


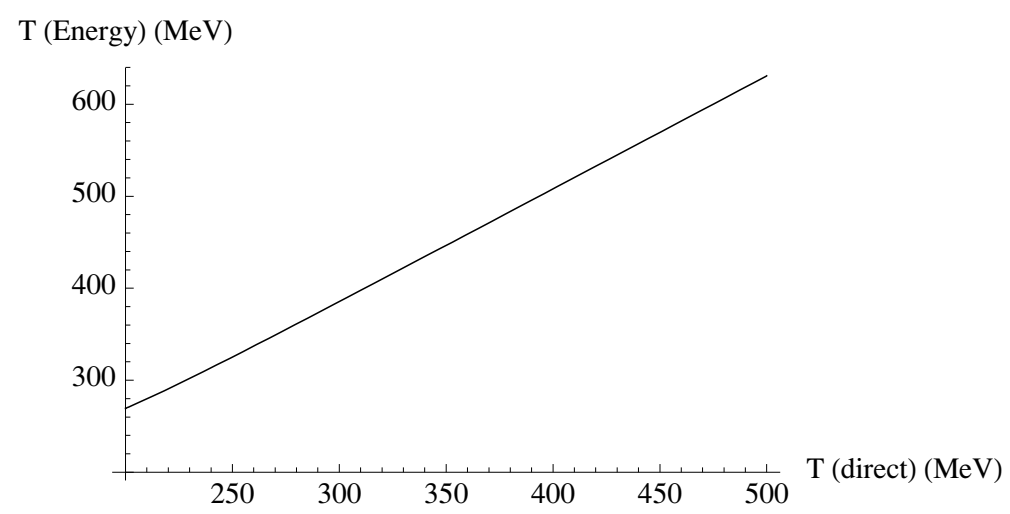

Figure 11. Relation between the temperature in the direct and energy schemes.

However, as discussed in detail in [15] it is not easy to make a direct comparison, because our calculations are made with a pure glue background (neglecting therefore the quark contributions). It was argued recently that quarks contribute importantly in energy loss, beyond their enhancement of the number of degrees of freedom. this was shown to be the case in the thermal $D 3-D 7$ system [60], and the same feature was already noted in [62] in a non-critical model and a in a model based on wrapped $D 5$ branes.

To proceed further we will translate the physical QGP temperature to our $T$. To do this requires picking up a comparison scheme. In a direct scheme one simply takes $T=T_{\mathrm{QGP}}$.

On the other hand, one can argue that the relation between the QGP temperature and that of the holographic model should be such that the energy densities are the same. Energy density scales as the number of degrees of freedom, and the holographic setup we study is supposed to describe pure Yang Mills theory, rather than QCD with three light flavors. Therefore, matching energy densities leads to a holographic temperature $T$ higher than the QGP temperature, due to the different number of degrees of freedom in the two theories. This reasoning leads to the identification of an alternative scheme, referred to as the energy scheme, ${ }^{16}$ where the effective temperature $T$ is related to the real QGP temperature $T_{\mathrm{QGP}}$ by the implicit relation [15]:

$$
\epsilon_{\text {hol }}\left(T_{\text {energy }}\right) \simeq 11.2 T_{\mathrm{QGP}}^{4}
$$

where $\epsilon_{\text {hol }}(T)$ is the energy density of the holographic model.

Computing the $\epsilon_{\text {hol }}(T)$ numerically one obtains the approximate linear relation (as shown in figure 11 ) between the direct scheme and energy scheme temperatures:

$$
\frac{T_{\text {energy }}}{M e V}=23.7+1.2 \frac{T_{\text {dir }}}{M e V}
$$

\footnotetext{
${ }^{16}$ One can also define an entropy scheme, where one matches entropy density rather than energy density. We checked that the numerical results obtained in the energy and the entropy schemes are essentially very close to each other.
} 


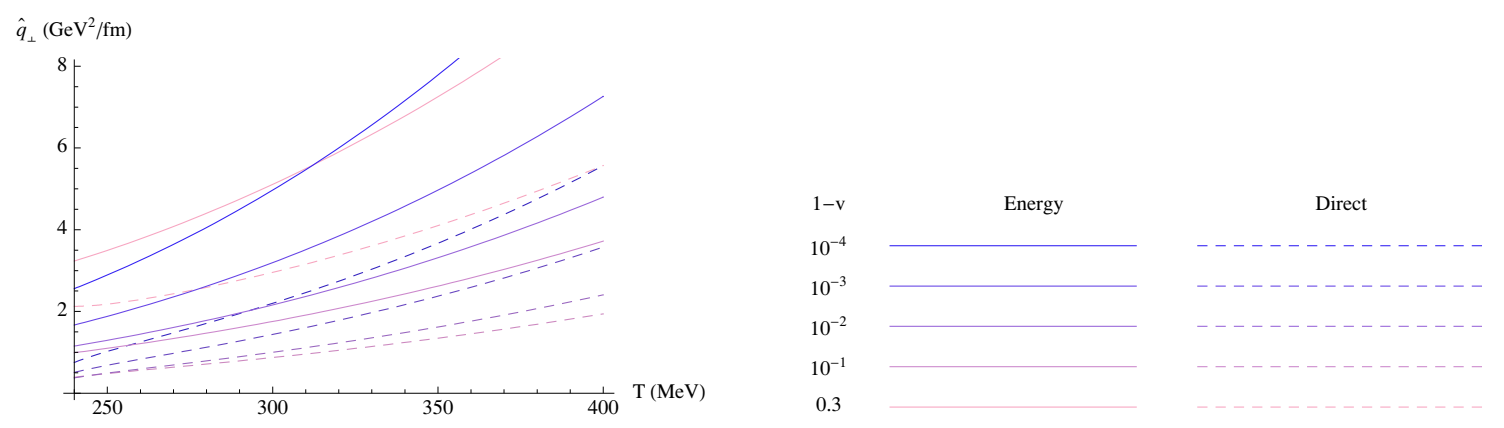

Figure 12. The jet-quenching parameter $\hat{q}_{\perp}$ in the direct (dashed lines) and energy (solid lines) schemes, as a function of temperature, for different quark velocities.
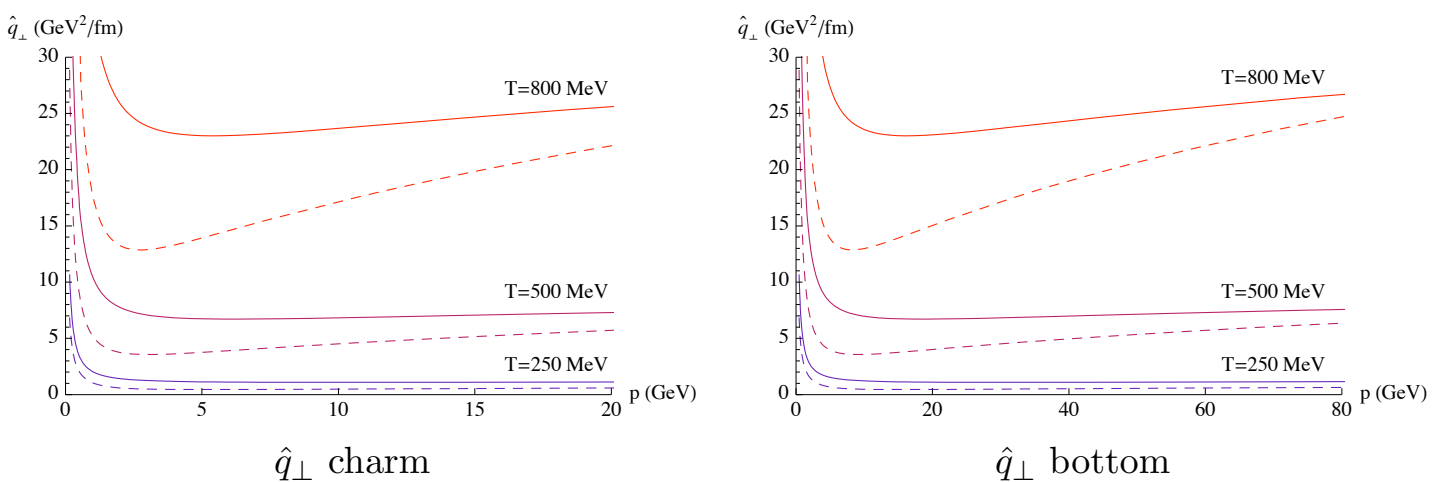

Figure 13. The jet-quenching parameter $\hat{q}_{\perp}$ in the direct (dashed lines) and alternative (solid lines) schemes, as a function of momentum, for different physical values of temperature.

The same $T_{\mathrm{QGP}}$ corresponds to a higher temperature of the holographic model in the energy scheme, than in the direct scheme. Therefore, using the energy scheme to match the QGP temperature results in higher values for $\hat{q}$, than those obtained in the direct scheme. This behavior is apparent in figure 12 .

We are now ready to translate in physical temperatures the results for $\hat{q}_{\perp}$ presented in the previous subsection. This is done in figures 13 and 14, which are analogous to figures 7 and 8 , except that the temperature and $\hat{q_{\perp}}$ are displayed in physical units. In order to express $\hat{q}$ and $T$ in $\mathrm{GeV}^{2} / \mathrm{fm}$ and $\mathrm{MeV}$, respectively, we have to introduce physical energy units. The overall energy scale was fixed, as briefly explained at the beginning of section 6 (and in more detail in [44]), by matching one dimensionfull quantity to its physical value. As in [44], for this purpose we used the lattice value of the lowest glueball mass.

Figure 13 shows $\hat{q}_{\perp}$ in both schemes, as a function of the probe quark momentum, for charm and bottom quarks and for various temperatures in the range relevant for RHIC and for the ALICE experiment at LHC. We observe that, although the values in the energy scheme are higher, at the temperatures relevant for RHIC $(T \approx 250 \mathrm{MeV}), \hat{q}_{\perp}$ varies in the $1-5 \mathrm{GeV}^{2} / \mathrm{fm}$ range except at low momenta where it is substantially higher. A full Langevin fit is necessary in order to ascertain if these numbers fit the data. 

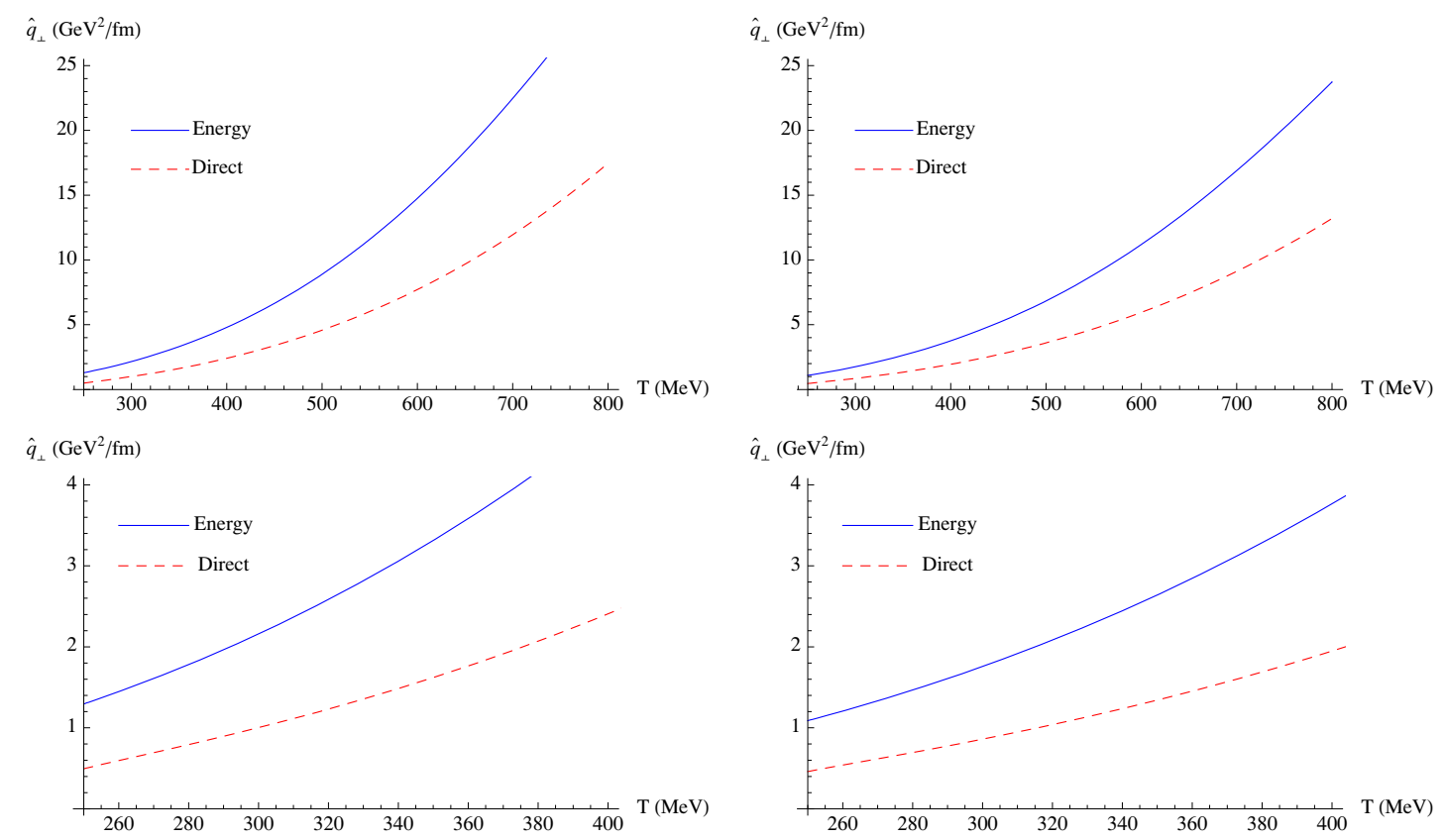

Charm, $p=10 \mathrm{GeV}$

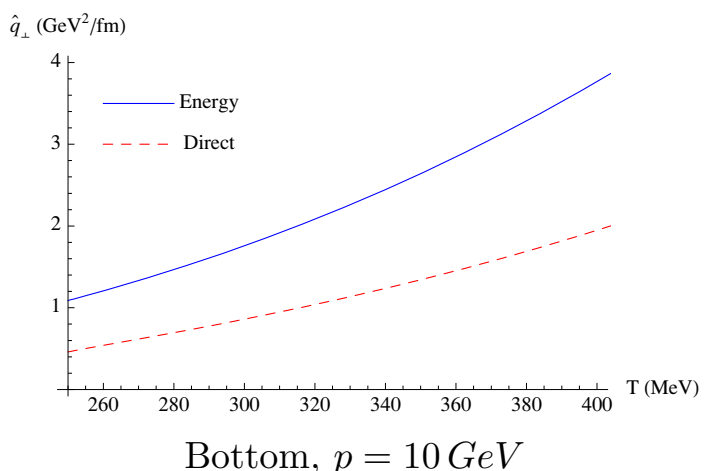

Figure 14. The jet-quenching parameter $\hat{q}_{\perp}$ in the direct (dashed lines) and energy (solid lines) schemes, as a function of temperature, for probe charm and bottom quarks with $p_{T} \approx 10 \mathrm{GeV}$. The bottom plots on show the same functions on a narrower temperature range than the top plots.

For the highest temperature shown in figure 13, $\mathrm{T} \sim 800 \mathrm{MeV}$ (which is not in the range of RHIC, but may be within the reach of ALICE), the predicted value in the energy scheme reaches $\hat{q} \simeq 25 \mathrm{GeV}^{2} / \mathrm{fm}$.

We notice from figure 13 that up to very large momenta $\hat{q}_{\perp}$ is effectively independent of $p$. Therefore one can safely neglect the non-linearity in the Langevin equation for large ranges of $p$. This also allows to pick a reference momentum (say, $p \approx 10 \mathrm{GeV}$ ) and study more closely the behavior of $\hat{q}_{\perp}$ as a function of temperature. This is done in figure 14, which is analogous to figure 8 but with physical units for the temperature.

In figures 13 and 14 one must keep in mind that the parametrization of transverse momentum broadening by $\hat{q}_{\perp}$ fails for momenta that are out of the range validity of the long time approximation to the diffusion process. These bounds are given at the end of the previous section as a function of $T / T_{c}$. In the direct scheme, a plasma temperature of $250 \mathrm{MeV}$ corresponds to $T \simeq T_{c}$ in the holographic model, ${ }^{17}$ but in the energy scheme the same plasma temperature corresponds to $T \simeq 325 \mathrm{MeV}=1.3 T_{c}$ according to the relation (6.8). Similarly, the temperature $T_{\mathrm{QGP}}=800 \mathrm{MeV}$ corresponds to $T \simeq 980 \mathrm{MeV} \simeq 4 T_{c}$ in the energy scheme. Therefore, comparing with figure 10, we deduce that at least for the charm quark, we cannot really trust the analysis in terms of $\hat{q}_{\perp}$ for the highest temperature displayed in figures 13-14, unless we look at momenta smaller than $4 \mathrm{GeV}$. On the other hand, the results shown for the bottom quark are consistent with the long-time approximation.

\footnotetext{
${ }^{17}$ We remind the reader that the critical temperature of the IHQCD model we are using is roughly $T_{c}=247 \mathrm{MeV}[44]$.
} 
Finally, we should remind the reader that our results in the UV are not totally trustable due to the fact that the background used may not describe properly the details of QCD.

\section{Acknowledgments}

We would like to thank J. Casalderrey-Solana, A. Dainese, Y. Foka, E. Iancu, A. Kiessel, M. Panero, G. Policastro, E. Shuryak, U. Wiedemann, for useful conversations. This work was partially supported by a European Union grant FP7-REGPOT-2008-1-CreteHEP Cosmo228644. The work of LM was supported in part by MICINN and FEDER under grant FPA2008-01838 and by the Spanish Consolider-Ingenio 2010 Programme CPAN (CSD200700042).

\section{A Boundary terms and scheme dependence in the propagator}

The form of the retarded propagator we have obtained in section 4, equation (4.2), depends on the form of the action we took as a starting point, namely equation (3.8). As the correlators have UV divergences there is potential scheme dependence in their calculation that we now address. The associated counterterms do not modify the wave equations (3.36), but they change the value of the on-shell action and therefore the correlator.

Since the boundary terms that we add to the action must be real, the scheme dependence can only manifest itself in the definition of $\operatorname{Re} G_{R}(\omega)$. Therefore, it does not affect the physical quantities described in sections 4 and 5, such as the diffusion constants and spectral densities. In the case at hand, as we show below, the only scheme dependence in $\operatorname{Re} G_{R}(\omega)$ amounts to a renormalization in the (heavy) quark mass.

In what follows we study the divergence structure of the action (3.8), expanded to quadratic order in the fluctuations defined in equation (3.26), around the classical trailing string solution. To regulate the action, we cut-off the $r$-integration at $r=\epsilon>0$, and we study the divergences in the $\epsilon \rightarrow 0$ limit.

Starting from equation (3.27) and (3.28), we obtain to quadratic order in the fluctuations,

$$
S_{\mathrm{NG}}=S_{0}+S_{1}+S_{2}+\cdots .
$$

Below, we write explicitly and discuss each term separately.

- Zeroth order.

The zeroth order term reads simply:

$$
S_{0}=-\frac{1}{2 \pi \ell_{s}^{2}} \int d t \int_{\epsilon}^{r_{s}} d r b^{2}(r) Z(r)
$$

For small $r$, the integrand is approximately equal to $(\ell / r)^{2} \lambda^{4 / 3}(r) \gamma^{-1}$. Therefore the integral is dominated by the region around $r \simeq \epsilon$, and it is given approximately by:

$$
S_{0}^{(\mathrm{div})}=\frac{\lambda^{4 / 3}(\epsilon)}{\epsilon} \frac{\ell^{2}}{2 \pi \gamma \ell_{s}^{2}} \int d t,
$$

giving a $1 / \epsilon$ divergence. 
- First order.

The first order term in the fluctuations is a boundary term of the form:

$$
S_{1}=C \int d t \delta X^{\|}(\epsilon, t), \quad C \equiv-\frac{v b^{2}\left(r_{s}\right)}{2 \pi \ell_{s}^{2}} .
$$

Since on-shell $\delta X^{\|}(r=0, t)$ is finite, this term is not divergent.

- Second order.

At quadratic order the action is given by equation (3.34),

$$
S_{2}=-\frac{1}{2 \pi \ell_{s}^{2}} \int d \tau d r \frac{1}{2} H^{\alpha \beta}\left[\frac{1}{Z^{2}} \partial_{\alpha} \delta X^{\|} \partial_{\beta} \delta X^{\|}+\sum_{i=2}^{3} \partial_{\alpha} \delta X^{i} \partial_{\beta} \delta X^{i}\right]
$$

with

$$
H^{\alpha \beta}=\left(\begin{array}{cc}
-\frac{b^{4}}{\sqrt{\left(f-v^{2}\right)\left(b^{4} f-C^{2}\right)}} & 0 \\
0 & \sqrt{\left(f-v^{2}\right)\left(b^{4} f-C^{2}\right)}
\end{array}\right),
$$

It is convenient to write the fluctuation in Fourier space. The solution of the field equations, equations (3.36) reads, close to the boundary:

$$
\delta X^{a}(r, \omega) \simeq C_{s}^{a}(\omega)+C_{v}^{a}(\omega) r^{3} / \lambda^{4 / 3}(r), \quad a=\perp, \|
$$

Inserting the above expression into the action, and using the asymptotic expressions,

$$
H^{t t} \simeq-\gamma b^{2}, \quad H^{r r} \simeq b^{2} / \gamma, \quad Z^{2} \simeq 1 / \gamma^{2},
$$

we observe that the only divergent term as $\epsilon \rightarrow 0$ originates from the terms involving two time derivatives of $\delta X$ :

$$
\begin{aligned}
S_{2}^{(\mathrm{div})} & =\frac{\lambda^{4 / 3}(\epsilon)}{\epsilon} \frac{\ell^{2}}{\gamma} \frac{1}{2} \int d \omega \omega^{2}\left(\gamma^{2}\left|C_{s}^{\perp}(\omega)\right|^{2}+\gamma^{4}\left|C_{s}^{\|}(\omega)\right|^{2}\right) \\
& =\frac{\lambda^{4 / 3}(\epsilon)}{\epsilon} \frac{\ell^{2}}{\gamma} \frac{1}{2} \int d t \gamma^{2}\left(\delta \dot{X}^{\perp}\right)^{2}+\gamma^{4}\left(\delta \dot{X}^{\|}\right)^{2}
\end{aligned}
$$

We can reabsorb both divergences, (A.3) and (A.9), with a single covariant boundary counterterm,

$$
S_{\text {count }}=\Delta M(\epsilon) \int d t \sqrt{\dot{X}^{\mu} \dot{X}_{\mu}},
$$

which corresponds to a renormalization of the quark mass. Indeed, expanding equation (A.10) to second order in $\vec{X}=\vec{v} t+\delta \vec{X}$, we find:

$$
S_{\text {count }} \simeq \frac{\Delta M(\epsilon)}{\gamma}\left\{\int d t+\frac{1}{2} \int d t\left[\gamma^{2}\left(\delta \dot{X}^{\perp}\right)^{2}+\gamma^{4}\left(\delta \dot{X}^{\|}\right)^{2}\right]\right\}
$$

Comparing with equations (A.3) and (A.9) it is clear that the following choice of the leading divergence of $\Delta M$ cancels both the leading and second order divergences:

$$
\Delta M^{(\mathrm{div})}(\epsilon)=-\frac{\lambda^{4 / 3}(\epsilon)}{\epsilon} \frac{\ell^{2}}{2 \pi \ell_{s}^{2}} .
$$


From the discussion above, we conclude that the only boundary term that can lead to a scheme dependence of $\operatorname{Re} G_{R}(\omega)$ can come from the finite part of the counterterm (A.10), with a finite coefficient $\delta m$ completely specified by fixing the renormalized quark mass.

An independent way to see the same effect, is as follows. According to the first line in equation (A.9), a finite counterterm of the form (A.10) with coefficient $\delta m$ would shift $\operatorname{Re} G_{R}(\omega)$ by a term proportional to $\omega^{2}$ :

$$
\Delta \operatorname{Re~G}^{\perp}(\omega)=\frac{\ell^{2}}{2 \pi \ell_{s}^{2}} \delta m \gamma \omega^{2}, \quad \Delta \operatorname{Re} \mathrm{G}^{\|}(\omega)=\frac{\ell^{2}}{2 \pi \ell_{s}^{2}} \delta m \gamma^{3} \omega^{2}
$$

In the Langevin equation, this amounts simply to a finite shift in the quark mass: the generalized Langevin equations (2.2) read, in Fourier space,

$$
\begin{aligned}
& \omega^{2} \gamma M_{q} \delta X^{\perp}(\omega)+G_{R}^{\perp}(\omega) X^{\perp}(\omega)+\xi^{\perp}(\omega)=0, \\
& \omega^{2} \gamma^{3} M_{q} \delta X^{\|}(\omega)+G_{R}^{\|}(\omega) X^{\|}(\omega)+\xi^{\|}(\omega)=0 .
\end{aligned}
$$

after expanding to first order in fluctuations.

The conclusion is that the shifts (A.13) are equivalent to a finite renormalization of the quark mass, $M_{q} \rightarrow M_{q}+\ell^{2} /\left(2 \pi \ell_{s}^{2}\right) \delta m$. Therefore, once the renormalized quark mass is fixed e.g. at zero-temperature by fixing the counterterm, there are no further ambiguities in the two-point function.

\section{B Analytic calculation of the diffusion constants}

Here, we provide a derivation of equations (5.5) and (5.6). These equations follow from the flux (4.15) which can be evaluated at any point, in particular at the horizon. Let us define,

$$
\tilde{\omega}=\frac{\omega}{4 \pi T_{s}},
$$

for notational convenience.

Near the horizon, the solution of the fluctuation equations (3.37) and (3.38) are of the form:

$$
\delta X_{\perp}=C_{\perp}\left(r_{s}-r\right)^{-i \tilde{\omega}}, \quad \delta X_{\|}=C_{\|}\left(r_{s}-r\right)^{-i \tilde{\omega}} .
$$

In (4.15) we also need the near-horizon expression for the r-r component of the world-sheet metric $H^{r r}$ equation (3.35). Using the definitions $C=v b\left(r_{s}\right)$ and $f\left(r_{s}\right)=v^{2}(3.20)$ and the definition of $T_{s}$ in (3.23), we find:

$$
H^{r r} \rightarrow 4 \pi T_{s} b^{2}\left(r_{s}\right)\left(r-r_{s}\right) .
$$

Substituting (B.2) and (B.3) in (4.15) yields the flux near the horizon (and everywhere):

$$
\operatorname{Im} G_{R}=\left\{\begin{array}{l}
\left|C_{\perp}\right|^{2} b^{2}\left(r_{s}\right) \omega, \\
\left|C_{\|}\right|^{2} \frac{b^{2}\left(r_{s}\right)}{Z^{2}\left(r_{s}\right)} \omega,
\end{array}\right.
$$

From (3.31) we also find,

$$
Z(r) \rightarrow \frac{f^{\prime}\left(r_{s}\right)}{4 \pi T_{s}}, \quad r \rightarrow r_{s}
$$


Therefore the calculation is reduced to finding the coefficients $C_{\perp}$ and $C_{\|}$. This can be done by matching the near-horizon solution (B.2) for small $\omega$ to the exact analytic solution of the fluctuation equations again for small $\omega$. We give details for $\delta X_{\perp}$, the other component is entirely analogous. For small $\omega$ (B.2) expands as,

$$
\delta X_{\perp} \approx C_{\perp}-i \tilde{\omega} C_{\perp} \log \left(r_{s}-r\right) .
$$

In the strict $\omega=0$ limit this gives

$$
\delta X_{\perp}=C_{\perp}, \quad \omega=0 .
$$

On the other hand, (3.37) can be solved exactly in the strict $\omega=0$ limit:

$$
\delta X_{\perp}=C_{1}+C_{2} \int_{0}^{r} \frac{d t}{\sqrt{\left(f-v^{2}\right)\left(b^{4} f-C^{2}\right)}} .
$$

Requiring unit norm on the boundary fixes $C_{1}=1$. The second term diverges at the horizon, therefore in the strict $\omega=0$ limit, one should have $C_{2}=0$. Therefore one has,

$$
\delta X_{\perp}=1,
$$

in the strict $\omega=0$ limit. Since the solution (B.9) is valid everywhere, including the horizon, one can match it with (B.7) and obtain

$$
C_{\perp}=1 .
$$

More generally, the solution at all $r$ can be written as

$$
\delta X_{\perp}=C_{\perp}(\omega)\left(r_{s}-r\right)^{-i \tilde{\omega}}\left[1+D_{1 \perp}(\omega)\left(r_{s}-r\right)+D_{2 \perp}(\omega)\left(r_{s}-r\right)^{2}+\mathcal{O}\left(\left(r_{s}-r\right)^{3}\right)\right] .
$$

Expanding $C_{\perp}$ and $D_{\perp}$ around $\omega=0$ (the regular expansion is guaranteed by the regularity of the $\omega=0$ solution), we get

$$
\begin{aligned}
\delta X_{\perp}= & C_{\perp}(0)\left(r_{s}-r\right)^{-i \tilde{\omega}}\left[1+\left(D_{1 \perp}(0)\left(r_{s}-r\right)+\mathcal{O}\left(\left(r_{s}-r\right)^{2}\right)\right)+\right. \\
& \left.\left(C_{\perp}^{\prime}(0) / C_{\perp}(0)+D_{1 \perp}^{\prime}(0)\left(r_{s}-r\right)+\mathcal{O}\left(\left(r_{s}-r\right)^{2}\right)\right) \omega+\mathcal{O}\left(\omega^{2}\right)\right] .
\end{aligned}
$$

Now, equation (B.9) implies that $D_{1 \perp}(0)=0$ - and so on for all the $D_{i \perp}(0)$ - and $C_{\perp} \equiv C_{\perp}(0)=1$. Hence the solution for all values of the radial coordinate is (5.3):

$$
\delta X_{\perp}=\left(r_{s}-r\right)^{-i \tilde{\omega}}\left[1+\tilde{C}_{1 \perp}(r) \omega+\mathcal{O}\left(\omega^{2}\right)\right]
$$

with $\tilde{C}_{1 \perp}(r) \simeq C_{\perp}^{\prime}(0) / C_{\perp}(0)+D_{1 \perp}^{\prime}(0)\left(r_{s}-r\right)$, close to the horizon.

In passing, we note that slightly away from the $\omega=0$ limit one can allow for the second term in (B.8), and expanding the integrand near the horizon, one obtains,

$$
\delta X_{\perp}=C_{1}+\frac{C_{2}}{b^{2}\left(r_{s}\right) 4 \pi T_{s}} \log \left(r_{s}-r\right) .
$$


Matching this with (B.6) one can also determine $C_{2}$ in the small $\omega$ limit: $C_{2}=-i \omega b^{2}\left(r_{s}\right)$. This information is not required to calculate the diffusion coefficients.

Use of (B.10) in (B.4) and eventually in (5.1) yields the desired result (5.5) (after including the string tension in front of the world-sheet action). The discussion for the parallel component is similar. Solving the fluctuation equation (3.38) for $\omega=0$ and by matching (B.2) one finds $C_{\|}=1$ and using this and (B.5) in (B.4) yields (5.6).

\section{Details of the WKB approximation}

We follow the steps outlined in section 4.4. It is convenient to define the dimensionless variables $x \equiv r / r_{s} \in(0,1)$ and $\omega_{s} \equiv \omega r_{s}$. The analog Schrödinger equation is

$$
-\psi^{\prime \prime}+V_{s}(x) \psi=0, \quad V_{s}(x)=-\frac{\omega_{s}^{2} b^{4}}{R^{2}}+\frac{1}{2}(\log \mathcal{R})^{\prime \prime}+\frac{1}{4}(\log \mathcal{R})^{\prime 2} .
$$

where

$$
\mathcal{R}=\left\{\begin{array}{l}
R \\
R / Z^{2}
\end{array}, \quad \mathcal{Z}= \begin{cases}1 & \perp \\
Z & \|\end{cases}\right.
$$

and the functions $R(x)$ and $Z(x)$ are:

$$
R=\sqrt{\left(f-v^{2}\right)\left(b^{4} f-C^{2}\right)}, \quad Z=b^{2} \sqrt{\left(f-v^{2}\right) /\left(b^{4} f-C^{2}\right)}
$$

We divide the range $0<x<1$ in three regions, in each of which we use different approximations to solve the Schrödinger equation. The following discussion holds for both $\perp$ and || fluctuations, so we will not make any distinction from now on.

1. Near Boundary: $x \ll 1$

In this region we have the following asymptotics:

$$
R(x) \sim b^{2}(x) / \gamma, \quad Z \sim 1 / \gamma, \quad x \ll 1,
$$

and the Schrödinger potential is approximately

$$
V_{s} \simeq-\gamma^{2} \omega_{s}^{2}+(\log b)^{\prime \prime}+(\log b)^{\prime 2}, \quad x \rightarrow 0 .
$$

In the near-boundary region the Einstein frame scale factor becomes that of AdS space-time, and we have:

$$
b(x) \simeq \frac{\ell}{r_{s}} \frac{\lambda^{2 / 3}(x)}{x}
$$

One important property of this region, is that the quantity $r \lambda^{\prime} / \lambda$ is small. The reason is that the field equation for $\lambda(r)$ is $[38,39]$ :

$$
\lambda^{\prime}(r) \sim \frac{b_{E}(r)}{\ell} \lambda^{2} \sim \frac{\lambda^{2}}{r} \Rightarrow r \frac{\lambda^{\prime}}{\lambda} \sim \lambda \ll 1
$$

where $b_{E}(r) \sim \ell / r$ is the Einstein frame scale factor close to the boundary.

As a consequence, all terms proportional to $r \lambda^{\prime} / \lambda$, or corresponding higher derivative terms in $\lambda$, can be treated, to a first approximation, as subleading in an expansion in $\lambda$. 
2. Near Horizon: $x \simeq 1$

In this region we have:

$$
R(x) \simeq\left(4 \pi T_{s} r_{s}\right) b^{2}\left(r_{s}\right)(1-x), \quad Z \simeq \frac{f^{\prime}\left(r_{s}\right)}{4 \pi T_{s}}
$$

leading to:

$$
V_{s}(x) \simeq-\left(\tilde{\omega}^{2}+\frac{1}{4}\right) \frac{1}{(1-x)^{2}}, \quad x \rightarrow 1
$$

where $\tilde{\omega} \equiv \omega / 4 \pi T_{s}$.

3. WKB Region: $x_{t p} \ll x<1$

This is the classically allowed region, where $V_{s}(x)<0$. For large $\omega_{s}$, the first term in equation (C.1) dominates, except close to the turning point $x_{t p}$, where the contributions of the other terms get large,

$$
V_{s} \simeq-\frac{\omega_{s}^{2} b^{4}}{R}, \quad x_{t p} \ll x<1
$$

Since, for any large but finite $\omega_{s}, V_{s}(x=0)=+\infty$, the turning point for large $\omega_{s}$ is close to the boundary and it is found by solving the equation $V_{s}(x)=0$ in this limit, i.e. using $V$ in the form (C.5). Keeping in mind that derivatives of $\lambda(x)$ close to the boundary produce corrections of $O(\lambda) \ll 1$, we find the turning point for large $\omega_{S}$ :

$$
x_{t p}=\frac{\sqrt{2}}{\omega_{s} \gamma}(1+O(\lambda)), \quad \omega_{s} \gg 1 .
$$

The crucial fact is that, for large $\omega_{s}, x_{t p} \ll 1$, and regions 1 and 3 overlap. On the other hand, regions 2 and 3 overlap close to $x \sim 1$. Therefore, the solution in the WKB region can be used to connect the near-boundary and near-horizon asymptotics.

To find the wave-function in the large $\omega_{s}$ regime, we follow the steps outlined in section 4.4.

1. Consider first the WKB region. The two independent solutions to $-\ddot{\psi}+V \psi=0$ in the region $V \ll 0$ are written, in the WKB approximation:

$$
\psi_{1} \sim \frac{1}{\sqrt{p}} \cos \int^{x} p, \quad \psi_{2} \sim \frac{1}{\sqrt{p}} \sin \int^{x} p, \quad p(x) \equiv \sqrt{-V_{s}(x)}=\frac{\omega_{s} b^{2}}{R} .
$$

Explicitly, the general solution has the form:

$$
\psi_{w k b}=C_{1} \frac{\sqrt{R}}{b} \cos \left[\int_{0}^{x} \frac{\omega_{s} b^{2}}{R}\right]+C_{2} \frac{\sqrt{R}}{b} \sin \left[\int_{0}^{x} \frac{\omega_{s} b^{2}}{R}\right] \quad x_{t p} \ll x \leq 1
$$

In the equation above, we made the arbitrary choice $x=0$ for the lower integration limit, in order to avoid ambiguities in the definitions of the integration constants. 
2. Consider now the near-horizon region. There, we can solve Schrödinger's equation with the potential (C.9). The solution with in-falling boundary condition at the horizon is

$$
\psi_{h} \simeq C_{h}(1-x)^{-i \tilde{\omega}+\frac{1}{2}}, \quad \tilde{\omega} \equiv \frac{\omega}{4 \pi T_{s}} \quad x \simeq 1,
$$

Since both forms (C.13) and (C.14) are valid in the near-horizon region and for large $\omega$, we can relate the coefficients by evaluating (C.13) near the horizon. In order to use the near-horizon expansion of the equation (C.8) in the integrands appearing in equation (C.13), we change the extremum $x_{0}$ to another point $x_{1}$ belonging to the horizon region. This introduces a common phase shift, $\theta=\int_{0}^{x_{1}} \omega_{s} b^{2} / R$, in the sine and cosine functions. Taking this into account, the near-horizon expansion of equation (C.13) reads:

$$
\begin{aligned}
\psi_{w k b} \simeq & \left(4 \pi T_{s} r_{s}\right)^{1 / 2}(1-x)^{1 / 2}\left\{C_{1} \cos [\theta-\tilde{\omega} \log (1-x)]+\right. \\
& \left.+C_{2} \sin [\theta-\tilde{\omega} \log (1-x)]\right\}, \quad x \rightarrow 1 .
\end{aligned}
$$

Comparing equations (C.15) and (C.14) gives the relations:

$$
-i C_{2}=C_{1}=\frac{C_{h}}{\left(4 \pi T_{s} r_{s}\right)^{1 / 2}} e^{-i \theta}
$$

3. Next, we consider the boundary region, $x \ll 1$. Here the potential has the form (C.5). Since the potential diverges as $1 / x^{2}$, a WKB treatment is impossible all the way to $x=0$, so we must resort to another method. The strategy we follow is that of an expansion in the derivatives of $\lambda(x)$, more precisely in the small quantity $r \lambda^{\prime} / \lambda \sim$ $O(\lambda) \ll 1$. This will allow us to write an approximate expression for the solution, valid for any $\omega_{s}$.

Using the approximation for the metric in (C.5), the second entering the nearboundary potential can be written as:

$$
(\log b)^{\prime \prime} \simeq \frac{1}{x^{2}}\left(1+\frac{2}{3} \frac{x^{2} \lambda^{\prime \prime}}{\lambda}-\frac{2}{3}\left(\frac{x \lambda^{\prime}}{\lambda}\right)^{2}\right),(\log b)^{\prime} \simeq-\frac{1}{x}\left(1-\frac{2}{3} \frac{x \lambda^{\prime}}{\lambda}\right)
$$

The $\lambda$-dependent terms in the parentheses are $O(\lambda)$ or $O\left(\lambda^{2}\right)$.

One may naively think that it suffices to solve Schrödinger's equation keeping only the leading terms in (C.17), and neglecting the $O(\lambda)$ corrections. However, as we show below, these subleading terms affect the leading term in the solution.

Let us ignore for the moment the terms containing derivatives of $\lambda$ in equation (C.17). them, the near-boundary Schrödinger equation with the potential (C.5) reads:

$$
-\psi^{\prime \prime}+\frac{2}{x^{2}} \psi=\gamma^{2} \omega_{s}^{2} \psi
$$

whose general solution is:

$$
\psi_{\mathrm{UV}}^{0}(x)=A_{1}\left[\sin \left(\gamma \omega_{s} x\right)+\frac{\cos \left(\gamma \omega_{s} x\right)}{\gamma \omega_{s} x}\right]+A_{2}\left[\cos \left(\gamma \omega_{s} x\right)-\frac{\sin \left(\gamma \omega_{s} x\right)}{\gamma \omega x}\right]
$$


However, this cannot be the full story, for the following reason. For both small $x$ and small $\gamma \omega_{s} x$, we can expand this solution as:

$$
\psi_{\mathrm{UV}}^{0}(x) \simeq\left(\frac{A_{1}}{\gamma \omega_{s}}\right) \frac{1}{x}-\left(\frac{\gamma^{2} \omega_{s}^{2} A_{2}}{3}\right) x^{2}, \quad \gamma \omega_{s} x \ll 1
$$

On the other hand, in the same regime $\gamma \omega_{s} x \rightarrow 0$, we can ignore the constant term $\left(\gamma \omega_{s}\right)^{2}$ in the potential, and Scrhödinger equation becomes:

$$
\psi^{\prime \prime}=\frac{b^{\prime \prime}}{b} \psi
$$

which has the exact solution:

$$
\psi_{\mathrm{UV}}^{1}(x)=C_{s} b(x)+C_{v} b(x) \int_{0}^{x} \frac{d x^{\prime}}{b^{2}\left(x^{\prime}\right)}
$$

where $C_{s}$ and $C_{v}$ are integration constants corresponding to normalizable and nonnormalizable solutions. Using the near-boundary form of the metric (C.6) this expression becomes:

$$
\psi_{\mathrm{UV}}^{1}(x) \simeq \frac{C_{s}}{x} \lambda^{2 / 3}(x)+C_{v} x^{2} \lambda^{-2 / 3}(x), \quad \gamma \omega_{s} x \ll 1
$$

and it does not agree with the small $\gamma \omega_{s} x$ expansion (C.20) due to the extra factors of $\lambda^{ \pm 2 / 3}$. From this discussion, we conclude that to find the correct behavior of the solution near the boundary, one cannot completely ignore the terms containing $\lambda^{\prime}$ and $\lambda^{\prime \prime}$ in equation (C.17). Therefore, the true solution in the boundary region, rather than (C.19), will read instead:

$$
\begin{aligned}
\psi_{\mathrm{UV}}= & A_{1} \psi_{\text {source }}+A_{2} \psi_{\mathrm{vev}} \\
= & A_{1}\left[\sin \left(\gamma \omega_{s} x\right)+\frac{\cos \left(\gamma \omega_{s} x\right)}{\gamma \omega_{s} x}\right] F_{1}\left(x, \gamma \omega_{s}\right) \\
& +A_{2}\left[\cos \left(\gamma \omega_{s} x\right)-\frac{\sin \left(\gamma \omega_{s} x\right)}{\gamma \omega_{s} x}\right] F_{2}\left(x, \gamma \omega_{s}\right), \quad x \ll 1
\end{aligned}
$$

where $F_{1}$ and $F_{2}$ are some unknown functions, with asymptotics:

$$
F_{1}\left(x \gamma \omega_{s}\right) \sim \lambda^{2 / 3}(x), \quad F_{2}\left(x \gamma \omega_{s}\right) \sim \lambda^{-2 / 3}(x), \quad \gamma \omega_{s} x \ll 1 .
$$

On the other hand, we know that the functions $F_{1}$ and $F_{2}$ must be replaced by constants in the limit when $\lambda(x)$ is not changing at all. This suggests that we can parametrize the functions $F_{1}$ and $F_{2}$ as:

$$
F_{i}\left(x, \gamma \omega_{s}\right)=\lambda^{ \pm 2 / 3}(x)\left[1+\varphi_{i}\left(x, \gamma \omega_{s}\right)\right]
$$

where the functions $\varphi_{1,2}\left(x, \gamma \omega_{s}\right)$ are small compared to unity for small $x \lambda^{\prime} / \lambda$ and the $+\operatorname{sign}$ in the exponent of $\lambda$ corresponds to $i=1$, and the $-\operatorname{sign}$ to $i=2$.

One could in principle derive differential equations for $\varphi_{1,2}$, and solve them perturbatively. This is equivalent solving the fluctuation equation $h^{\prime \prime}+(\log R)^{\prime} h^{\prime}+\alpha h=0$ 
with some book-keeping parameter $\alpha$ and where the prime denotes derivative w.r.t. the variable $x \gamma \omega_{s}$. The solution can be found perturbatively in a series expansion both in $\alpha$ and $x$, such that $\lambda(x)$ can be regarded as a small expansion parameter. A simple limit of this solution is to keep only the leading term in the latter expansion and sum up the perturbative series in $\alpha$ fully and then set $\alpha=1$ to recover the original fluctuation equation. This can be achieved in an iterative manner and the answer is indeed given by (C.24) and (C.25). This method justifies the appearance of the extra factors $F_{1}$ and $F_{2}$ in (C.24).

However, proceeding with this method and combining it with the WKB approximation in order to achieve the full WKB solution requires going beyond the leading term in the perturbative series in the expansion in $\lambda(x)$, which is very cumbersome. Therefore, in the following we will follow a different strategy, which will give us a simpler (albeit more crude) way to estimate the coefficients of the WKB wave functions.

4. In the limit $\gamma \omega_{s} \gg 1$, the UV region $x \ll 1$ overlaps with the WKB region $x>x_{t p}$, because $x_{t p} \ll 1$. Therefore, the UV solution (C.24) must match, for large $\gamma \omega_{s}$, the small- $x$ limit of the WKB solution (C.13), which using the UV expansion of $R(x)$, equation (C.4), reads:

$$
\psi_{w k b} \simeq \frac{C_{1}}{\sqrt{\gamma}} \cos \left(\gamma \omega_{s} x\right)+\frac{C_{2}}{\sqrt{\gamma}} \sin \left(\gamma \omega_{s} x\right), \quad x \ll 1 .
$$

In order for (C.24) to agree with this expression, it is necessary that the functions $F_{1}(x)$ and $F_{2}(x)$ become approximately constant for $x \gg x_{t p}$, where (C.27) can be trusted. Since $x_{t p} \sim 1 / \omega_{s} \gamma$, we conclude that:

$$
F_{i}(x) \rightarrow F_{i}=\text { const } \quad \gamma \omega_{s} x \gg 1 .
$$

$i=1,2$. In order to complete the matching we must estimate these constants $F_{1}$ and $F_{2}$. One way to argue is as follows: to satisfy equation (C.28), we need the functions $\varphi_{1}$ and $\varphi_{2}$ defined in (C.26) to have the following property:

$$
\varphi_{i}(x) \rightarrow-1+\frac{F_{i}}{\lambda^{ \pm 2 / 3}(x)}, \quad x>x_{t p}
$$

again, where the + is for $i=1$, while the - for $i=2$. But as we argued that the functions $\varphi_{1}$ and $\varphi_{2}$ must stay small for slowly varying $\lambda$, setting $\varphi_{i} \approx 0$ gives us an estimate:

$$
F_{1} \simeq \lambda^{2 / 3}\left(x_{0}\right), \quad F_{2} \simeq \lambda^{-2 / 3}\left(x_{0}\right), \quad x \gg x_{t p}
$$

where $x_{0}$ is a point in the vicinity of the turning point $x_{t p}$. Since $\lambda$ is slowly varying, we can take $x_{0}=x_{t p}$, and the error we make will be of the order $x_{t p} \lambda^{\prime} / l \sim O(\lambda) \ll 1$.

Therefore, we match (C.27) with the large $\omega_{s}$ limit of (C.24), in which the functions $F_{1}$ and $F_{2}$ are replaced by the constants $\lambda^{ \pm 2 / 3}\left(x_{t p}\right)$ :

$$
\psi_{\mathrm{UV}} \rightarrow A_{1} \lambda_{t p}^{2 / 3} \sin \left(\gamma \omega_{s} x\right)+A_{2} \lambda_{t p}^{-2 / 3} \cos \left(\gamma \omega_{s} x\right)
$$


where we have defined $\lambda_{t p}=\lambda\left(x_{t p}\right)$. For large $\omega$ the turning point is given by:

$$
x_{t p} \simeq \frac{\sqrt{2}}{\omega_{s} \gamma}, \quad r_{t p} \simeq \frac{\sqrt{2}}{\omega \gamma} .
$$

Matching (C.31) and (C.27) we obtain:

$$
C_{1}=\lambda_{t p}^{-\frac{2}{3}} A_{2} \sqrt{\gamma}, \quad C_{2}=\lambda_{t p}^{\frac{2}{3}} A_{1} \sqrt{\gamma},
$$

which through equation (C.16) relates $C_{h}$ to $A_{1}$.

5. Finally, we determine $A_{1}$, and consequently all other coefficients, by imposing unit normalization of the function $\Psi(x)=\mathcal{R}^{-1 / 2} \psi(x)$ at $x=r_{b} / r_{s}$, i.e. the point where the string is attached. For a quark with infinite mass, $r_{b}=0$; for finite mass $r_{b}=r_{Q}$ defined in equation (4.3). Therefore, we must distinguish the following two situations:

- Infinite Quark Mass. Taking into account the definition (C.2) and the asymptotics (C.4) and (C.6), we impose:

$$
1=\Psi(0, \omega)=\frac{\sqrt{\gamma} r_{s}}{\ell} \lim _{x \rightarrow 0} \lambda^{-2 / 3} A_{1} \psi_{\text {source }}=\frac{A_{1}}{\sqrt{\gamma} \ell \omega},
$$

where in the last line we used the definition $\omega_{s}=\omega r_{s}$.

Using the chain of equations (C.34), (C.33) and (C.16) we can finally fix, in the large $\omega$ regime, the coefficient $C_{h}$ as:

$$
\left|C_{h}\right|=\ell \gamma\left(4 \pi T_{s} r_{s}\right)^{1 / 2} \lambda_{t p}^{2 / 3} \omega
$$

From this one obtains the coefficient $\Psi_{h}$ defined in (4.9) using the relation $\Psi(x)=\mathcal{R}^{-1 / 2} \psi(x)$ and (C.8). This finally yields (4.39).

- Finite Quark Mass. In an analogous way as for an infinitely massive quark, we use the form of the solution C.24 to write the normalization condition at the cutoff $r_{Q}$. However, the background scale factor $b(x)$ cannot be approximated by the expression in (C.6) at a generic cutoff $r_{Q}$, but we rather need to keep and evaluate the full $\mathcal{R}^{-1 / 2}(x) \sim b(x) / \sqrt{\gamma}$ entering the expression for $\Psi(x)$, without approximating it to $\Psi(x) \sim \sqrt{\gamma} x \lambda(x)^{-2 / 3}$. In fact, the necessary condition allowing to make use of this approximation is that $\lambda(x) \ll 1$. The normalization condition then reads:

$$
1=\Psi\left(r_{Q}, \omega\right)=\mathcal{R}^{-1 / 2}\left(x_{Q}\right)\left[A_{1} \psi_{\text {source }}\left(x_{Q}\right)+A_{2} \psi_{v e v}\left(x_{Q}\right)\right] .
$$

We now have to use the general form of the functions $F_{1}$ and $F_{2}$, and keep both the source and vev solutions, since at $r_{Q}$ the solution $\psi_{v e v}$ in not negligible. Hence, using the relations (C.16) and (C.33) yielding

$$
A_{2}=i \lambda_{t p}^{4 / 3} A_{1},
$$


the normalization condition determines $A_{1}$ as

$$
\begin{aligned}
A_{1}= & \gamma \omega_{s} x_{Q} \mathcal{R}^{1 / 2}\left(x_{Q}\right)\left[F_{1}\left(x_{Q}\right)\left(\cos \left(\gamma \omega_{s} x\right)+\gamma \omega_{s} x \sin \left(\gamma \omega_{s} x\right)\right)\right. \\
& \left.+i \lambda_{t p}^{4 / 3} F_{2}\left(x_{Q}\right)\left(\sin \left(\gamma \omega_{s} x\right)-\gamma \omega_{s} x \cos \left(\gamma \omega_{s} x\right)\right)\right]^{-1} .
\end{aligned}
$$

Moreover the asymptotics of $F_{1}$ and $F_{2}$ must be generalized w.r.t. (C.25), since we need to evaluate them at $x_{Q}$, which is not necessarily close enough to the boundary to allow us to use (C.25) - namely if $\lambda\left(x_{Q}\right)$ is not very small. In fact, one should keep the exact form of the solution at zero frequency (C.23), which reads

$$
\psi_{\mathrm{UV}}^{1}(x) \simeq C_{s} \sqrt{\gamma} \mathcal{R}^{1 / 2}(x)+\frac{C_{v}}{\sqrt{\gamma}} \mathcal{R}^{1 / 2}(x) \int^{x} \frac{d x^{\prime}}{\mathcal{R}\left(x^{\prime}\right)} .
$$

Comparing it to (C.24) one obtains the following behavior of function $F_{1}$ and $F_{2}$ :

$$
F_{1}(x) \sim \frac{r_{s}}{\ell} \sqrt{\gamma} x \mathcal{R}^{1 / 2}(x), F_{2}(x) \sim \frac{\ell}{r_{s}} \frac{\mathcal{R}^{1 / 2}(x)}{\sqrt{\gamma} x^{2}} \int^{x} \frac{d x^{\prime}}{\mathcal{R}\left(x^{\prime}\right)}, \quad \gamma \omega_{s} x \ll 1 .
$$

Consequently, one gets the value of the modulus square of the coefficient $C_{h}$ :

$$
\begin{aligned}
\left|C_{h}\right|^{2}= & 4 \pi \gamma^{2} T_{s} r_{s}^{3} \lambda_{t p}^{4 / 3} \frac{x_{Q}^{2} \mathcal{R}\left(x_{Q}\right)}{F_{1}\left(r_{Q}\right)^{2}}\left[1+\left(\gamma \omega_{s} x_{Q}\right)^{2}\right. \\
& \left.+\left(\lambda_{t p}^{8 / 3} \frac{F_{2}\left(r_{Q}\right)^{2}}{F_{1}\left(r_{Q}\right)^{2}}-1\right)\left(\sin \left(\gamma \omega_{s} x_{Q}\right)-\gamma \omega_{s} x_{Q} \cos \left(\gamma \omega_{s} x_{Q}\right)\right)^{2}\right]^{-1}
\end{aligned}
$$

As we explained previously in this appendix, $F_{1}$ and $F_{2}$ are approximated by (C.39) and (C.30), respectively for $\gamma \omega_{s} x \ll 1$ and $x \gg x_{t p} \simeq \sqrt{2} / \gamma \omega$. Therefore, substituting this asymptotics in $\left|C_{h}\right|^{2}$ and using equation (4.17), we arrive at the results of section 4.4.

Throughout the calculation we assumed that one can neglect the $\mathcal{O}(\lambda)$ terms compared to terms of order $\mathcal{O}(1)$. This criterion is indeed satisfied in the numerical examples we study in this paper. In the cases where this is not satisfied, or one needs better accuracy in the WKB approximation, than one should work out the sub-leading corrections.

\section{Correlators in $\mathcal{N}=4$}

In this appendix we would like to collect and derive some results on the imaginary part of the retarded correlator for the $\mathcal{N}=4$ theory. Some features of this quantity were discussed in [18] (see also [22]). More specifically, in [18] the symmetric correlator for $\mathcal{N}=4$ - related to $\operatorname{Im} G_{R}$ by equation (4.18) - is numerically computed and an analytic approximation is proposed. Here we show the numeric result for $\operatorname{Im} G_{R}$ and compare it to a linear plus cubic function. The advantage of considering $\operatorname{Im} G_{R}$ with respect to $G_{\text {sym }}$ is the possibility of distinguishing the corrections to the large and small frequency behavior 
associated to the coth factor in (4.18) from the wave function corrections, appearing in the coefficient $\Psi_{h}$ in (4.17). In $G_{\text {sym }}$ both kinds of corrections arise, while in $\operatorname{Im} G_{R}$ only the wave function contributes.

Unlike the non conformal case, the $\mathcal{N}=4$ correlators for the longitudinal and transverse modes only differ by a factor $\gamma^{2}$, due to the fact that the wave functions satisfy the same equation for both kinds of modes. The extra $\gamma^{2}$ comes from the $Z^{-2}$ factor in equation (4.1) for the longitudinal modes, that is constant in the conformal limit: $Z \rightarrow 1 / \gamma$. Moreover, the fluctuation equation for both transverse and longitudinal modes only depends on the dimensionless variables $x \equiv r / r_{s}$ and $\tilde{\omega} \equiv \frac{\omega}{4 \pi T_{s}}=\frac{\omega \sqrt{\gamma}}{4 \pi T}$ :

$$
\partial_{x}\left[\frac{1-x^{4}}{x^{2}} \partial_{x} \Psi(x, \tilde{\omega})\right]+\frac{(4 \tilde{\omega})^{2}}{x^{2}\left(1-x^{4}\right)} \Psi(x, \tilde{\omega})=0 .
$$

Following the same steps as in section 4, we obtain:

$$
\begin{aligned}
\Psi_{R}(\xi, \tilde{\omega}) & =C(\tilde{\omega})(1-x)^{-i \tilde{\omega}}+\cdots \\
\operatorname{Im} G_{R}^{\perp} & =\frac{2}{\pi} \sqrt{\lambda_{\mathcal{N}=4}} \sqrt{\gamma}(\pi T)^{3} \tilde{\omega}|C(\tilde{\omega})|^{2}
\end{aligned}
$$

The WKB approximation implies that the imaginary part of the retarded correlator grows as $\omega^{3}$ for large frequencies, in the infinite mass case. More precisely, taking the conformal limit, $b(r) \rightarrow \ell / r$ and $\ell / \ell_{s} \rightarrow \lambda_{\mathcal{N}=4}^{1 / 4}$, we obtain

$$
C(\tilde{\omega})=4 \tilde{\omega}+\mathcal{O}(1)
$$

and

$$
\operatorname{Im} G_{R}^{\perp}=\gamma^{-2} \operatorname{Im} G_{R}^{\|} \simeq \frac{\gamma^{2}}{2 \pi} \sqrt{\lambda_{\mathcal{N}=4}} \omega^{3}, \quad \text { for } \omega \gg \frac{1}{r_{s}} .
$$

This result ${ }^{18}$ can be obtained by applying the WKB method of the previous subsection the the wave functions obeying equation (D.1).

On the other hand, the result for small frequencies is well known [16, 18, 22] since it provides the diffusion constants. ${ }^{19}$ It is derived by analyzing the wave function solution to (D.1), in the regime where $\omega r_{s}=4 \tilde{\omega}$ is small. The expressions for small frequencies read

$$
C(\tilde{\omega})=1+\mathcal{O}(\tilde{\omega})
$$

and

$$
\operatorname{Im} G_{R}^{\perp}=\gamma^{-2} \operatorname{Im} G_{R}^{\|} \simeq \frac{\pi \gamma}{2} \sqrt{\lambda_{\mathcal{N}=4}} T^{2} \omega, \quad \text { for } \omega \ll \frac{1}{r_{s}} .
$$

This result can also follow from taking the conformal limit of the diffusion constants derived in the next section. In fact, equations (5.7)-(5.8) are related to (D.7) by the formula (5.1).

\footnotetext{
${ }^{18}$ The fact that the large- $\omega$ limit is a cubic power-law can be expected from the corresponding zerotemperature result, in which one can analytically compute this quantity, and from the consideration that the large-frequency limit should be conformal.

${ }^{19}$ In reference [22] it is claimed that the imaginary part of the correlator is exactly linear in $\omega$, for all frequencies. This is not so, as explicitly shown here.
} 

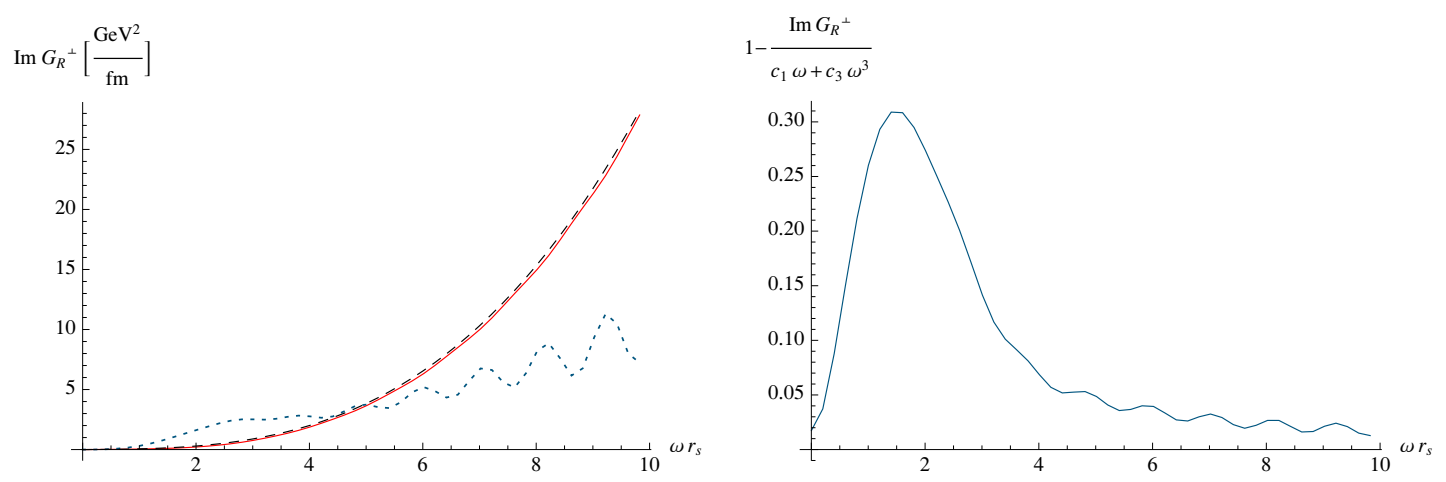

Figure 15. The picture on the left shows three curves (for $\lambda_{\mathcal{N}=4}=5.5$ and $T=250 \mathrm{MeV}$ ): i) the numeric result for $\operatorname{Im} G_{R}$ (red), ii) the linear plus cubic function $c_{1} \omega+c_{3} \omega^{3}$ where we use (D.9) for $c_{1}$ and $c_{3}$ (black dashed), iii) the difference of the linear plus cubic function with respect to the numeric result for $\operatorname{Im} G_{R}$, multiplied by a factor of 20 (blue dotted). The right plot shows the relative difference between the linear plus cubic function and the numeric result for the correlator.

In figure 15 we show the numeric result for the $\mathcal{N}=4$ correlator for the transverse modes compared to a linear plus cubic polynomial approximation,

$$
\operatorname{Im} G_{R}^{\perp} \approx c_{1} \omega+c_{3} \omega^{3}
$$

with $c_{1}$ and $c_{3}$ given by the small and large frequency asymptotics of the correlator, as in equations (D.7) and (D.5), respectively:

$$
c_{1}=\frac{\pi \gamma}{2} \sqrt{\lambda_{\mathcal{N}=4}} T^{2} \quad \text { and } \quad c_{3}=\frac{\gamma^{2}}{2 \pi} \sqrt{\lambda_{\mathcal{N}=4}}
$$

The relative difference between the polynomial (D.8) and the numeric result for the imaginary part of the retarded correlator vanishes, as expected, both for small and for large frequencies. Nevertheless, the plots of figure 15 show that for $1 \lesssim \omega r_{s} \lesssim 4$ there is a sensible difference, of the order of $10-30 \%$, between the two results. ${ }^{20}$

In the non-conformal cases the $\omega \rightarrow \infty$ limit of the correlator is again controlled by the UV. Conformal invariance again implies an $\mathcal{O}\left(\omega^{3}\right)$ behavior although it may be corrected by logarithms.

It is also interesting to study the sub-leading corrections to $\operatorname{Im} G_{R}$ in the freqency. We first consider small $\omega$. The leading term is determined by the Kubo's formula to be linear. On the other hand in any P-invariant quantum field theory the imaginary part of a retarded correlator is guaranteed to be odd in $\omega$, see for example [63] for a recent discussion. Therefore we learn that the sub-leading correction at small frequencies is cubic.

At high frequencies, the question is answered by extending the WKB analysis of appendix $\mathrm{C}$ to sub-leading frequencies. First of all we note that the form of the Schrodinger

\footnotetext{
${ }^{20}$ In figure 15 we had to fix a value for $T$ and $\lambda_{\mathcal{N}=4}$ in order to plot $\operatorname{Im} G_{R}$, but it is important to stress that the dependence on these quantities is simply given by the overall prefactor appearing in equation (D.3): the non-trivial part of the correlator depends on $T$ only through the combination $\tilde{\omega}=\omega / T_{s}$, and is independent of $\lambda_{\mathcal{N}=4}$.
} 
potential near horizon as written in (C.9). Now, the WKB wave-functions are obtained from this by making use of (C.12) and (C.13). The piece that is relevant for the current discussion is the $1 / \sqrt{p}$ part that in front of the cosine and the sine terms that clearly yields a $\mathcal{O}\left(\omega^{-2}\right.$ correction to the wave-functions in the large $\omega$ limit. Matching the WKB solution to the near-horizon solution as in appendix $\mathrm{C}$, yields $C_{2}=i C_{1}$ and one clearly obtains,

$$
C_{h} \propto C_{1}\left(1+\mathcal{O}\left(\omega^{-2}\right)\right)
$$

as a correction to equation (C.16).

On the other hand, the Schrodinger equation near the boundary, in the $\mathcal{N}=4$ theory, can be written as a function of $x \omega_{s}$. Therefore the wave-function near the boundary is a function of $x \omega_{s}$. This means that, upon demanding unit normalization of the source term - the coefficient $A_{1}$ in appendix C- one always has $A_{1} \times 1 / \omega \sim 1$ with no correction perturbative in $1 / \omega$. Therefore matching the wave-functions near the boundary and the WKB region yields $C_{1} \propto A_{1} \propto \omega$. Substituting in (D.10) we learn that $C_{h} \sim \omega+\mathcal{O}\left(\omega^{-1}\right)$. Finally, using (4.17) that determines the frequency dependence as $\operatorname{Im} G_{R} \propto \omega\left|C_{h}\right|^{2}$, we find that the subleading correction is linear in $\omega$, in the high $\omega$ limit.

Open Access. This article is distributed under the terms of the Creative Commons Attribution Noncommercial License which permits any noncommercial use, distribution, and reproduction in any medium, provided the original author(s) and source are credited.

\section{References}

[1] STAR collaboration, J. Adams et al., Experimental and theoretical challenges in the search for the quark gluon plasma: The STAR collaboration's critical assessment of the evidence from RHIC collisions, Nucl. Phys. A 757 (2005) 102 [nucl-ex/0501009] [SPIRES].

[2] B.B. Back et al., The PHOBOS perspective on discoveries at RHIC, Nucl. Phys. A 757 (2005) 28 [nucl-ex/0410022] [SPIRES].

[3] BRAHMS collaboration, I. Arsene et al., Quark Gluon Plasma an Color Glass Condensate at RHIC? The perspective from the BRAHMS experiment, Nucl. Phys. A 757 (2005) 1 [nucl-ex/0410020] [SPIRES].

[4] PHENIX collaboration, K. Adcox et al., Formation of dense partonic matter in relativistic nucleus nucleus collisions at RHIC: Experimental evaluation by the PHENIX collaboration, Nucl. Phys. A 757 (2005) 184 [nucl-ex/0410003] [SPIRES].

[5] D.T. Son and A.O. Starinets, Viscosity, Black Holes and Quantum Field Theory, Ann. Rev. Nucl. Part. Sci. 57 (2007) 95 [arXiv: 0704.0240] [SPIRES].

[6] J. Casalderrey-Solana and C.A. Salgado, Introductory lectures on jet quenching in heavy ion collisions, Acta Phys. Polon. B 38 (2007) 3731 [arXiv:0712.3443] [SPIRES].

[7] E. Iancu, Partons and jets in a strongly-coupled plasma from AdS/CFT, Acta Phys. Polon. B 39 (2008) 3213 [arXiv:0812.0500] [SPIRES].

[8] S.S. Gubser, S.S. Pufu, F.D. Rocha and A. Yarom, Energy loss in a strongly coupled thermal medium and the gauge-string duality, arXiv:0902.4041 [SPIRES]. 
[9] U. Gürsoy, E. Kiritsis, L. Mazzanti, G. Michalogiorgakis and F. Nitti, Improved Holographic $Q C D$, arXiv:1006.5461 [SPIRES].

[10] C.P. Herzog, A. Karch, P. Kovtun, C. Kozcaz and L.G. Yaffe, Energy loss of a heavy quark moving through $N=4$ supersymmetric Yang-Mills plasma, JHEP 07 (2006) 013 [hep-th/0605158] [SPIRES].

[11] H. Liu, K. Rajagopal and U.A. Wiedemann, Calculating the jet quenching parameter from AdS/CFT, Phys. Rev. Lett. 97 (2006) 182301 [hep-ph/0605178] [SPIRES].

[12] H. Liu, K. Rajagopal and U.A. Wiedemann, Wilson loops in heavy ion collisions and their calculation in AdS/CFT, JHEP 03 (2007) 066 [hep-ph/0612168] [SPIRES].

[13] S.S. Gubser, Drag force in AdS/CFT, Phys. Rev. D 74 (2006) 126005 [hep-th/0605182] [SPIRES].

[14] H. Liu, K. Rajagopal and Y. Shi, Robustness and Infrared Sensitivity of Various Observables in the Application of AdS/CFT to Heavy Ion Collisions, JHEP 08 (2008) 048 [arXiv:0803.3214] [SPIRES].

[15] U. Gürsoy, E. Kiritsis, G. Michalogiorgakis and F. Nitti, Thermal Transport and Drag Force in Improved Holographic QCD, JHEP 12 (2009) 056 [arXiv:0906.1890] [SPIRES].

[16] J. Casalderrey-Solana and D. Teaney, Heavy quark diffusion in strongly coupled $N=4$ Yang-Mills, Phys. Rev. D 74 (2006) 085012 [hep-ph/0605199] [SPIRES].

[17] C.P. Herzog and D.T. Son, Schwinger-Keldysh propagators from AdS/CFT correspondence, JHEP 03 (2003) 046 [hep-th/0212072] [SPIRES].

[18] S.S. Gubser, Momentum fluctuations of heavy quarks in the gauge-string duality, Nucl. Phys. B 790 (2008) 175 [hep-th/0612143] [SPIRES].

[19] J. Casalderrey-Solana and D. Teaney, Transverse momentum broadening of a fast quark in a $N=4$ Yang-Mills plasma, JHEP 04 (2007) 039 [hep-th/0701123] [SPIRES].

[20] J. de Boer, V.E. Hubeny, M. Rangamani and M. Shigemori, Brownian motion in AdS/CFT, JHEP 07 (2009) 094 [arXiv: 0812.5112] [SPIRES].

[21] D.T. Son and D. Teaney, Thermal Noise and Stochastic Strings in AdS/CFT, JHEP 07 (2009) 021 [arXiv:0901.2338] [SPIRES].

[22] G.C. Giecold, E. Iancu and A.H. Mueller, Stochastic trailing string and Langevin dynamics from AdS/CFT, JHEP 07 (2009) 033 [arXiv: 0903.1840] [SPIRES].

[23] E. Caceres, M. Chernicoff, A. Guijosa and J.F. Pedraza, Quantum Fluctuations and the Unruh Effect in Strongly-Coupled Conformal Field Theories, JHEP 06 (2010) 078 [arXiv: 1003.5332] [SPIRES].

[24] C. Hoyos-Badajoz, Drag and jet quenching of heavy quarks in a strongly coupled $N=2 *$ plasma, JHEP 09 (2009) 068 [arXiv:0907.5036] [SPIRES].

[25] PHENIX collaboration, Y. Akiba, Probing the properties of dense partonic matter at RHIC, Nucl. Phys. A 774 (2006) 403 [nucl-ex/0510008] [SPIRES].

[26] PHENIX collaboration, S.S. Adler et al., Nuclear modification of electron spectra and implications for heavy quark energy loss in Au + Au collisions at $s(N N)^{* *}(1 / 2)=200-G e V$, Phys. Rev. Lett. 96 (2006) 032301 [nucl-ex/0510047] [SPIRES]. 
[27] STAR collaboration, B.I. Abelev et al., Transverse momentum and centrality dependence of high-p $p_{T}$ non-photonic electron suppression in Au+Au collisions at $\sqrt{s_{N N}}=200 \mathrm{GeV}$, Phys. Rev. Lett. 98 (2007) 192301 [nucl-ex/0607012] [SPIRES].

[28] PHENIX collaboration, A. Adare et al., Energy Loss and Flow of Heavy Quarks in Au+Au Collisions at $\sqrt{s_{N N}}=200$ GeV, Phys. Rev. Lett. 98 (2007) 172301 [nucl-ex/0611018] [SPIRES].

[29] PHENIX collaboration, T.C. Awes, Highlights from PHENIX-II, J. Phys. G 35 (2008) 104007 [arXiv: 0805.1636] [SPIRES].

[30] N. Armesto, M. Cacciari, A. Dainese, C.A. Salgado and U.A. Wiedemann, How sensitive are high- $p_{T}$ electron spectra at RHIC to heavy quark energy loss?, Phys. Lett. B 637 (2006) 362 [hep-ph/0511257] [SPIRES].

[31] R. Rapp and H. van Hees, Heavy Quarks in the quark-gluon Plasma, arXiv:0903.1096 [SPIRES].

[32] Y. Akamatsu, T. Hatsuda and T. Hirano, Heavy Quark Diffusion with Relativistic Langevin Dynamics in the quark-gluon Fluid, Phys. Rev. C 79 (2009) 054907 [arXiv:0809.1499] [SPIRES].

[33] F. Debbasch, K. Mallick and J.P. Rivet, Relativistic Ornstein-Uhlenbeck process, J. Stat. Phys. 88 (1997) 945.

[34] F. Debbasch and J.P. Rivet, A diffusion equation from the relativistic Ornstein-Uhlenbeck process, J. Stat. Phys. 90 (1998) 1179.

[35] C. Chevalier and F. Debbasch, Relativistic diffusions: a unifying approach., J. Math. Phys. 49 (2008) 043303.

[36] J. Dunkel and P. Hänggi, Relativistic Brownian Motion, Phys. Rep. 471 (2009) 1 [arXiv: 0812.1996].

[37] S.S. Gubser, Comparing the drag force on heavy quarks in $N=4$ super-Yang-Mills theory and QCD, Phys. Rev. D 76 (2007) 126003 [hep-th/0611272] [SPIRES].

[38] U. Gürsoy and E. Kiritsis, Exploring improved holographic theories for QCD: Part I, JHEP 02 (2008) 032 [arXiv: 0707.1324] [SPIRES].

[39] U. Gürsoy, E. Kiritsis and F. Nitti, Exploring improved holographic theories for QCD: Part II, JHEP 02 (2008) 019 [arXiv: 0707.1349] [SPIRES].

[40] S.S. Gubser and A. Nellore, Mimicking the QCD equation of state with a dual black hole, Phys. Rev. D 78 (2008) 086007 [arXiv:0804.0434] [SPIRES].

[41] U. Gürsoy, E. Kiritsis, L. Mazzanti and F. Nitti, Deconfinement and Gluon Plasma Dynamics in Improved Holographic QCD, Phys. Rev. Lett. 101 (2008) 181601 [arXiv: 0804.0899] [SPIRES].

[42] U. Gürsoy, E. Kiritsis, L. Mazzanti and F. Nitti, Holography and Thermodynamics of $5 D$ Dilaton-gravity, JHEP 05 (2009) 033 [arXiv: 0812.0792] [SPIRES].

[43] E. Kiritsis, Dissecting the string theory dual of QCD, Fortsch. Phys. 57 (2009) 396 [arXiv: 0901.1772] [SPIRES].

[44] U. Gürsoy, E. Kiritsis, L. Mazzanti and F. Nitti, Improved Holographic Yang-Mills at Finite Temperature: Comparison with Data, Nucl. Phys. B 820 (2009) 148 [arXiv:0903.2859] [SPIRES]. 
[45] M. Panero, Thermodynamics of the QCD plasma and the large-N limit, Phys. Rev. Lett. 103 (2009) 232001 [arXiv:0907.3719] [SPIRES].

[46] E. Kiritsis, Supergravity, D-brane probes and thermal super Yang-Mills: A comparison, JHEP 10 (1999) 010 [hep-th/9906206] [SPIRES].

[47] A. Karch and A. O'Bannon, Metallic AdS/CFT, JHEP 09 (2007) 024 [arXiv:0705.3870] [SPIRES].

[48] J. Casalderrey-Solana, D. Fernandez and D. Mateos, A New Mechanism of Quark Energy Loss, Phys. Rev. Lett. 104 (2010) 172301 [arXiv:0912.3717] [SPIRES].

[49] L.F. Cugliandolo, J. Kurchan and L. Peliti, Energy flow, partial equilibration and effective temperatures in systems with slow dynamics, Phys. Rev. E 55 (1997) 3898 [SPIRES].

[50] D. Cubero, J. Casado-Pascual, J. Dunkel, P. Talkner and P. Hänggi, Thermal equilibrium and statistical thermometers in special relativity, Phys. Rev. Lett. 99 (2007) 170601 [arXiv: 0705.3328].

[51] N. Iqbal and H. Liu, Universality of the hydrodynamic limit in AdS/CFT and the membrane paradigm, Phys. Rev. D 79 (2009) 025023 [arXiv:0809.3808] [SPIRES].

[52] D. Teaney, Finite temperature spectral densities of momentum and $R$ - charge correlators in $N=4$ Yang-Mills theory, Phys. Rev. D 74 (2006) 045025 [hep-ph/0602044] [SPIRES].

[53] J. Casalderrey-Solana and C.A. Salgado, Introductory lectures on jet-quenching in heavy ion collisions, Acta Phys. Polon. B 38 (2007) 3731 [arXiv: 0712.3443] [SPIRES].

[54] R.P. Feynman and F.L. Vernon, The theory of a general quantum system interacting with a linear dissipative system, Annals Phys. 24 (1963) 118 [Annals Phys. 281 (2000) 547] [SPIRES].

[55] H. Kleinert, PATH INTEGRALS in Quantum Mechanics, Statistics, Polymer Physics, and Financial Markets, World Scientific, Singapore (2004) [SPIRES].

[56] S.S. Gubser, Comparing the drag force on heavy quarks in $N=4$ super-Yang-Mills theory and QCD, Phys. Rev. D 76 (2007) 126003 [hep-th/0611272] [SPIRES].

[57] D.T. Son and A.O. Starinets, Minkowski-space correlators in AdS/CFT correspondence: Recipe and applications, JHEP 09 (2002) 042 [hep-th/0205051] [SPIRES].

[58] S.S. Gubser, S.S. Pufu and F.D. Rocha, Bulk viscosity of strongly coupled plasmas with holographic duals, JHEP 08 (2008) 085 [arXiv:0806.0407] [SPIRES].

[59] K.B. Fadafan, H. Liu, K. Rajagopal and U.A. Wiedemann, Stirring Strongly Coupled Plasma, Eur. Phys. J. C 61 (2009) 553 [arXiv:0809.2869] [SPIRES].

[60] F. Bigazzi et al., D3-D7 quark-gluon Plasmas, JHEP 11 (2009) 117 [arXiv:0909.2865] [SPIRES].

[61] C. Núñez, A. Paredes and A.V. Ramallo, Unquenched flavor in the gauge/gravity correspondence, Adv. High Energy Phys. 2010 (2010) 196714 [arXiv:1002.1088] [SPIRES].

[62] G. Bertoldi, F. Bigazzi, A.L. Cotrone and J.D. Edelstein, Holography and Unquenched Quark-Gluon Plasmas, Phys. Rev. D 76 (2007) 065007 [hep-th/0702225] [SPIRES].

[63] F. Karsch, D. Kharzeev and K. Tuchin, Universal properties of bulk viscosity near the QCD phase transition, Phys. Lett. B 663 (2008) 217 [arXiv:0711.0914] [SPIRES]. 